\title{
THE TOPOLOGY AND ANALYSIS OF THE HANNA NEUMANN CONJECTURE
}

\author{
IGOR MINEYEV
}

Abstract. The statement of the Hanna Neumann Conjecture (HNC) is purely algebraic: for a free group $\Gamma$ and any nontrivial finitely generated subgroups $A$ and $B$ of $\Gamma$,

$$
\operatorname{rk}(A \cap B)-1 \leq(\operatorname{rk} A-1)(\operatorname{rk} B-1) .
$$

The goal of this paper is to systematically develop machinery that would allow for generalizations of $\mathrm{HNC}$ and to exhibit their relations with topology and analysis. On the topological side we define immersions of complexes, leafages, systems of complexes, flowers, gardens, and atomic decompositions of graphs and surfaces. The analytic part involves working with the classical Murray-von Neumann (!) dimension of Hilbert modules.

This also gives an approach to the Strengthened Hanna Neumann Conjecture (SHNC) and to its generalizations. We present three faces of it named, respectively, the square approach, the diagonal approach, and the arrangement approach. Each of the three comes from the notion of a system, and each leads to questions beyond graphs and free groups. Partial results, sufficient conditions, and generalizations of the statement of SHNC are presented.

\section{Contents}

1. Introduction

2. Groups and complexes.

3. Systems. 6

4. Groups and Hilbert modules. 16

5. Graphs. 21

6. Change of subgroups. 27

7. Restatements of SHNC. 31

8. Surfaces.

9. The diagonal approach. 38

10. The square approach.

11. Subspaces and arrangements. $\quad 54$

12. Ergodicity. $\quad 57$

13. Multiplicativity. $\quad 61$

14. Questions.

References $\quad 63$

Date: April 30, 2010. Last revision May 21, 2011. 


\begin{abstract}
Although the summer sunlight gild
Cloudy leafage of the sky,

Or wintry moonlight sink the field

In storm-scattered intricacy,

I cannot look thereon,

Responsibility so weighs me down.
\end{abstract}

William Butler Yeats, "Vacillation".

\title{
1. INTRODUCTION
}

Hanna Neumann formulated her conjecture in [17] and [18]. The easiest way to state it is using the notion of reduced rank defined by Walter Neumann [19, p. 162] and named so by Dicks [3, p. 373]. The reduced rank of a free group $\Gamma$ is the number

$$
\bar{r}(\Gamma):=\max \{0, \operatorname{rk} \Gamma-1\} .
$$

Conjecture 1 (HNC). Suppose $\Gamma$ is a free group and $A$ and $B$ are its finitely generated subgroups. Then

$$
\bar{r}(A \cap B) \leq \bar{r}(A) \cdot \bar{r}(B) .
$$

This conjecture, if true, would be the sharp version of the result of Howson [8] asserting that $\operatorname{rk}(A \cap B)$ is finite. Walter Neumann [19, p.164] further proposed the following Strengthened Hanna Neumann Conjecture. Let $A \backslash \Gamma / B$ be the set of all double cosets $A g B$ for $g \in \Gamma$ and $s: A \backslash \Gamma / B \rightarrow \Gamma$ be a section of the quotient map $\Gamma \rightarrow A \backslash \Gamma / B$. Denote $A^{z}:=z^{-1} A z$.

Conjecture 2 (SHNC). Suppose $\Gamma$ is a free group and $A$ and $B$ are its finitely generated subgroups. Then

$$
\sum_{z \in s(A \backslash \Gamma / B)} \bar{r}\left(A^{z} \cap B\right) \leq \bar{r}(A) \cdot \bar{r}(B) .
$$

A series of partial results have appeared since. For further details the reader is referred to Burns [2], Imrich [9], Servatius [23], Stallings [24], Gersten [7], Nickolas [20], Walter Neumann [19], Feuerman [6], Tardos [25], Dicks [3], Dicks-Formanek [4], Khan [13], MeakinWeil [16], Sergei Ivanov [10, 11], Dicks-Ivanov [5].

There is no loss of generality in assuming in either conjecture that $\Gamma$ is the free group of rank 2 , since the group generated by $A$ and $B$ can be embedded in $F_{2}$ (see [19]). The relation of HNC to a study of pull-backs of finite graphs goes back to the work of Stallings [24]; he showed that the subgroups $A$ and $B$ can be represented by immersions of finite graphs, and that $A \cap B$ is realized by a component of their fiber-product. It was first observed by Warren Dicks that HNC can be restated in terms of the first $\ell^{2}$-Betti numbers of $A, B$, and $A \cap B$.

Our goal is to set a foundation for HNC-like questions, generalizing the conjecture to complexes and arbitrary groups. Below is an outline of notions and results of the paper.

- Let $\Gamma$ be an arbitrary group, $\hat{X}$ be a free $\Gamma$-complex, $X:=\Gamma \backslash \hat{X}$, and $A, B \leq \Gamma$. In 3.1 we define a system of complexes and maps that arise from these data. 
- The case when $\Gamma$ is free can be realized by a system of graphs which incorporates Stallings' immersions (see 3.6 and 3.7). We show that in this case the reduced ranks of $\Gamma, A, B$ equals the first $\ell^{2}$-Betti numbers of $\Gamma$-graphs in a corresponding graph system (5.4 and 8.3), so the inequality of SHNC can be equivalently rewritten in terms of $\ell^{2}$ Betti numbers for that system. Then we work with Hilbert modules arising from such graphs systems.

- In particular, systems allow generalizing the statements of HNC and SHNC to surfaces: those are inequalities involving $\ell^{2}$-Betti numbers for finitely generated subgroups of $\pi_{1}(X)$, where $X$ is a compact surface (with boundary or not) with $\chi(X)=-1$ (section 8). This surface case turns out to be very similar to the case of graphs.

- Flowers and gardens in 5.1 are combinatorial notions parallel to trees and forests. They serve the same purpose for reduced rank as trees and forests do for the usual rank of free groups. Flowers and gardens describe $\ell^{2}$-Betti numbers just as trees and forests describe the usual Betti numbers of graphs.

- Atomic decompositions of graphs are defined in 5.5 and are used in 10.10 to obtain certain bases in Hilbert modules and atomic square maps. In a completely analogous fashion we describe atomic decompositions of surfaces in 8.4. Atoms naturally provide subspaces of dimension 1 (theorems 16 and 26).

- Tardos [25] proved SHNC in the case when one of the subgroups is of rank 2. We give alternative analytic proofs of this result and generalize it to surfaces (Corollary 35, Theorem 66). An analogous result can be proved for complexes when $\Gamma$ is any group satisfying the Atiyah conjecture.

- In 10.9 we define two classes of groups $\mathcal{P} \subseteq \mathcal{P}_{\text {lim }}$ in terms of bases in $\ell^{2}(\Gamma \times \Gamma)$, and ask, what groups they contain. Groups in these classes (at least those that satisfy the Atiyah conjecture) can be shown to satisfy SHNC-like inequalities. If $F_{2}$ belongs to $\mathcal{P}_{\text {lim }}$, then SHNC holds.

- It is well known that the equality holds in the statement of SHNC if one of the groups is of finite index (see [19]). We give alternative proofs of this and of its analog for surfaces (lemmas 71 and 72 ).

- We prove SHNC in the case when one of the graphs is "one edge less that a cover" (subsection 12.4); this can be viewed as dual to Tardos' result.

- In 11.5 we establish a kind of duality that allows equivalently restating the inequality in SHNC as an inequality for orthogonal complements.

- Sufficient conditions for SHNC are given in theorems 32, 51, 52, 56, 65, 67, and lemmas 58 and 74 .

- In section 12 we discuss a notion of "finite-dimensional" ergodicity for subspaces that naturally arises from the arrangement approach and seems to be relevant for SHNC.

- In section 13 we investigate multiplicativity, that is when an equality, rather than an inequality, holds in the statement of SHNC.

- Section 14 poses some questions. 
The following are the three faces of our approach to SHNC and to its generalizations. They all arise from the notion of a system, and all three lead to questions beyond graphs and free groups.

(1) Let the three graphs $(\hat{S}, \hat{Y}, \hat{Z})$ be part of the graph system introduced in 3.6; they correspond to the three terms of inequality (2). The square approach (section 10) studies certain $G \times G$-equivariant maps $H_{1}^{(2)}(\hat{\hat{S}}) \rightarrow H_{1}^{(2)}(\hat{Y}) \hat{\otimes} H_{1}^{(2)}(\hat{Z})$, where $G$ is a subgroup of finite index in $\Gamma$. This can also be restated in terms of some particular $G \times G$-maps $\ell^{2}(\Gamma \times \Gamma) \rightarrow \ell^{2}(\Gamma \times \Gamma)$, that are obtained by "gliding the identity map along diagonals". All such maps are generically called square maps. The existence of an injective square map would imply SHNC. More generally, having a sequence $G_{i} \leq \Gamma$ and a sequence of square maps that "become more and more injective" would suffice. (See remark in 10.8.) Specific examples of square maps are obtained from systems (see 10.3, 10.5, 10.10) and we ask whether any of them are injective.

(2) The diagonal approach (section 9) uses only "the diagonal part" of the above tensor product. We give sufficient conditions for SHNC in terms of existence of certain $\Gamma$ invariant bases.

(3) The arrangement approach of section 11 is a restatement of the diagonal approach in terms of finite indexed families of Hilbert $G$-submodules in $\ell^{2}(\Gamma)$, called arrangements, where $G$ is a certain normal subgroup of finite index in $\Gamma$. SHNC is restated as a question about such arrangements. As a test case, one can ask the same question about arrangements of subspaces in a finite dimensional vector space that are invariant under some finite group.

The author would like to thank Victor Gerasimov, Stephen Gersten, Peter Linnell, Wolfgang Lück, Walter Neumann, Roman Sauer, Guoliang Yu for helpful conversations on related subjects. This research is partially supported by the NSF grant DMS 07-06876.

\section{Groups AND COMPLEXES.}

2.1. Cell complexes. For concrete applications to the Hanna Neumann conjecture we primarily will be interested in graphs and free groups, but it seems important to discuss cell complexes and more general groups since one of our objectives will be to explore generalizations of the conjecture. Even considering free groups, representing them as fundamental groups of surfaces or complexes conceivably might be beneficial.

We will work in the category of CW complexes, which will be called cell complexes. Let $\Sigma_{i}(X)$ denote the set of all closed $i$-cells in $X$ and $\Sigma(X):=\bigsqcup_{i \geq 0} \Sigma_{i}(X)$. The cells in a cell complex are assumed to be given fixed orientations. In the combinatorial language, each complex $X$ is viewed as the set $\Sigma(X)$. All maps of cell complexes and group actions are required to preserve cell structure and the orientations of cells.

$\operatorname{Comp}(Y)$ will denote the set of connected components of $Y$. 
The pull-back diagram

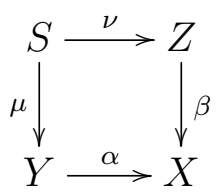

of cell maps $\alpha$ and $\beta$ is defined as follows. The fiber product $S$ of $Y$ and $Z$ is the set

$$
Y \times_{X} Z:=\{(y, z) \in Y \times Z \mid \alpha(y)=\beta(z)\}
$$

and $\mu$ and $\nu$ are projections to each coordinate.

2.2. Combinatorial maps. A map $\alpha: Y \rightarrow X$ between cell complexes will be called combinatorial if it maps each open cell in $X$ homeomorphically onto an open cell in $Y$. If $\alpha: Y \rightarrow X$ and $\beta: Z \rightarrow X$ are combinatorial maps, then $Y \times_{X} Z$ has a natural induced structure of cell complex such that $\Sigma\left(Y \times_{X} Z\right)$ can be identified with $\Sigma(Y) \times_{\Sigma(X)} \Sigma(Z)$. In other words, the topological and combinatorial pull-back operations agree, so we can use them interchangeably.

\subsection{Immersions.}

Definition 1. A continuous map $f: Y \rightarrow X$ between two cell complexes will be called an immersion if there exists a cover $f^{\prime}: Y^{\prime} \rightarrow X$, with cellular structure on $Y^{\prime}$ induced from $X$, such that $Y$ is a subcomplex of $Y^{\prime}$ and $f^{\prime}$ is an extension of $f$.

This generalizes immersions of graphs considered by Stallings [24]. Any immersion of finite graphs $Y \rightarrow X$ in the sense of [24] (restated for the category of 1-dimensional cell complexes rather than for Serre graphs) is an immersion in the sense of the above definition.

Each cover is obviously an immersion, and all immersions are combinatorial.

2.4. Cloudy leafage of the sky. A leafage will be a combinatorial map $\lambda: \hat{Y} \rightarrow \hat{X}$ between cell complexes whose restriction to each connected component of $\hat{Y}$ is injective. For brevity we will say in this case that " $\hat{Y}$ is a leafage over $\hat{X}$ " or that " $\lambda$ is a leafage over $\hat{X}$ ". A cover leafage is a leafage $\lambda: \hat{Y} \rightarrow \hat{X}$ that sends each connected component of $\hat{X}$ (isomorphically) onto a connected component of $\hat{Y}$. A $\Gamma$-leafage is a leafage $\lambda: \hat{Y} \rightarrow \hat{X}$ such that $\hat{X}$ and $\hat{Y}$ are given cellular $\Gamma$-actions and $\lambda$ is $\Gamma$-eqivariant.

The following lemma is immediate.

Lemma 2. Suppose

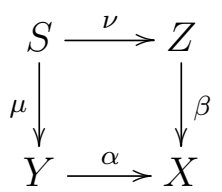

is any pull-back diagram of cell complexes.

(a) If $\alpha$ is an immersion and $\beta$ is a cover, then $\mu$ is a cover.

(b) If $\alpha$ and $\beta$ are cover, then $\mu, \nu$, and $\alpha \circ \mu$ are covers.

(c) If $\alpha$ and $\beta$ are immersions, then $\mu, \nu$, and $\alpha \circ \mu$ are immersions.

(d) If $\alpha$ is a leafage, then $\nu$ is a leafage. 


\section{Systems.}

3.1. Systems of complexes. We define a formal structure consisting of cell complexes and maps. It can be viewed as a category if needed.

Start with a group $\Gamma$ and a cell complex $\hat{X}$ with a free cellular $\Gamma$-action. For simplicity, in this paper we will always additionally assume that $\hat{X}$ is connected. This assumption is really a technical one and can be removed. Let $X:=\Gamma \backslash \hat{X}, p_{X}: \hat{X} \rightarrow X$ be the quotient map, and $\alpha: Y \rightarrow X$ and $\beta: Z \rightarrow X$ be immersions.

Notation. In what follows, for fixed complexes $X$ and $\hat{X}$,

- $Y \square Z$ will denote the fiber product $Y \times_{X} Z$, and

- $Y \hat{\square} Z$ will denote the fiber product $Y \times_{\hat{X}} Z$.

Define complexes $\hat{Y}, \hat{Z}, \hat{S}$ and the maps $p_{Y}, p_{Z}, p_{S}, \hat{\alpha}, \hat{\beta}, \hat{\mu}, \hat{\nu}$ by pulling back diagram (3) by $p_{X}$ :

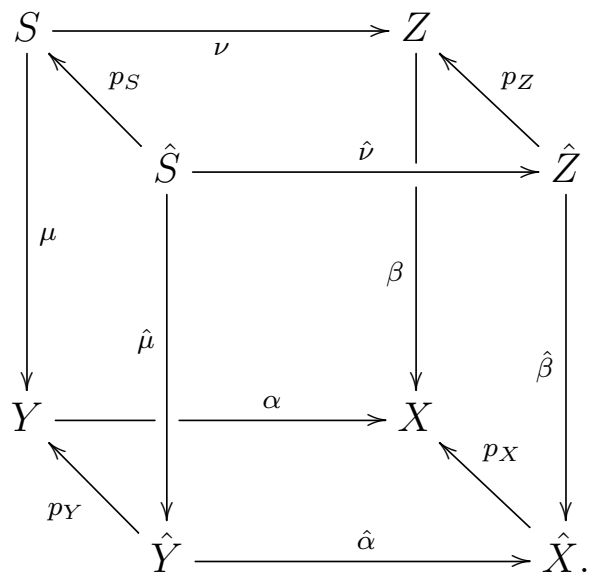

Each square in (4) is a pull-back diagram. In particular, we can identify $\hat{S}=\hat{Y} \hat{\square} \hat{Z}$. Define $\hat{S}$ by the pullback diagram

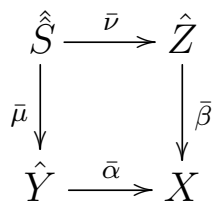


Then $\hat{S}=\hat{Y} \hat{\square} \hat{Z} \subseteq \hat{Y} \square \hat{Z}=\hat{\hat{S}}$. We let $\bar{\iota}: \hat{S} \rightarrow \hat{S}$ denote the inclusion map. The above square can be incorporated into (4) giving

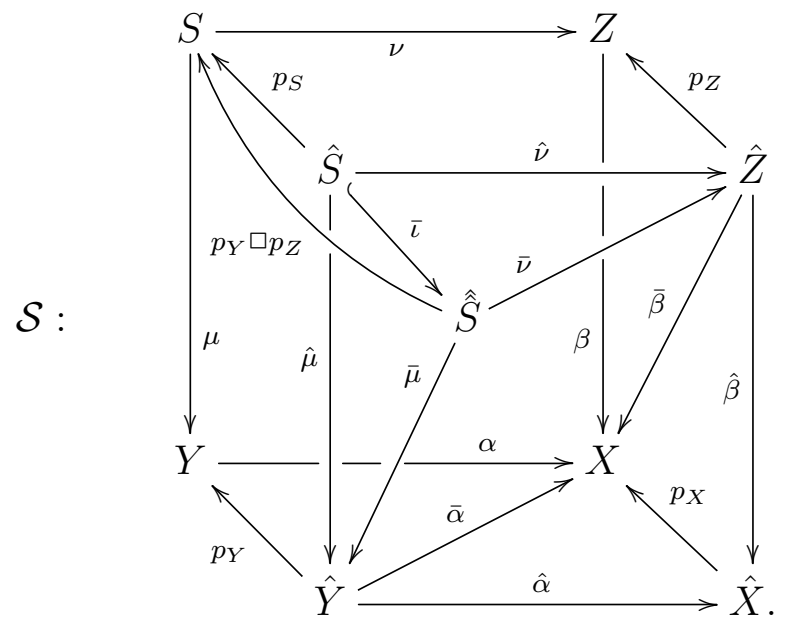

Diagrams (4) and (5) determine each other. Either one of them, together with the $\Gamma$-action on $\hat{X}$, will be called the system generated by $\left(X, Y, Z, \hat{X}, \alpha, \beta, p_{X}\right)$ and denoted

$$
\mathcal{S}=\mathcal{S}\left(X, Y, Z, \hat{X}, \alpha, \beta, p_{X}\right)
$$

Definition 3. A system, or a $\Gamma$-system, is the system generated by some $\left(X, Y, Z, \hat{X}, \alpha, \beta, p_{X}\right)$ as above. A system $\mathcal{S}$ will be called connected if $X, Y$ and $Z$ are connected.

3.2. A more detailed description of a system. Each commutative diagram can be viewed as a category whose objects are the entries and morphisms are the arrows together with the added identity arrows at each entry.

Consider the special case of system (4) in which $\alpha: Y \rightarrow X$ and $\beta: Z \rightarrow X$ are both taken to be the identity map $i d: X \rightarrow X$. This defines the system

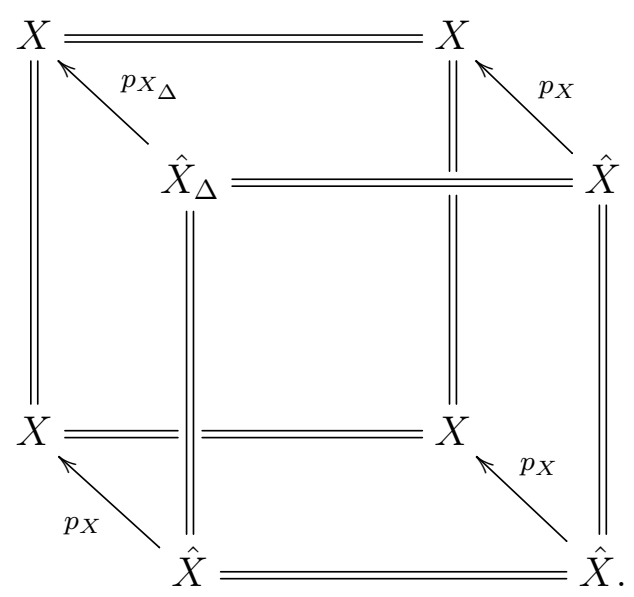


in which all the squares are pull-back diagrams. Clearly, $\hat{X}_{\Delta}$ is isomorphic to $\hat{X}$ and we can identify $\hat{X}_{\Delta}=\hat{X} \hat{\square} \hat{X}$.

Let $p_{X}: \hat{X} \rightarrow X$ be as above. Define $\hat{X}$ and $q_{X}$ by the pull-back diagram

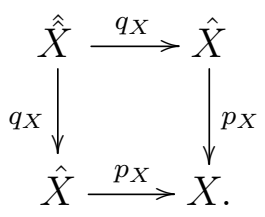

By definition, $\hat{X} \hat{\square} \hat{X} \subseteq \hat{X} \square \hat{X}$. Define the cellular map $\iota: \hat{X}_{\Delta} \rightarrow \hat{X}$ to be the inclusion

$$
\hat{X}_{\Delta}=\hat{X} \hat{\square} \hat{X} \subseteq \hat{X} \square \hat{X}=\hat{X}
$$

The above spaces and maps form the commutative diagram

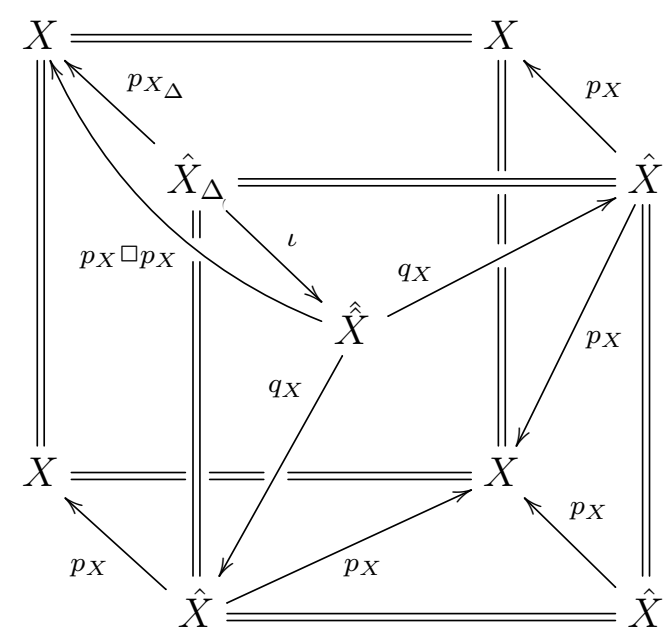

which is a special case of (5).

Extend the identity functor
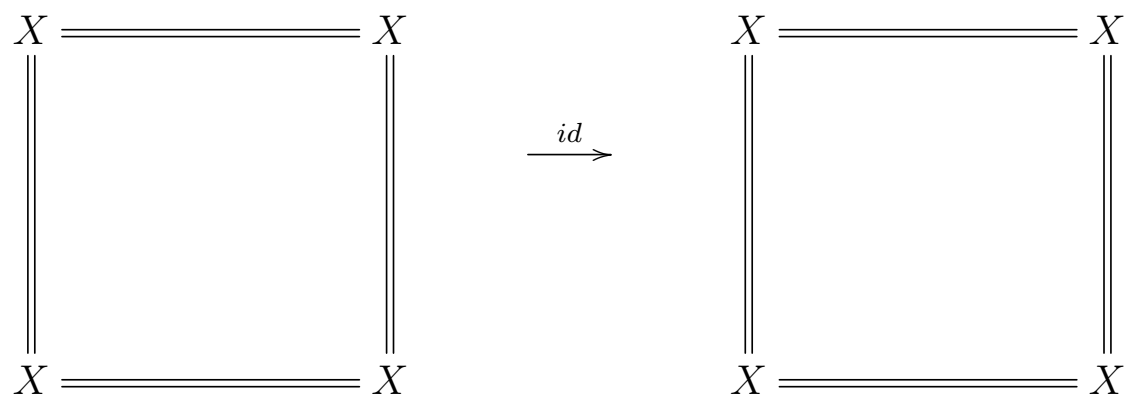
to the functor

(9)
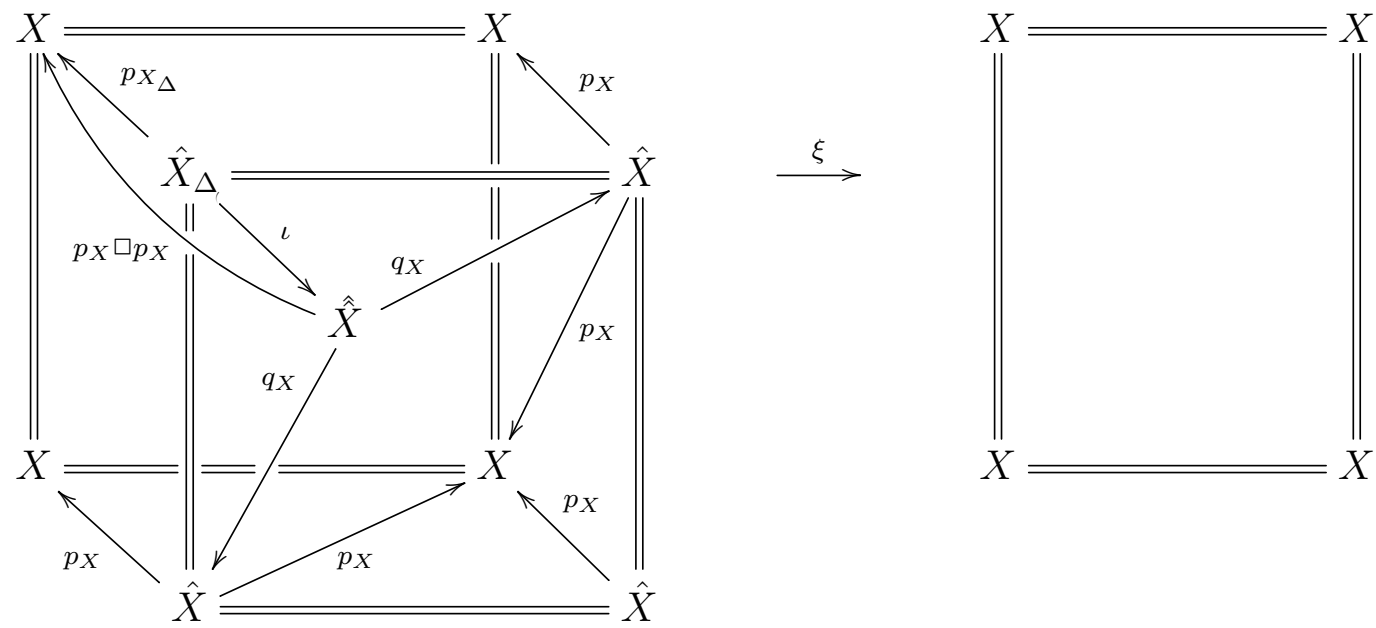

in the obvious way, by mapping each entry on the left to the corresponding corner of the square on the right by an appropriate map: $i d_{X}, p_{X}, p_{X_{\Delta}}$, and $p_{X} \square p_{X}$.

Now one can check that diagram (5) is the pull-back diagram of the functors $\theta$ and $\xi$, where $\theta$ is defined in the obvious way by maps into $X$. 
(10)

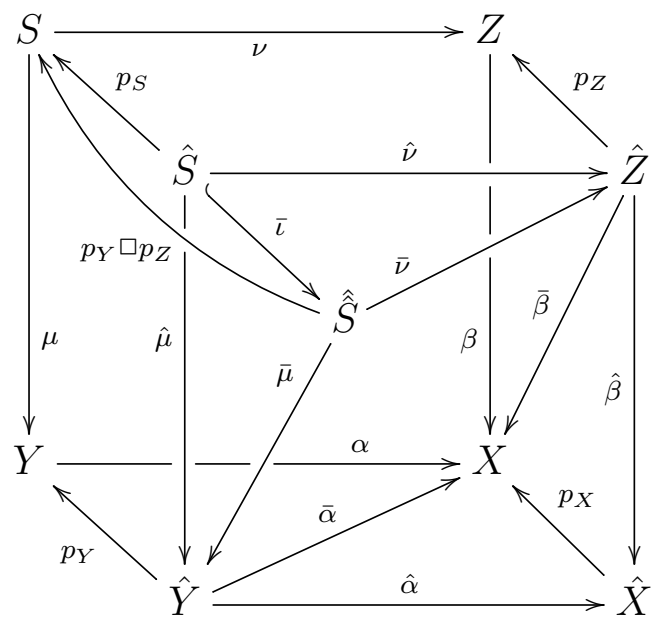

$\Theta \downarrow$

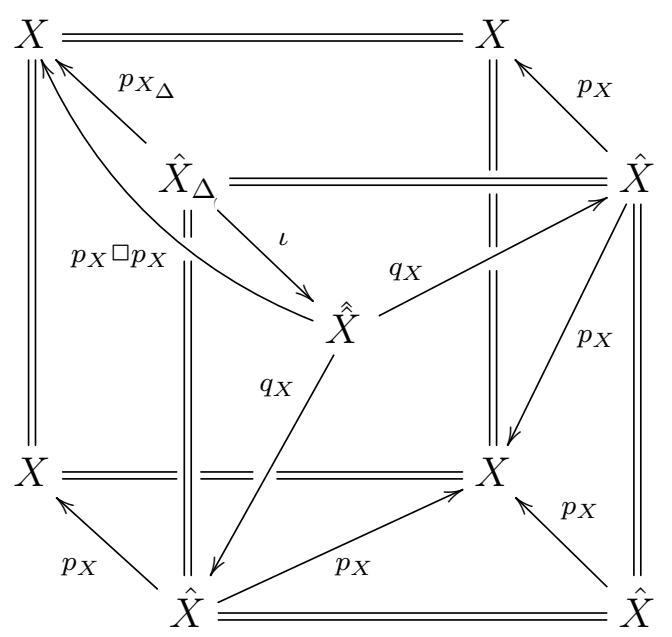

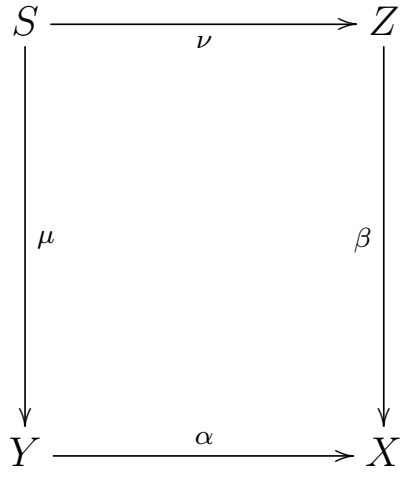
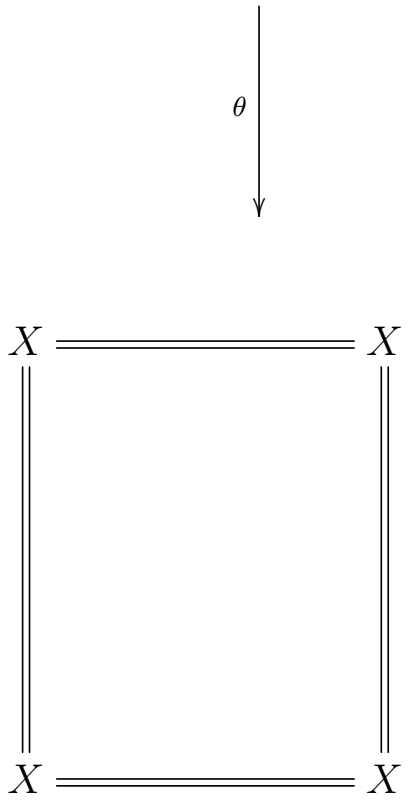

This pull-back defines each entry and each arrow in the system (5) which is the left upper corner of (10).

3.3. Group actions in the system. The $\Gamma$-action on $\hat{X}$ induces $\Gamma$-actions on $\hat{Y}$ and $\hat{Z}$. The $\Gamma$-actions on $\hat{X}$ and $\hat{Y}$ induce a coordinate-wise $\Gamma \times \Gamma$-action on $\hat{S}$ :

$$
(g, h) \cdot(\sigma, \tau):=(g \sigma, h \tau) \quad \text { for } \quad g, h \in \Gamma,(\tau, \sigma) \in \Sigma(\hat{S})=\Sigma(\hat{Y} \square \hat{Z}) .
$$


A similar formula defines a $\Gamma \times \Gamma$-action on $\hat{X}$.

Let $\Delta$ be the diagonal subgroup in $\Gamma \times \Gamma$.

\section{Lemma 4.}

(a) $\operatorname{Stab}_{\Gamma \times \Gamma}\left(\hat{X}_{\Delta}\right)=\Delta$.

(b) $\operatorname{Stab}_{\Gamma \times \Gamma}(\hat{S})=\Delta$.

Proof. (a)

$$
\Sigma\left(\hat{X}_{\Delta}\right)=\Sigma(\hat{X} \hat{\square} \hat{X})=\{(\sigma, \sigma) \in \Sigma(\hat{X}) \times \Sigma(\hat{X}) \mid \sigma \in \Sigma(\hat{X})\} \subseteq \Sigma(\hat{X}),
$$

so $\hat{X}_{\Delta}$ is the same as the image of the diagonal inclusion diag $: \hat{X} \hookrightarrow \hat{X} \times \hat{X}, \sigma \mapsto(\sigma, \sigma)$. This implies $\Delta \subseteq \operatorname{Stab}_{\Gamma \times \Gamma}\left(\hat{X}_{\Delta}\right)$. Conversely, take any $(g, h) \in \operatorname{Stab}_{\Gamma \times \Gamma}\left(\hat{X}_{\Delta}\right)$ and any $(\sigma, \sigma) \in \Sigma\left(\hat{X}_{\Delta}\right)$, then $(g \sigma, h \sigma) \in \Sigma\left(\hat{X}_{\Delta}\right)$, so $g \sigma=h \sigma$. Since the $\Gamma$-action on $\hat{X}$ is free, $g=h$ and $(g, h) \in \Delta$.

(b) $\hat{S}$ is the full preimage (= pull-back) of $\hat{X}_{\Delta}$ under the $\Gamma \times \Gamma$-map $\hat{S} \rightarrow \hat{X}$ from diagram (10), hence $\operatorname{Stab}_{\Gamma \times \Gamma}(\hat{S})=\operatorname{Stab}_{\Gamma \times \Gamma}\left(\hat{X}_{\Delta}\right)=\Delta$.

Therefore each term in the system $\mathcal{S}$ naturally comes with a group action:

- 1 acts on $X, Y$, and $Z$,

- $\Gamma$ acts on $\hat{X}, \hat{Y}$, and $\hat{Z}$,

- $\Gamma \times \Gamma$ acts on $\hat{S}$,

- $\Delta$ acts on $\hat{S}$.

$\hat{S}$ can be given a $\Gamma$-action by the diagonal isomorphism $\Gamma \rightarrow \Delta$. For the purpose of notation we will not distinguish between the $\Gamma$-action and the $\Delta$-action on $\hat{S}$, and will use them interchangeably.

Lemma 5. The maps $p_{X}, p_{Y}, p_{Z}$, and $p_{S}$ are the quotient maps by the $\Gamma$-actions. The map $p_{Y} \square p_{Z}$ is the quotient map by the $\Gamma \times \Gamma$-action. This gives the isomorphisms

$$
X \cong \Gamma \backslash \hat{X}, \quad Y \cong \Gamma \backslash \hat{Y}, \quad Z \cong \Gamma \backslash \hat{Z}, \quad S \cong \Gamma \backslash \hat{S}, \quad S \cong(\Gamma \times \Gamma) \backslash \hat{S} .
$$

Proof. $p_{X}$ is the quotient map meaning that the preimage of each cell in $X$ under the map $\tilde{X} \rightarrow X$ is a $\Gamma$-orbit. This property lifts to the maps $\hat{Y} \rightarrow Y, \hat{Z} \rightarrow Z, \hat{S} \rightarrow S$. The $\Gamma \times \Gamma$-orbits of cells in $\hat{S}$ are exactly the Cartesian products of $\Gamma$-orbits in $\hat{Y}$ and $\hat{Z}$ mapping to the same cell in $X$, and those are exactly the preimages in $\hat{S}$ of cells in $X$.

3.4. Subsystems and pairs. Let $\mathcal{S}$ be any system. A subsystem of a system $\mathcal{S}$ has the obvious meaning: it is a system $\mathcal{T}$ whose term s are subcomplexes of the corresponding terms in $\mathcal{S}$ and whose maps are restrictions of the corresponding maps in $\mathcal{S}$. We also require that $\Gamma$ is the same for both systems and all the above group actions on the terms of $\mathcal{S}$ restrict to the actions by the same groups on the corresponding terms of $\mathcal{T}$. If $\mathcal{T}$ is a subsystem of $\mathcal{S}$, we will write $\mathcal{T} \subseteq \mathcal{S}$ and say that $(\mathcal{S}, \mathcal{T})$ is a pair of systems.

Since we assume that $\alpha$ and $\beta$ in a system $\mathcal{S}$ are immersions, we can always embed $\mathcal{S}$ as a subsystem of another system $\mathcal{S}^{\prime}$ as follows. Let $\alpha: Y^{\prime} \rightarrow X$ and $\beta: Z^{\prime} \rightarrow X$ be covers 
extending the above $\alpha$ and $\beta$. Let $\mathcal{S}^{\prime}$ be the system generated by $\alpha: Y^{\prime} \rightarrow X, \beta: Z^{\prime} \rightarrow X$, $p_{X}: \hat{X} \rightarrow X$, that is, $\mathcal{S}^{\prime}$ is the diagram

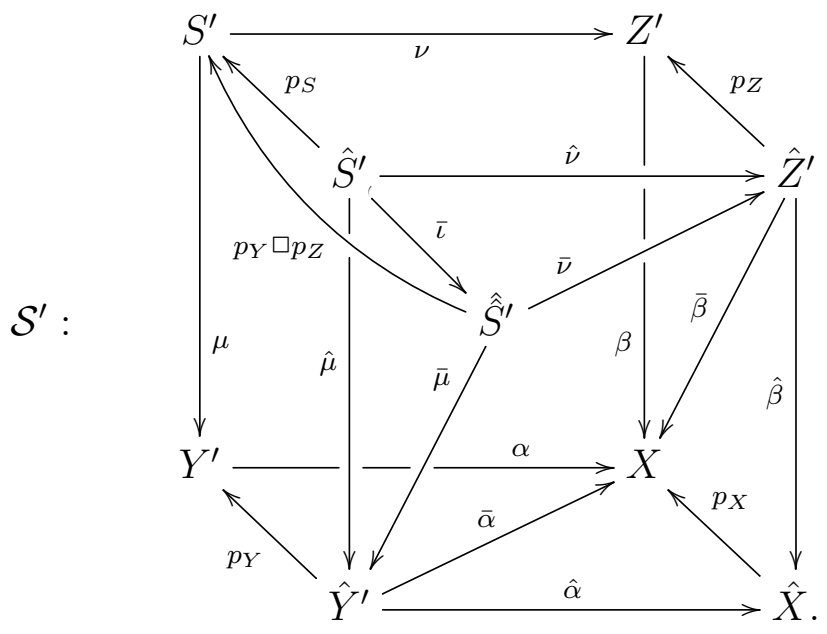

Then $\mathcal{S} \subseteq \mathcal{S}^{\prime}$. The following is an immediate corollary of Lemma 2 .

Lemma 6. For any pair of systems $\left(\mathcal{S}^{\prime}, \mathcal{S}\right)$ as above, the following hold.

- In the system $\mathcal{S}^{\prime}$, all the maps other than $\bar{\iota}$ are covers.

- In the system $\mathcal{S}$, all the maps are immersions. Furthermore, $p_{X}, p_{Y}, p_{Z}, p_{S}, p_{Y} \square p_{Z}$ are covers.

3.5. Properties of systems. We assume that $\hat{X}$ in a system is connected.

Theorem 7. Let $\mathcal{S}=\mathcal{S}\left(X, Y, Z, \hat{X}, \alpha, \beta, p_{X}\right)$ be a $\Gamma$-system of complexes as in (5).

(a) $\hat{X}$ is simply connected iff it is the universal cover of $X$.

Now additionally assume that $\hat{X}$ is simply connected.

(b) $\Gamma$ can be identified with $\pi_{1}(X)$.

(c) $\hat{\alpha}: \hat{Y} \rightarrow \hat{X}$ and $\hat{\beta}: \hat{Z} \rightarrow \hat{X}$ are leafages.

Now additionally assume that $Y$ and $Z$ are connected.

(d) If $\alpha: Y \rightarrow X$ is $\pi_{1}$-injective, or equivalently, the inclusion of $Y$ into the cover $Y^{\prime}$ is $\pi_{1}$-injective, then each component $K$ of $\hat{Y}$ is a copy of the universal cover of $Y$ and the restriction of $p_{Y}$ to $K, p_{Y}: K \rightarrow Y$, is the universal cover map. The same holds for $\left(Z, \hat{Z}, p_{Z}\right)$ in place of $\left(Y, \hat{Y}, p_{Y}\right)$.

(e) The induced $\Gamma$-actions on $\operatorname{Comp}(\hat{Y})$ and on $\operatorname{Comp}(\hat{Z})$ are transitive.

(f) For each $K \in \operatorname{Comp}(\hat{Y})$ its stabilizer $\operatorname{Stab}_{\Gamma}(K)$ is a conjugate of $\alpha_{*}\left(\pi_{1}(Y)\right)$ in $\Gamma$. The same holds for $(Z, \hat{Z}, \beta)$ in place of $(Y, \hat{Y}, \alpha)$.

(g) If $\alpha: Y \rightarrow X$ is a cover, then $\hat{\alpha}: \hat{Y} \rightarrow \hat{X}$ is a cover leafage. The same for $\hat{\beta}$.

Of course, all the above also applies to the system $\mathcal{S}^{\prime}$ as in (11).

Proof. Consider a system of complexes $\mathcal{S}$. We will view system $\mathcal{S}$ as embedded into a system $\mathcal{S}^{\prime}$ as in (11). 
(a) and (b) are standard facts from covering theory.

(c) Suppose $a$ and $b$ lie in the same component $K$ of $\hat{Y}$ and $\hat{\alpha}(a)=\hat{\alpha}(b)$, then there is a path $p$ in $K$ from $a$ to $b$ and $\hat{\alpha} \circ p$ is a loop in $\hat{X}$. Since $\hat{X}$ is simply connected, $\hat{\alpha} \circ p$ is nullhomotopic in $\hat{X}$. Since $\hat{\alpha}: \hat{Y}^{\prime} \rightarrow \hat{X}$ is a cover, this nullhomotopy lifts to a nullhomotopy of $p$ in $\hat{Y}^{\prime}$ (not necessarily in $\hat{Y}$ ). Then $p$ is a loop, so $a=b$.

(d) The equivalence of $\pi_{1}$-injectivity of $\alpha: Y \rightarrow X$ and of $Y \hookrightarrow Y^{\prime}$ follows from $\pi_{1}$-injectivity of $\alpha: Y^{\prime} \rightarrow X$.

By Lemma 6, $p_{Y}: \hat{Y} \rightarrow Y$ is a cover. Since cell complexes are locally path connected, the restricted map $p_{Y}: K \rightarrow Y$ is a cover. It remains to show that $K$ is simply connected. Take any loop $\hat{q}$ at any basepoint $\hat{y}_{0} \in K$. Since $\hat{X}$ is simply connected, the loop $\hat{p}:=\hat{\alpha} \circ \hat{q}$ is nullhomotopic in $\hat{X}$, hence the loop $p:=p_{X} \circ \hat{p}=p_{X} \circ \hat{\alpha} \circ \hat{q}$ is null-homotopic in $X$. Let $q:=p_{Y} \circ \hat{q}$, a loop in $Y$. Then $\alpha \circ q=p$. Since $\alpha: Y \rightarrow X$ is $\pi_{1}$-injective, there is a null-homotopy $h_{t}$ of $q$ in $Y, t \in[0,1]$. Then $f_{t}:=\alpha \circ h_{t}$ is a null-homotopy of $p$ in $X$, which lifts to a null-homotopy $\hat{f}_{t}$ of $\hat{p}$ in $\hat{X}$. The point-wise pull-back of $h$ and $\hat{f}$, denoted $h \square \hat{f}$, is a null-homotopy of $\hat{q}$ in $\hat{Y}$.
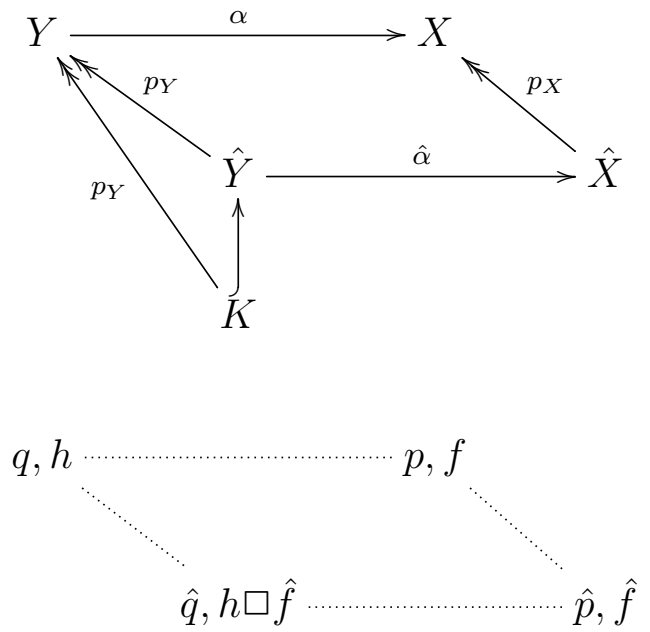

(e) Take any $K, L \in \operatorname{Comp}(\hat{Y})$ and any vertex $x$ in $K$. Since $Y$ is (path) connected, then the cover $p_{Y}: L \rightarrow Y$ is surjective. Then there exists a vertex $y \in L$ with $p_{Y}(y)=p_{Y}(x)$. By Lemma 5, $x$ and $y$ lie in the same $\Gamma$-orbit. Then some element of $\Gamma$ maps $x$ to $y$, and therefore $K$ onto $L$.

(f) The tacit assumption is that $X, \hat{X}, Y$ are given some basepoints $x_{0}, \hat{x}_{0}, y_{0}$ such that $\alpha\left(y_{0}\right)=x_{0}, p_{X}\left(\hat{x}_{0}\right)=x_{0}$. Let $\hat{y}_{0} \in \hat{Y}=Y \square \hat{X}$ be the pull-back of $y_{0}$ and $\hat{x}_{0}$, i.e. $\hat{y}_{0}:=\left(y_{0}, \hat{x}_{0}\right)$. Let $K$ be the component of $\hat{Y}$ containing $\hat{y}_{0}$. Let $K^{\prime}$ be the component of $\hat{Y}^{\prime}$ containing $K$.

For $g \in \pi_{1}(X)$, the point $g \hat{x}_{0} \in \hat{X}$ is defined by choosing any loop $p_{g}:[0,1] \rightarrow X$ representing $g$, lifting it to a path $\hat{p}$ starting at $\hat{x}_{0}$, and taking the other endpoint of $\hat{p}$, i.e. $g \hat{x}_{0}:=\hat{p}(1)$. This definition of course can be extended to define $g x$ for any $x \in \hat{X}$.

Take any $g \in \pi_{1}(X)$ and represent it by a loop $p_{g}$ at $x_{0}$ in $X$. Since $\alpha^{\prime}: Y^{\prime} \rightarrow X$ is a cover, $p_{g}$ lifts to a path $p^{\prime}$ in $Y^{\prime}$ starting at $y_{0} \in Y$. Let $\hat{p}$ be the lift of $p_{g}$ to $\hat{X}$. Let $\hat{p}^{\prime}:=p^{\prime} \square \hat{p}$, then 
$\hat{p}^{\prime}$ is a path in $\hat{Y}^{\prime}$ starting at $\hat{y}_{0} \in K$, hence $\hat{p}^{\prime}$ is a path in $K^{\prime}$. In particular, $\hat{p}^{\prime}(1) \in K^{\prime}$.
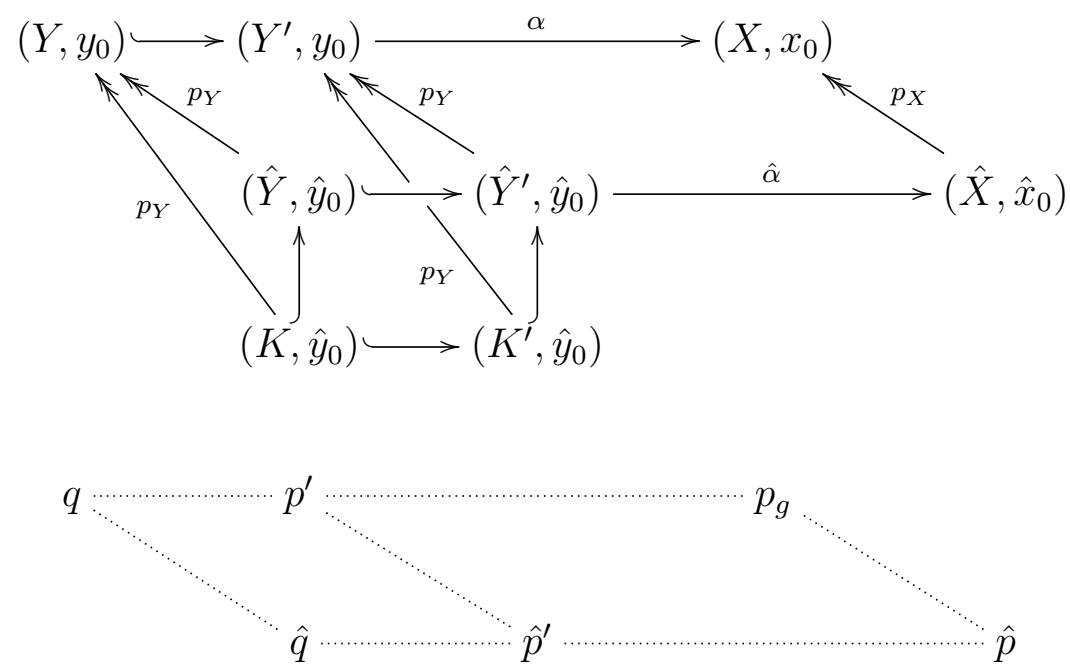

Since in the system $\mathcal{S}^{\prime}$

$$
g \in \operatorname{Stab}_{\Gamma}(K) \quad \Leftrightarrow \quad g \hat{y}_{0} \in K \Leftrightarrow \quad \hat{p}^{\prime}(1) \in K^{\prime} \text { and } g \hat{y}_{0} \in K
$$

$$
\hat{\alpha}\left(\hat{p}^{\prime}(1)\right)=\hat{p}(1)=g \hat{x}_{0}=g\left[\hat{\alpha}\left(\hat{y}_{0}\right)\right]=\hat{\alpha}\left(g \hat{y}_{0}\right),
$$

then (c) can be applied to the points $\hat{p}^{\prime}(1)$ and $g \hat{y}_{0}$ and component $K^{\prime}$ giving the equivalence

$$
\hat{p}^{\prime}(1) \in K^{\prime} \text { and } g \hat{y}_{0} \in K \quad \Leftrightarrow \quad \hat{p}^{\prime}(1)=g \hat{y}_{0} \in K
$$

By (d) applied to the system $\mathcal{S}^{\prime}, K^{\prime}$ is simply connected. Note also that

$$
p_{Y}\left(g \hat{y}_{0}\right)=y_{0}=p_{Y}\left(\hat{y}_{0}\right) \text {. }
$$

This implies the equivalences

$$
\begin{aligned}
& \hat{p}^{\prime}(1)=g \hat{y}_{0} \in K \\
& \Leftrightarrow \quad p^{\prime} \text { is a loop at } y_{0} \text { and } \\
& \quad \hat{p}^{\prime} \text { is homotopic in } \hat{Y}^{\prime} \text { to a path } \hat{q} \text { in } K \text { rel endpoints } \\
& \Leftrightarrow \quad p^{\prime} \text { is a loop at } y_{0} \text { and } \\
& \quad p^{\prime} \text { is homotopic in } Y^{\prime} \text { to a loop } q \text { in } Y \text { rel } y_{0} \\
& \Leftrightarrow \quad \text { there is a loop } q \text { in } Y \text { at } y_{0} \text { such that } \\
& \quad p_{g} \text { is homotopic to } \alpha \circ q \text { rel } x_{0} \\
& \Leftrightarrow \quad g \in \alpha_{*}\left(\pi_{1}(Y)\right) .
\end{aligned}
$$

Combining the above equivalences we obtain $\operatorname{Stab}_{\Gamma}(K)=\alpha_{*}\left(\pi_{1}(Y)\right)$. By (e), each component of $\hat{Y}$ is of the form $g K$ for some $g \in \Gamma$ and

$$
\operatorname{Stab}_{\Gamma}(g K)=g\left[\operatorname{Stab}_{\Gamma}(K)\right] g^{-1}=g\left[\alpha_{*}\left(\pi_{1}(Y)\right)\right] g^{-1} .
$$


(g) Suppose $K \in \operatorname{Comp}(\hat{Y})$. By (c), $\hat{\alpha}: K \rightarrow \hat{X}$ is injective. By (d), $p_{Y}: K \rightarrow Y$ is the universal cover, hence the map $p_{X} \circ \hat{\alpha}=\alpha \circ p_{Y}: K \rightarrow X$ is the universal cover. This implies that $\hat{\alpha}: K \rightarrow \hat{X}$ is an isomorphism. This finishes the proof of Theorem 7 .

Lemma 8. The induced actions by $\Gamma \times 1,1 \times \Gamma$, and $\Gamma \times \Gamma$ on $\operatorname{Comp}(\hat{X})$ are transitive.

Proof. The transitivity of the $\Gamma \times 1$-action follows by the argument of Theorem $7(\mathrm{e})$, replacing $Y$ with $\hat{X}$ and $\hat{Y}$ with $\hat{X}$. The transitivity of $1 \times \Gamma$-action is similar. This implies the transitivity of the $\Gamma \times \Gamma$-action.

3.6. Graph systems. A graph is a 1-dimensional cell complex. By a graph system we will mean a system $\mathcal{S}$ consisting of graphs. To avoid technicalities, in this paper we will always additionally assume that in a graph system

- $\Gamma$ is a free group of rank 2 ,

- $X$ is a finite graph with $\pi_{1}(X) \cong \Gamma$, for example $X$ can be the graph with one vertex and two edges,

- $Y$ and $Z$ are finite, and

- $p_{X}: \hat{X} \rightarrow X$ is the universal cover map.

3.7. Connected graph systems. Recall that a system is called connected if $X, Y$ and $Z$ are connected. We show how the assumptions of SHNC give rise to a connected graph system, and vice versa.

Let $\Gamma$ be the free group of rank 2. Pick any finitely generated subgroups $A$ and $B$ of $\Gamma$, let $\alpha^{\prime}: Y^{\prime} \rightarrow X$ and $\beta^{\prime}: Z^{\prime} \rightarrow X$ be covers with $\pi_{1}\left(Y^{\prime}\right)=A$ and $\pi_{1}\left(Z^{\prime}\right)=B$. Some basepoints in $X^{\prime}, Y^{\prime}, Z^{\prime}$ are assumed here. Note that this means the graphs $Y^{\prime}$ and $Z^{\prime}$ are connected. Since $A$ and $B$ are finitely generated, there are (connected) finite subgraphs $Y \subseteq Y^{\prime}$, $Z \subseteq Z^{\prime}$ that are deformation retracts of $Y^{\prime}$ and $Z^{\prime}$, respectively, and contain the basepoints. Let $\alpha: Y \rightarrow X$ and $\beta: Z \rightarrow X$ be restrictions of $\alpha^{\prime}$ and $\beta^{\prime}$. Then $\pi_{1}(Y)=A, \pi_{1}(Z)=B$, and the induced homomorphisms $\pi_{1}(\alpha): \pi_{1}(Y) \hookrightarrow \pi_{1}(X)$ and $\pi_{1}(\beta): \pi_{1}(Z) \hookrightarrow \pi_{1}(X)$ are the subgroup inclusions $A \subseteq \Gamma$ and $B \subseteq \Gamma$. This construction is described by Stallings [24] in the category of Serre graphs; it can be easily adjusted to our topological setting of 1-dimensional cell complexes.

Consider the connected graph system $\mathcal{S}$ generated by the above $\alpha, \beta$ and $p_{X}$ as in 3.1. By Theorem $7(\mathrm{f})$, the stabilizers of the components of $\hat{Y}$ are the conjugates of $A$ in $\Gamma$, and the stabilizers of the components of $\hat{Z}$ are the conjugates of $B$ in $\Gamma$.

It is possible to extend the above $\alpha$ and $\beta$ to connected finite covers $\alpha: Y^{\circ} \rightarrow X$ and $\beta: Z^{\circ} \rightarrow X$. This is shown by adjusting the proof of [24, Theorem 6.1] to our topological setting. These maps generate the following system $\mathcal{S}^{\circ}$ containing $\mathcal{S}$ as a subsystem. 


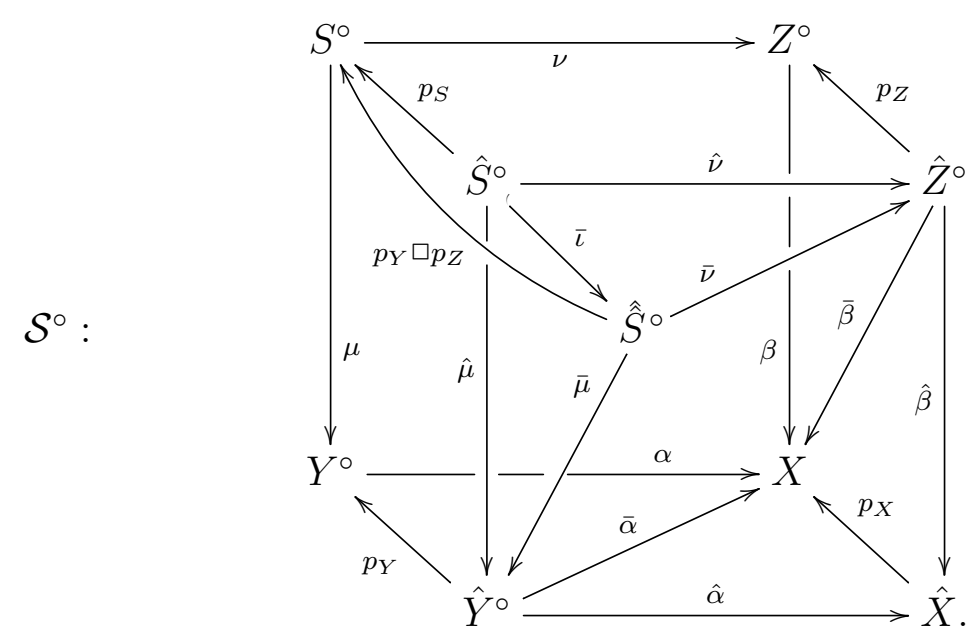

Set $A^{\circ}:=\pi_{1}\left(Y^{\circ}\right)$ and $B^{\circ}:=\pi_{1}\left(Z^{\circ}\right)$. We will identify $A^{\circ}$ and $B^{\circ}$ with subgroups of $\Gamma$. They are of finite index since $Y^{\circ}$ and $Z^{\circ}$ are finite covers of $X$. By Theorem $7(\mathrm{f})$, the stabilizers of the components of $\hat{Y}^{\circ}$ are the conjugates of $A^{\circ}$ in $\Gamma$, and the stabilizers of the components of $\hat{Z}^{\circ}$ are the conjugates of $B^{\circ}$ in $\Gamma$.

\section{Groups And Hilbert modules.}

4.1. Hilbert $\Gamma$-modules. We collect standard facts about Hilbert modules; see [15] for a detailed exposition.

All Hilbert spaces will be over $\mathbb{C}$. A Hilbert $\Gamma$-module is a Hilbert space $V$ isometrically isomorphic to a $\Gamma$-invariant Hilbert subspace of $\ell^{2}(\Gamma) \otimes U$, where $U$ is some Hilbert space given the trivial $\Gamma$-action. This gives a $\Gamma$-action on $V$ which it turn gives an $\mathcal{N}(\Gamma)$-module structure on $V$, where $\mathcal{N}(\Gamma)$ is the von Neumann algebra of $\Gamma$. A morphism of Hilbert $\Gamma$-modules is a bounded map that commutes with the left $\Gamma$-action. A morphism of Hilbert $\Gamma$-modules is a weak isomorphism if it is injective and has dense image.

For a set $S, \ell^{2}(S)$ is the Hilbert space with orthonormal basis $S$. If $S$ is a free $\Gamma$-set, the (left) $\Gamma$-action on $\ell^{2}(S)$ is defined by

$$
(g f)(s):=f\left(g^{-1} s\right) \quad \text { for } g \in \Gamma, f \in \ell^{2}(S), s \in S,
$$

giving $\ell^{2}(S)$ the structure of a Hilbert $\Gamma$-module.

Any Hilbert $\Gamma$-module $V$ is assigned the Murray-von Neumann dimension by the rule

$$
\operatorname{dim}_{\Gamma}\left(V^{\prime}\right):=\sum_{i=1}^{n}\left\langle p r_{V^{\prime}}\left(b_{i} \otimes e\right), b_{i} \otimes e\right\rangle,
$$

where $V^{\prime} \subseteq \ell^{2}(\Gamma) \otimes U$ is any isometric copy of $V, U$ is a Hibert space, $e \in \Gamma \subseteq \ell^{2}(\Gamma)$ is the identity element, $B \subseteq V$ is an orthonormal basis of the Hilbert space $V$, and $p r_{V^{\prime}}$ is the orthogonal projection of $\ell^{2}(\Gamma) \otimes U$ onto $V^{\prime}$. 
The dimension $\operatorname{dim}_{\Gamma}$ is additive in the following sense: if

$$
0 \rightarrow U \rightarrow V \rightarrow W \rightarrow 0
$$

is a weakly exact sequence of Hilbert $\Gamma$-modules, then $\operatorname{dim}_{\Gamma} V=\operatorname{dim}_{\Gamma} U+\operatorname{dim}_{\Gamma} W[15$, Theorem $1.12(2)]$.

Lück defined a notion of dimension for arbitrary (purely algebraic) modules over $\mathcal{N}(\Gamma)$ that extends the Murray-von Neumann dimension [15, Definition 6.20]. If $f: U \subseteq V$ is an $\mathcal{N}(\Gamma)$ submodule of a Hilbert $\Gamma$-module $V$, then one can define $\operatorname{dim}_{\Gamma} U$ by the Lück's extended definition. This is equivalent to setting $\operatorname{dim}_{\Gamma} U:=\operatorname{dim}_{\Gamma} \bar{U}$.

4.2. $\ell^{2}$-homology and $\ell^{2}$-Betti numbers. Given a free $\Gamma$-complex $\hat{X}$, let $\Sigma_{i}(\hat{X})$ be the set of $i$-cells in $\hat{X}$ and $C_{i}^{(2)}(\hat{X}):=\ell^{2}\left(\Sigma_{i}(\hat{Z})\right)$.

The $\ell^{2}$-homology of $\hat{X}$ is the reduced homology of the chain complex

$$
\cdots \stackrel{\partial_{i+2}}{\longrightarrow} C_{i+1}^{(2)}(\hat{X}) \stackrel{\partial_{i+1}}{\longrightarrow} C_{i}^{(2)}(\hat{X}) \stackrel{\partial_{i}}{\longrightarrow} C_{i-1}^{(2)}(\hat{X}) \stackrel{\partial_{i-1}}{\longrightarrow} \cdots,
$$

that is $H_{i}^{(2)}(\hat{X}):=\operatorname{Ker} \partial_{i} / \overline{\operatorname{Im} \partial_{i+1}}$. Here $\partial_{i}$ is the completion of the usual boundary map $C_{i}(\hat{X}) \rightarrow C_{i-1}(\hat{X})$. Note that $\hat{X}$ is not assumed to be connected.

The $i$ th $\ell^{2}$-Betti number of $(\hat{X}, \Gamma)$ is

$$
b_{i}^{(2)}(\hat{X} ; \Gamma):=\operatorname{dim}_{\Gamma} H_{i}^{(2)}(\hat{X}) .
$$

Notation. We will write $\mathrm{H}(\hat{X})$ instead of $H_{1}^{(2)}(\hat{X})$.

When $\hat{X}$ is a graph, $\mathrm{H}(\hat{X})$ is the same as the set of 1 -dimensional $\ell^{2}$-cycles, and we will always view it as a subspace of $C_{1}^{(2)}(\hat{X})$. Functor $\mathrm{H}(\cdot)$ preserves injectivity: if $\hat{X} \subseteq \hat{X}^{\circ}$ are graphs then

$$
\begin{aligned}
& \mathrm{H}(\hat{X})=\operatorname{Ker}\left[\partial: C_{1}^{(2)}(\hat{X}) \rightarrow C_{0}^{(2)}(\hat{X})\right]=\operatorname{Ker}\left[\partial: C_{1}^{(2)}(\hat{X}) \rightarrow C_{0}^{(2)}\left(\hat{X}^{\circ}\right)\right] \\
& =\operatorname{Ker}\left[\partial: C_{1}^{(2)}\left(\hat{X}^{\circ}\right) \rightarrow C_{0}^{(2)}\left(\hat{X}^{\circ}\right)\right] \cap C_{1}^{(2)}(\hat{X}) \subseteq \mathrm{H}\left(\hat{X}^{\circ}\right) \subseteq C_{1}^{(2)}\left(\hat{X}^{\circ}\right) .
\end{aligned}
$$

4.3. Induction. If $G$ is a subgroup of a group $\Gamma$ and $X$ is a complex with a free $G$-action, denote

$$
\operatorname{Ind}_{G}^{\Gamma} X:=\bigsqcup_{a \in \Gamma / G} a X \cong(\Gamma / G) \times X
$$

where aX is a copy of $X$. Then $\operatorname{Ind}_{G}^{\Gamma}(X)$ can be given a $\Gamma$-set structure. If $V$ is a Hilbert $G$-module, denote $\operatorname{Ind}_{G}^{\Gamma} V$ the completed orthogonal sum $\hat{\oplus}_{a \in \Gamma / G} a V$, where $a V$ is a copy of $V$. Then $\operatorname{Ind}_{G}^{\Gamma}(V)$ can be given a Hilbert $\Gamma$-module structure and it follows that

$$
\operatorname{dim}_{\Gamma} \operatorname{Ind}_{G}^{\Gamma} V=\operatorname{dim}_{G} V
$$

(see [15, Lemma 1.24]).

Lemma 9. For any system $\mathcal{S}$ there are $\Gamma \times \Gamma$-isomorphisms

(a) $\hat{X} \cong \operatorname{Ind}_{\Delta}^{\Gamma \times \Gamma} \hat{X}_{\Delta}$ and

(b) $\hat{S} \cong \operatorname{Ind}_{\Delta}^{\Gamma \times \Gamma} \hat{S}$. 
Proof. This follows from lemmas 4 and 8.

4.4. Bases. Let $\mathcal{B}$ be a set. The action of $\Gamma$ on itself induces a $\Gamma$-action on $\Gamma \mathcal{B}$ which extends to a Hilbert $\Gamma$-module structure on $\ell^{2}(\Gamma \times \mathcal{B})$ and also to an $\mathcal{N}(\Gamma)$-module structure on $\ell^{2}(\Gamma \times \mathcal{B})$. The standard orthonormal $\Gamma$-invariant basis of $\ell^{2}(\Gamma \times \mathcal{B})$ is the set $\Gamma \times \mathcal{B}$. This is not to be confused with the standard orthonormal $\Gamma$-basis which is the set $\mathcal{B}=1 \times \mathcal{B}$.

If $V$ is a Hilbert $\Gamma$-module, an orthonormal $\Gamma$-invariant basis of $V$ is the image of $\Gamma \times \mathcal{B}$ under a Hilbert $\Gamma$-module map $\ell^{2}(\Gamma \times \mathcal{B}) \rightarrow V$ that is an isometric isomorphism, for some $\mathcal{B}$. Similarly, an orthonormal $\Gamma$-basis of $V$ is the image of $\mathcal{B}=1 \times \mathcal{B}$ under such an isometric isomorphism.

A $\Gamma$-invariant basis of $V$ is the image of $\Gamma \times \mathcal{B}$ under a Hilbert $\Gamma$-module map $\ell^{2}(\Gamma \times \mathcal{B}) \rightarrow V$ that is a weak isomorphism, for some $\mathcal{B}$. Similarly, a $\Gamma$-basis of $V$ is the image of $\mathcal{B}=1 \times \mathcal{B}$ under such a weak isomorphism.

A $\Gamma$-subbasis of a Hilbert $\Gamma$-module $V$ is a subset of some $\Gamma$-basis. An orthonormal $\Gamma$-subbasis of $V$ is a subset of some orthonormal $\Gamma$-basis.

4.5. Change of Hilbert structure. Suppose $f: V \rightarrow V^{\prime}$ is a Hilbert $\Gamma$-module map that is a weak isomorphism. By the change of Hilbert structure on $V$ by $f$ we mean replacing $V$ with $V^{\prime}$ and $f$ with the identity map $V^{\prime} \rightarrow V^{\prime}$. Equivalently, this means that for $v, w \in V$, the inner product $\langle v, w\rangle$ is redefined to be $\langle f(v), f(w)\rangle$ and then $V$ is completed with respect to this new inner product. Similarly, by the change of Hilbert structure on $V^{\prime}$ by $f$ we mean replacing $V^{\prime}$ with $V$ and $f$ with the identity map $V \rightarrow V$. Equivalently, $V^{\prime}$ is replaced with $f(V)$ and is given the Hilbert structure induced by $f$ from $V$. A change of Hilbert structure on a Hilbert $\Gamma$-module is the result of applying a finite sequence of changes as above. This change preserves dimension.

4.6. The Atiyah conjecture. For the moment, let $\Gamma$ be any torsion-free group and $M$ be an $m \times n$-matrix with entries in $\mathbb{C} \Gamma$. The right multiplication by $A$ gives a $\Gamma$-equivariant bounded $\operatorname{map} \tau_{M}: \ell^{2}(\Gamma)^{m} \rightarrow \ell^{2}(\Gamma)^{n}$. The (integral version of) Atiyah conjecture states that $\operatorname{dim}_{\Gamma} \operatorname{Ker} \tau_{M}$ is an integer. It came from a question by Atiyah in [1].

Linnell proved in [14] that the Atiyah conjecture holds for free groups and, more generally, for extensions $0 \rightarrow H \rightarrow \Gamma \rightarrow \Gamma / H \rightarrow 0$ where $H$ is free and $\Gamma / H$ is elementary amenable. This in particular includes the infinite surface groups since $H$ can be taken to be the commutator of $\Gamma$.

4.7. Lifts. A family $\mathcal{F}$ of functions on a cell complex $\hat{Y}$ will be said to be inscribed in $\operatorname{Comp}(\hat{Y})$ if for each $f \in \mathcal{F}$ there exists $K \in \operatorname{Comp}(\hat{X})$ such that $\operatorname{supp}(f) \subseteq K$.

Suppose $p: \hat{Y} \rightarrow \hat{X}$ is a leafage as in 2.4, a family $\mathcal{F} \subseteq C_{i}^{(2)}(\hat{X})$ is inscribed in $\operatorname{Comp}(\hat{X})$, and $\mathcal{F}$ does not contain the zero function. For $f \in \mathcal{F}$, let $K(f)$ denote the component of $\hat{X}$ containing $\operatorname{supp}(f)$. Define the lift of $\mathcal{F}$ to $\hat{Y}$ via $p$ as

$$
p^{\uparrow}(\mathcal{F}):=\left\{f_{L} \mid f \in \mathcal{F}, L \in \operatorname{Comp}(\hat{Y}), p(L)=K(f)\right\} \subseteq C_{i}^{(2)}(\hat{Y}),
$$

where $f_{L}: \hat{Y} \rightarrow \mathbb{C}$ is defined on $L$ to be the restriction of $f \circ p$ to $L$ and zero on any other component of $\hat{Y}$. Then $p^{\uparrow}(\mathcal{F})$ is inscribed into $\operatorname{Comp}(\hat{Y})$. 
4.8. Matrix stacking. Suppose $U, V$, and $W$ are finite orthogonal sums of copies of $\ell^{2}(\Gamma), M$ and $N$ are finite matrices with entries in $\mathbb{C} \Gamma$, and $\tau_{M}: U \rightarrow V$ and $\tau_{N}: U \rightarrow V^{\prime}$ are the maps given by the right multiplication with $M$ and $N$, respectively. Let $[M \mid N]$ be the matrix obtained by placing $M$ and $N$ horizontally next to each other. The right multiplication by $[M \mid N]$ gives a $\operatorname{map} \tau_{[M \mid N]}: U \rightarrow V \oplus V^{\prime}$ which we also denote $\left[\tau_{M} \mid \tau_{N}\right]$. Clearly, $\operatorname{Ker}\left[\tau_{M} \mid \tau_{N}\right]=\operatorname{Ker} \tau_{M} \cap \operatorname{Ker} \tau_{N}$.

Similarly, if $\tau_{M}: U \rightarrow V$ and $\tau_{N}: U^{\prime} \rightarrow V$ are given by matrices $M$ and $N$, the map $\left[\frac{\tau_{M}}{\tau_{N}}\right]: U \oplus U^{\prime} \rightarrow V$ is given by stacking the matrices $M$ and $N$ vertically. Then $\operatorname{Im}\left[\frac{\tau_{M}}{\tau_{N}}\right]=$ $\operatorname{Im} \tau_{M}+\operatorname{Im} \tau_{N}$.

An obvious but important observation is that stacking two matrices with entries in $\mathbb{C} \Gamma$ produces a martix with entries again in $\mathbb{C} \Gamma$.

Theorem 10. Let $\Gamma$ be a group satisfying the Atiyah conjecture, $M, P, Q$ be finite orthogonal sums of copies of $\ell^{2}(\Gamma)$, and $f: M \rightarrow P$ and $g: M \rightarrow Q$ be Hilbert $\Gamma$-module maps such that for each element $e$ of the standard $\Gamma$-basis in $M, f(e)$ and $g(e)$ are finite linear combinations of elements of the standard $\Gamma$-invariant bases of $P$ and $Q$, respectively. Then

(1) $\operatorname{dim}_{\Gamma}[\operatorname{Ker} f \cap \operatorname{Ker} g] \in \mathbb{Z}$ and

(2) $\operatorname{dim}_{\Gamma}\left[(\operatorname{Ker} f)^{\perp} \cap \operatorname{Ker} g\right] \in \mathbb{Z}$.

Proof. The assumptions imply that the maps $f: M \rightarrow P$ and $g: M \rightarrow Q$ are given by the right multiplication with finite matrices whose entries are in $\mathbb{C} \Gamma$.

(1) follows immediately from horizontal matrix stacking and the Atiyah conjecture.

(2) Denote $h:=f^{*} \circ f: M \rightarrow M$. Since $h$ is selfadjoint, we have

$$
\overline{\operatorname{Im} h}=(\operatorname{Ker} h)^{\perp}=(\operatorname{Ker} f)^{\perp},
$$

where the orthogonal complements and closures are taken in $M$. Then

$$
\begin{aligned}
& (\operatorname{Ker} f)^{\perp} \cap \operatorname{Ker} g=\overline{\operatorname{Im} h} \cap \operatorname{Ker} g, \\
& h^{-1}(\overline{\operatorname{Im} h} \cap \operatorname{Ker} g)=h^{-1}(\operatorname{Im} h \cap \operatorname{Ker} g)=\operatorname{Ker}(g \circ h) .
\end{aligned}
$$

Since $\Gamma$ satisfies the Atiyah conjecture,

$$
\begin{aligned}
& \operatorname{dim}_{\Gamma} \operatorname{Ker} h \in \mathbb{Z}, \\
& \operatorname{dim}_{\Gamma} h^{-1}(\operatorname{Im} h \cap \operatorname{Ker} g)=\operatorname{dim}_{\Gamma} \operatorname{Ker}(g \circ h) \in \mathbb{Z} .
\end{aligned}
$$

Since $\operatorname{Im} h \cap \operatorname{Ker} g \subseteq \operatorname{Im} h$, the sequence of $\mathcal{N}(\Gamma)$-modules

$$
0 \rightarrow \operatorname{Ker} h \hookrightarrow h^{-1}(\operatorname{Im} h \cap \operatorname{Ker} g) \stackrel{h}{\rightarrow} \operatorname{Im} h \cap \operatorname{Ker} g \rightarrow 0
$$

is exact, then

$$
\operatorname{dim}_{\Gamma}[\operatorname{Im} h \cap \operatorname{Ker} g]=\operatorname{dim}_{\Gamma}\left[h^{-1}(\operatorname{Im} h \cap \operatorname{Ker} g)\right]-\operatorname{dim}_{\Gamma} \operatorname{Ker} h \in \mathbb{Z} .
$$

In the diagram

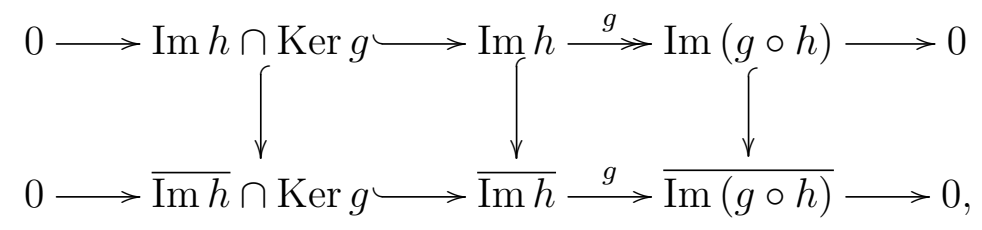


the bottom row consists of Hilbert $\Gamma$-modules and is weakly exact, and the top row consists of $\mathcal{N}(\Gamma)$-modules and is exact. The last two vertical arrows preserve dimension, hence by additivity the first vertical arrow does as well, hence

$$
\operatorname{dim}_{\Gamma}\left[(\operatorname{Ker} f)^{\perp} \cap \operatorname{Ker} g\right]=\operatorname{dim}_{\Gamma}[\overline{\operatorname{Im} h} \cap \operatorname{Ker} g]=\operatorname{dim}_{\Gamma}[\operatorname{Im} h \cap \operatorname{Ker} g] \in \mathbb{Z} .
$$

The first homology of a graph is the kernel of the boundary map in dimension 1 . Theorem 10 is a statement about kernels, and it will be used for graphs (theorems 16 and 29 below). We will also want to consider surfaces and complexes. For them, the following more general statement will be needed (in Theorem 26).

Theorem 11. Suppose the diagram

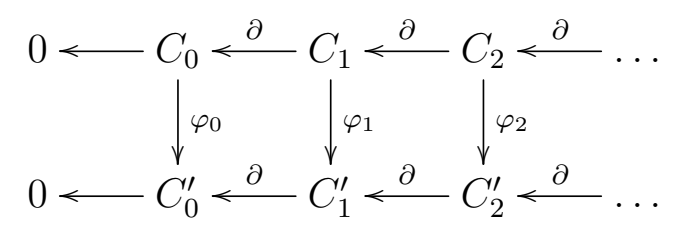

is commutative, its rows are chain complexes of Hilbert $\Gamma$-modules, each term is a finite sum of several copies of $\ell^{2}(\Gamma)$, and each arrow is given by the right multiplication by a finite matrix with entries in $\mathbb{C} \Gamma$. If $\Gamma$ satisfies the Atiyah conjecture and $\psi_{n}: \bar{H}_{n}\left(C_{*}\right) \rightarrow \bar{H}_{n}\left(C_{*}^{\prime}\right)$ is the map induced by $\varphi_{*}$ on the $n$th $\ell^{2}$-homology, then $\operatorname{dim}_{\Gamma} \operatorname{Ker} \psi_{n} \in \mathbb{Z}$.

Proof. The best proof is by a spectral sequence. Replace $\varphi_{n}$ with $\pm \varphi_{n}$ appropriately so that the diagram becomes anticommutative, and extend $\partial$ and $\varphi$ by zeros so that it can be viewed as a first-quadrant double complex. The total complex is the chain $\operatorname{complex}$ Tot $_{*}$ with Tot $_{n}=$ $C_{n}^{\prime} \oplus C_{n-1}$ and the total differential $D:=\partial+\varphi$. The fact that the spectral sequence for this double complex converges to the $\ell^{2}$-homology of Tot ${ }_{*}$ holds by applying the usual proof to the category of Hilbert modules and taking closures of images rather than images in appropriate places. Alternatively, one can work in the category of $\mathcal{N}(\Gamma)$-modules, take homology in the usual algebraic way, and disregard modules of dimension 0.

The zeroth differential in the spectral sequence is the horizontal map $\partial$, so the first page is

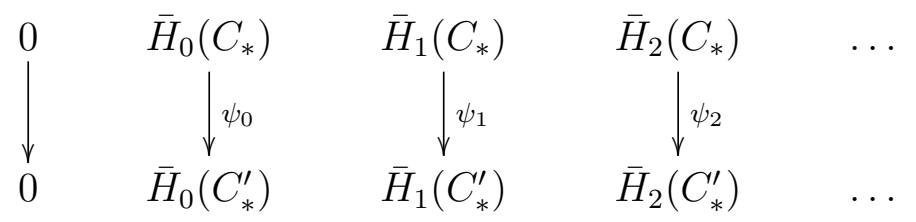

on which the first differential is the vertical map $\psi$. Taking the $\ell^{2}$-homology with respect to $\psi$ gives the second page

$\begin{array}{ccccc}0 & \operatorname{Ker} \psi_{0} & \operatorname{Ker} \psi_{1} & \operatorname{Ker} \psi_{2} & \ldots \\ 0 & \overline{\operatorname{Coker}} \psi_{0} & \overline{\operatorname{Coker}} \psi_{1} & \overline{\operatorname{Coker}} \psi_{2} & \ldots\end{array}$


on which the spectral sequence collapses. Here $\overline{\operatorname{Coker}} \psi_{n}:=\bar{H}_{n}\left(C_{*}^{\prime}\right) / \overline{\operatorname{Im} \psi_{n}}$. Then

$$
\bar{H}_{n}\left(\operatorname{Tot}_{*}\right) \cong \overline{\operatorname{Coker}} \psi_{n} \oplus \operatorname{Ker} \psi_{n-1} .
$$

The total differential $D$ is given by the right multiplication with a finite matrix with entries in $\mathbb{C} \Gamma$. (This can be viewed as "multiple matrix stacking".) Since $\Gamma$ satisfies the Atiyah conjecture, this implies that $\operatorname{dim}_{\Gamma} \operatorname{Ker} D_{n} \in \mathbb{Z}$ and $\operatorname{dim}_{\Gamma} \operatorname{Im} D_{n} \in \mathbb{Z}$ for each $n$. Thus

$$
\operatorname{dim}_{\Gamma} \overline{\operatorname{Coker}} \psi_{n} \oplus \operatorname{Ker} \psi_{n-1}=\operatorname{dim}_{\Gamma} \bar{H}_{n}\left(\operatorname{Tot}_{*}\right) \in \mathbb{Z} .
$$

For $n=0$ this means that $\operatorname{dim}_{\Gamma} \overline{\text { Coker }} \psi_{0} \in \mathbb{Z}$. This together with

$$
\operatorname{dim}_{\Gamma} \overline{\operatorname{Coker}} \psi_{1} \oplus \operatorname{Ker} \psi_{0}=\operatorname{dim}_{\Gamma} \bar{H}_{1}\left(\operatorname{Tot}_{*}\right) \in \mathbb{Z}
$$

implies that $\operatorname{dim}_{\Gamma} \operatorname{Ker} \psi_{0} \in \mathbb{Z}$. Continue inductively using (14) to deduce that $\operatorname{dim}_{\Gamma} \operatorname{Ker} \psi_{n} \in \mathbb{Z}$ for all $n$.

\section{Graphs.}

In this section we discuss relations among graphs, reduced rank, and $\ell^{2}$-Betti numbers.

5.1. Flowers and gardens. Recall that by a graph we mean a 1-complex. A tree is a graph homotopy equivalent to a point. A forest is a graph whose connected components are trees.

A flower will be a graph homotopy equivalent to a circle. A garden will be a graph such that each of its connected components is a tree or a flower. A subgarden of a graph $Z$ is a subgraph that is a garden. A maximal subgarden of a graph $Z$ is a subgarden maximal with respect to inclusions.

5.2. The reduced rank of graphs. For a finite graph $Y$, define its reduced rank by

$$
\bar{r}(Y):=\sum_{K \in \operatorname{Comp}(Y)} \max \{0,-\chi(K)\}
$$

in analogy with (1). This includes the empty case $\bar{r}(\emptyset)=0$. If $Y$ is non-empty, one checks that

$$
\bar{r}(Y)=\sum_{K \in \operatorname{Comp}(Y)} \max \left\{0, \operatorname{rk}\left(\pi_{1}(K)\right)-1\right\}=\sum_{K \in \operatorname{Comp}(Y)} \bar{r}\left(\pi_{1}(K)\right) .
$$

5.3. Essential sets. Let $Z$ be any finite graph. An edge $\sigma \in \Sigma_{1}(Z)$ will be called essential in $Z$ if $\bar{r}(Z \backslash\{\sigma\})=\bar{r}(Z)-1$. More generally, a set of edges $E \subseteq \Sigma_{1}(Z)$ is essential in $Z$ if $\bar{r}(Z \backslash E)=\bar{r}(Z)-\# E$. A maximal essential set is an essential set that is maximal with respect to inclusions. For an edge $\sigma$, denote $\sigma^{-}$and $\sigma^{+}$the initial and the terminal vertices of $\sigma$, respectively. The following lemma is an easy exercise and will not be used in this paper, so we skip the proof.

Lemma 12. Suppose $Z$ is a finite graph and $E \subseteq \Sigma_{1}(Z)$. The following statements are equivalent.

(a) $E$ is an essential set.

(b) For each $e \in E$, the component of $Z \backslash E$ containing $\sigma^{-}$is not a tree and the component of $Z \backslash E$ contaning $\sigma^{+}$is not a tree. 
And the following statements are equivalent.

$\left(a^{\prime}\right) E$ is a maximal essential set.

$\left(b^{\prime}\right) \bar{r}(Z \backslash E)=0$ and for each $e \in E$, the component of $Z \backslash E$ containing $\sigma^{-}$is not a tree and the component of $Z \backslash E$ contaning $\sigma^{+}$is not a tree.

$\left(c^{\prime}\right) Z \backslash E$ is a maximal subgarden of $Z$.

$\left(d^{\prime}\right) \bar{r}(Z \backslash E)=0=\bar{r}(Z)-\# E$.

The equivalence of $\left(c^{\prime}\right)$ and $\left(d^{\prime}\right)$ shows that maximal subgardens play the same role for the first $\ell^{2}$-Betti numbers as maximal subtrees play for the usual first Betty numbers.

5.4. $\ell^{2}$-Betti numbers for graphs. The integrality of $\ell^{2}$-Betti numbers for free groups can be deduced from the Atiyah conjecture for free groups by Linnell's result [14]. We present an elementary proof of integrality and an explicit description of the numbers.

Lemma 13. Suppose $\Gamma$ is a group.

(a) If $\Gamma$ acts freely and cocompactly on a graph $\hat{Y}$ whose components are infinite, then

$$
b_{0}^{(2)}(\hat{Y} ; \Gamma)=0 \quad \text { and } \quad b_{1}^{(2)}(\hat{Y} ; \Gamma)=-\chi(\Gamma \backslash \hat{Y}) .
$$

(b) If $\Gamma$ acts freely and cocompactly on a graph $\hat{Y}$ whose components are finite and the stabilizer of each component of $\hat{Y}$ is trivial, then

$$
b_{i}^{(2)}(\hat{Y} ; \Gamma)=b_{i}(\Gamma \backslash \hat{Y})
$$

for all $i$, where $b_{i}(\Gamma \backslash \hat{Y})$ are the usual Betti numbers of $\Gamma \backslash \hat{Y}$.

(c) If $\Gamma$ is torsion-free and acts freely and cocompactly on a graph $\hat{Y}$ then all the $\ell^{2}$-Betti numbers $b_{i}^{(2)}(\hat{Y} ; \Gamma)$ are integers.

Proof. (a) Let $Y:=\Gamma \backslash \hat{Y}, q: \hat{Y} \rightarrow Y$ be the quotient map, then

$$
Y=\bigsqcup_{K \in \operatorname{Comp}(Y)} K, \quad \hat{Y}=\bigsqcup_{K \in \operatorname{Comp}(Y)} q^{-1}(K), \quad \text { and } \quad q^{-1}(K) / \Gamma=K .
$$

Each term in (15) is additive under disjoint unions, so it suffices to prove the statement assuming that $Y$ is connected. In this case the induced $\Gamma$-action on $\operatorname{Comp}(\hat{Y})$ is transitive. Pick any $L \in \operatorname{Comp}(\hat{Y})$ and denote $\Gamma_{L}:=\operatorname{Stab}_{\Gamma}(L)$, then $Y=\Gamma \backslash \hat{Y}=\Gamma_{L} \backslash L$, so $q: L \rightarrow Y$ is a connected cover. There is an isomorphism of $\Gamma$-sets $\operatorname{Comp}(\hat{Y}) \cong \Gamma / \Gamma_{L}$ which induces an isomorphism of $\Gamma$-graphs

$$
\hat{Y} \cong \operatorname{Comp}(\hat{Y}) \times L \cong\left(\Gamma / \Gamma_{L}\right) \times L \cong \operatorname{Ind}_{\Gamma_{L}}^{\Gamma} L
$$

and consequently an isomorphism of Hilbert $\Gamma$-modules

$$
C_{*}^{(2)}(\hat{Y}) \cong \operatorname{Ind}_{\Gamma_{L}}^{\Gamma} C_{*}^{(2)}(L) .
$$

Since induction commutes with homology,

$$
H_{*}^{(2)}(\hat{Y}) \cong \operatorname{Ind}_{\Gamma_{L}}^{\Gamma} H_{*}^{(2)}(L) .
$$


Since induction preserves dimension [15, Lemma 1.24],

$$
\operatorname{dim}_{\Gamma_{L}} \mathrm{H}(L)=\operatorname{dim}_{\Gamma} \mathrm{H}(\hat{Y}) .
$$

The usual Euler characteristic $\chi(Y)$ can be computed as the alternating sum of $\ell^{2}$-Betti numbers for the pair $\left(L, \Gamma_{L}\right)[15$, Theorem $1.35(2)]$, so

$$
\chi(X)=b_{0}^{(2)}\left(L ; \Gamma_{L}\right)-b_{1}^{(2)}\left(L ; \Gamma_{L}\right) .
$$

Since $L$ is an infinite connected graph, it is an easy exercise to see that every $\ell^{2}$-summable 0-chain in $L$ can be approximated by the boundaries of finitely supported 1-chains in $L$. (Or see [15, Theorem 1.35(8)].) Therefore, $b_{0}^{(2)}\left(L ; \Gamma_{L}\right)=0$, which implies (15).

(b) The argument as in (1) shows that $Y:=\Gamma \backslash \hat{Y}$ is connected, $Y=\Gamma_{L} \backslash L$, where $L \in \operatorname{Comp}(\hat{Y})$ and $\Gamma_{L}:=\operatorname{Stab}_{L}(\Gamma)$. Since $\Gamma_{L}$ is trivial then $Y=L$ and

$$
b_{i}^{(2)}(\hat{Y} ; \Gamma)=b_{i}^{(2)}\left(L ; \Gamma_{L}\right)=\operatorname{dim}_{\Gamma_{L}} H_{i}^{(2)}(L)=\operatorname{dim} H_{i}(L ; \mathbb{C})=b_{i}(Y) .
$$

(c) The stabilizers of the finite components of $\hat{Y}$ are finite, hence trivial. Now the statement follows from (a) and (b) by additivity.

The following observation will allow restating SHNC in analytic terms.

Theorem 14 (Restating reduced rank.). If $\Gamma$ is a group, $\hat{Y}$ is a forest with a free cocompact $\Gamma$-action, and $Y$ is the (finite) quotient of $\hat{Y}$ by $\Gamma$, then

$$
\bar{r}(Y)=b_{1}^{(2)}(\hat{Y} ; \Gamma) .
$$

Proof. $\bar{r}(Y)$ and $b_{1}^{(2)}(\hat{Y} ; \Gamma)$ are additive under disjoint unions, so as in the proof of Lemma 13 we can assume that $Y$ is connected and $Y=\Gamma_{L} \backslash L$, where $L \in \operatorname{Comp}(\hat{Y})$ and $\Gamma_{L}:=\operatorname{Stab}_{\Gamma}(L)$. Then $L$ is a tree and $\Gamma_{L} \cong \pi_{1}(Y)$ acts on $L$ freely and cocompactly. If $L$ is infinite then $\Gamma_{L}$ is free and infinite, hence $-\chi(Y)=\operatorname{rk} \Gamma_{L}-1 \geq 0$. By Lemma 13(a),

$$
b_{1}^{(2)}(\hat{Y} ; \Gamma)=-\chi(Y)=\max \{0,-\chi(Y)\}=\bar{r}(Y) .
$$

If $L$ is finite, then $\Gamma_{L}=1$ and $Y \cong L$. Since $H_{1}(L)=0$, then by Lemma $13(\mathrm{~b})$,

$$
b_{1}^{(2)}(\hat{Y} ; \Gamma)=b_{1}(L)=\operatorname{dim} H_{1}(L)=0=\max \{0,-1\}=\max \{0,-\chi(L)\}=\bar{r}(Y) .
$$

5.5. Atomic decompositions of graphs. Let $\gamma: S \rightarrow X$ be an immersion of finite graphs, where $X$ is a connected finite graph with $\bar{r}(X)=1$, for example as in 3.6. Let $m:=\bar{r}(S)$, choose any maximal essential edge set $E=\left\{e_{1}, \ldots, e_{m}\right\}$ in $S$ (see 5.3 ), let $S_{i}^{\bullet}$ be a copy of $(S \backslash E) \cup\left\{e_{i}\right\}$, and define

$$
S^{\bullet}:=\bigsqcup_{i=1}^{m} S_{i}^{\bullet} .
$$

Let $\varphi_{i}: S_{i}^{\bullet} \hookrightarrow S$ be the inclusion map and $\varphi: S^{\bullet} \rightarrow S$ be induced by $\varphi_{i}$ termwise. 
Consider the maps $\psi_{i}:=\gamma \circ \varphi_{i}: S_{i}^{\bullet} \rightarrow X$. Let $\psi: S^{\bullet} \rightarrow X$ be induced by $\psi_{i}$. Then we have the commutative diagrams
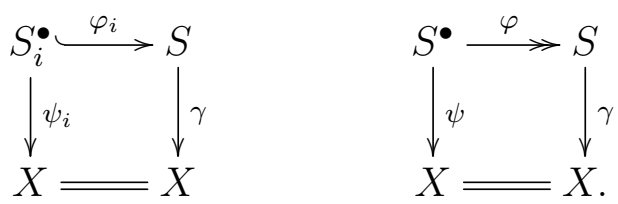

Define graphs $\hat{S}_{i}^{\bullet}, \hat{S}^{\bullet}$ and maps $\hat{\varphi}_{i}, \hat{\psi}_{i}, \hat{\varphi}, \hat{\psi}$ by pulling back the above diagrams by the universal cover map $\hat{X}_{\Delta} \rightarrow X$ as follows.
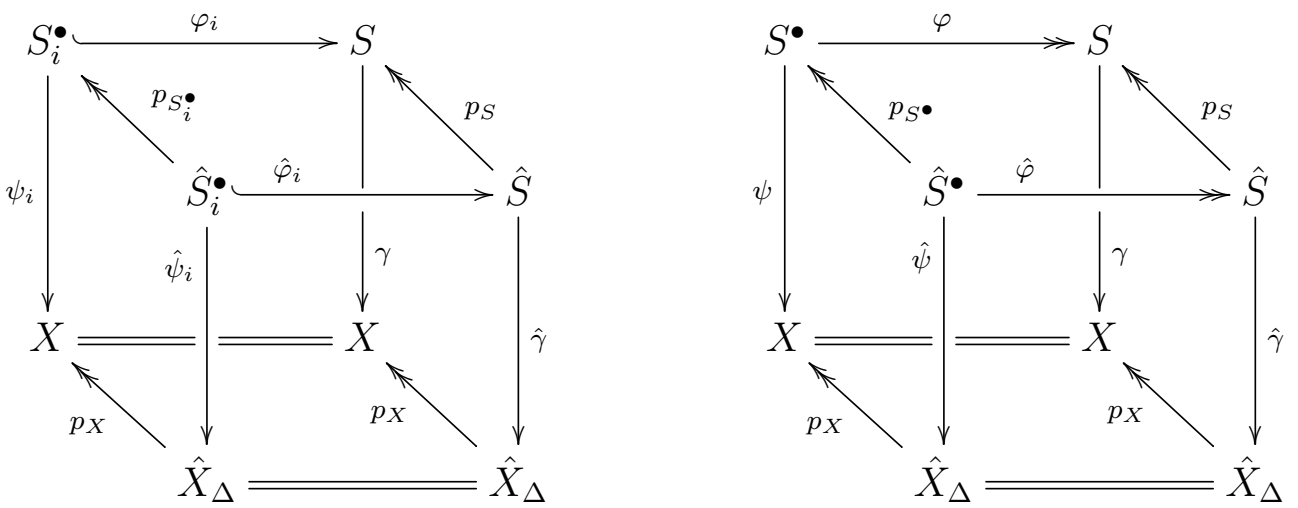

Let $\Delta$ be the diagonal subgroup in $\Gamma \times \Gamma$. The free $\Delta$-action on $\hat{X}_{\Delta}$ induces free $\Delta$-actions on $\hat{S}, \hat{S}_{i}^{\bullet}$, and $\hat{S}^{\bullet}$.

Define graphs $\hat{S}_{i}^{\bullet}, \hat{S} \bullet$ and maps $\hat{\hat{\varphi}}_{i}, \hat{\psi}_{i}, \hat{\hat{\varphi}}, \hat{\psi}$ by pulling back diagrams (16) by the composition $p_{X} \circ q_{X}: \hat{X} \rightarrow X$ as in (7).
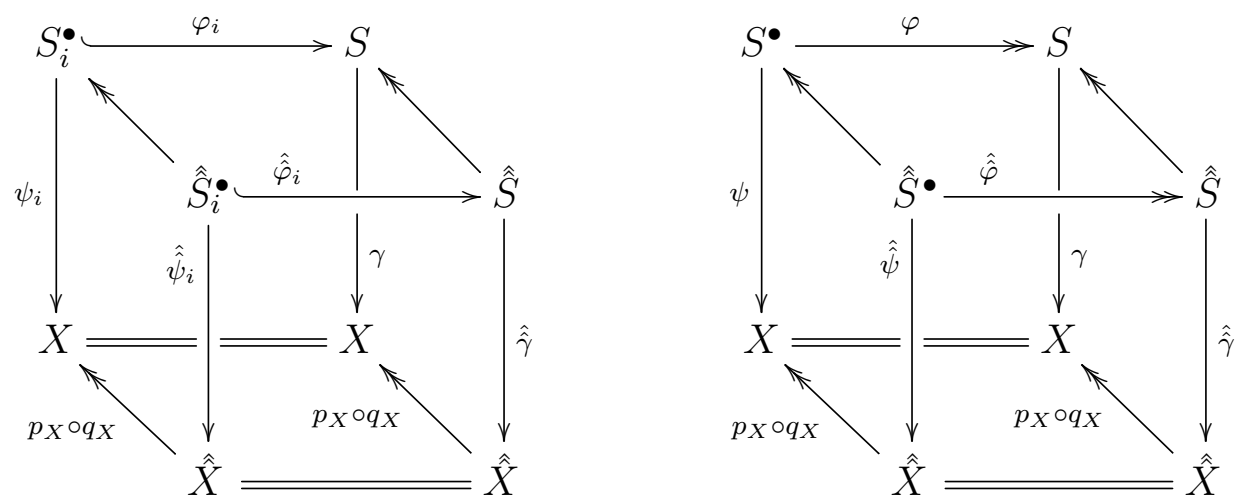

The free $\Gamma \times \Gamma$-action on $\hat{X}$ induces free $\Gamma \times \Gamma$-actions on $\hat{\hat{S}}, \hat{S}_{i}^{\bullet}$, and $\hat{S}^{\bullet}$.

Then $\hat{S}^{\bullet}=\bigsqcup_{i=1}^{m} \hat{S}_{i}^{\bullet}$. It seems reasonable to call the graphs $S_{i}^{\bullet}$ atoms of $S, S^{\bullet}$ an atomic decomposition of $S, \varphi: S^{\bullet} \rightarrow S$ an atomic map for $S$, and similarly for $\hat{S}$ and $\hat{S}$. 
The vertical and horizontal maps in the diagrams (17) are finite-to-one, so they induce chain maps on $\ell^{2}$-chains $C_{*}^{(2)}(\cdot)$ and on the $\ell^{2}$-homology $\mathrm{H}(\cdot)$.

For each $i$, define

$$
\begin{aligned}
& \hat{T}:=\hat{\varphi}\left(\hat{S}_{i}^{\bullet}\right) \cap \bigcup_{j \neq i} \hat{\varphi}\left(\hat{S}_{j}^{\bullet}\right) \subseteq \hat{S}, \\
& T:=\varphi\left(S_{i}^{\bullet}\right) \cap \bigcup_{j \neq i} \varphi\left(S_{j}^{\bullet}\right) \subseteq S .
\end{aligned}
$$

\section{Lemma 15.}

(a) $T$ is a garden.

(b) $\hat{T}$ is $\Delta$-invariant.

(c) $p_{S}^{-1}(T)=\hat{T}$.

(d) $\Delta \backslash \hat{T} \cong T$.

Proof. (a) By the definition of atoms $\hat{S}_{i}^{\bullet}$,

$$
T=\varphi\left(S_{i}^{\bullet}\right) \cap \bigcup_{j \neq i} \varphi\left(S_{j}^{\bullet}\right)=\left[(S \backslash E) \cup\left\{e_{i}\right\}\right] \cap \bigcup_{j \neq i}\left[(S \backslash E) \cup\left\{e_{j}\right\}\right]=S \backslash E .
$$

Since $E$ is a maximal essential set, $S \backslash E$ is a garden.

(b) The preimages of cells in $X$ under $p_{X}: \hat{X}_{\Delta} \rightarrow X$ are exactly $\Delta$-orbits. Since $\hat{S}_{i}^{\bullet}$ is the pull-back of $S_{i}^{\bullet}$, preimages of cells in $S_{i}^{\bullet}$ are exactly $\Delta$-orbits in $\hat{S}_{i}^{\bullet}$, so $\hat{S}_{i}^{\bullet}$ is $\Delta$-invariant.

(c) A subset $Q \subseteq \hat{S}$ is called $p_{S}$-saturated if $p_{S}^{-1}\left(p_{s}(Q)\right)=Q$. If $Q, Q^{\prime} \subseteq \hat{S}$ are $p_{S^{-} \text {saturated }}$ then $Q \cap Q^{\prime}$ and $Q \cup Q^{\prime}$ are $p_{S^{-} \text {-saturated and }}$

$$
p_{S}\left(Q \cap Q^{\prime}\right)=p_{S}(Q) \cap p_{S}\left(Q^{\prime}\right), \quad p_{S}\left(Q \cup Q^{\prime}\right)=p_{S}(Q) \cup p_{S}\left(Q^{\prime}\right) .
$$

The preimages of cells in $S$ under $p_{S}: \hat{S} \rightarrow S$ are exactly $\Delta$-orbits. Then $Q \subseteq \hat{S}$ is $p_{S^{-} \text {-saturated }}$ if and only if it is $\Delta$-invariant. $\hat{S}_{i}^{\bullet}$ is $\Delta$-invariant, hence $\hat{\varphi}\left(\hat{S}_{i}^{\bullet}\right)$ is $\Delta$-invariant, hence $\hat{\varphi}\left(\hat{S}_{i}^{\bullet}\right)$ is $p_{S^{-}}$-saturated, and any unions an intersections of such are $p_{S^{-} \text {-saturated. }}$

Since $p_{X}: \hat{X}_{\Delta} \rightarrow X$ is surjectitve then its pull-back $p_{S} \bullet: \hat{S}_{i}^{\bullet} \rightarrow S_{i}^{\bullet}$ is surjective for each $i$. Thus

$$
\begin{aligned}
& p_{S}^{-1}(T)=p_{S}^{-1}\left[\varphi\left(S_{i}^{\bullet}\right) \cap \bigcup_{j \neq i} \varphi\left(S_{j}^{\bullet}\right)\right]=p_{S}^{-1}\left[\varphi\left(p_{S}\left(\hat{S}_{i}^{\bullet}\right)\right) \cap \bigcup_{j \neq i} \varphi\left(p_{S} \bullet\left(\hat{S}_{j}^{\bullet}\right)\right)\right] \\
& =p_{S}^{-1}\left[p_{S}\left(\hat{\varphi}\left(\hat{S_{i}^{\bullet}}\right)\right) \cap \bigcup_{j \neq i} p_{S}\left(\hat{\varphi}\left(\hat{S}_{j}^{\bullet}\right)\right)\right]=p_{S}^{-1}\left[p_{S}\left(\hat{\varphi}\left(\hat{S}_{i}^{\bullet}\right) \cap \bigcup_{j \neq i} \hat{\varphi}\left(\hat{S}_{j}^{\bullet}\right)\right)\right] \\
& =p_{S}^{-1}\left(p_{S}(\hat{T})\right)=\hat{T} .
\end{aligned}
$$

(d) $p_{S}^{-1}(T)=T$ and preimages of cells in $S$ under $p_{S}: \hat{S} \rightarrow S$ are exactly $\Delta$-orbits, hence $\Delta \backslash \hat{T} \cong T$.

Theorem 16. Let $\Gamma:=\pi_{1}(X)$ and $\varphi: S^{\bullet} \rightarrow S$ and $\hat{\varphi}: \hat{S}^{\bullet} \rightarrow \hat{S}$ be atomic maps as above. Then

(a) for each $i, \bar{r}\left(S_{i}^{\bullet}\right)=1$, or equivalently, $\beta_{1}^{(2)}\left(\mathrm{H}\left(\hat{S}_{i}^{\bullet}\right), \Gamma\right)=1$, 
(b) the induced maps $\hat{\psi}_{i}: \mathrm{H}\left(\hat{S}_{i}^{\bullet}\right) \rightarrow \mathrm{H}(\hat{X})$ and $\hat{\varphi}: \mathrm{H}\left(\hat{S}^{\bullet}\right) \rightarrow \mathrm{H}(\hat{S})$ are weak isomorphisms of Hilbert $\Delta$-modules,

(c) the induced maps $\hat{\psi}_{i}: \mathrm{H}\left(\hat{S}_{i}^{\bullet}\right) \rightarrow \mathrm{H}(\hat{X})$ and $\hat{\hat{\varphi}}: \mathrm{H}(\hat{\hat{S}} \bullet) \rightarrow \mathrm{H}(\hat{\hat{S}})$ are weak isomorphisms of Hilbert $\Gamma \times \Gamma$-modules.

Proof. (a) Adding an edge to a finite graph increases reduced rank either by 0 or by 1 . Since $E$ is essential, adding each edge $e_{i} \in E$ to $S \backslash E$ must increase reduced rank by 1 , because otherwise adding $E$ to $S \backslash E$ would not increase reduced rank by \#E. Since $E$ is maximal, $\bar{r}(S \backslash E)=0$. It follows that $\bar{r}\left(S_{i}^{\bullet}\right)=1$.

(b) We have the induced diagrams on $\ell^{2}$-chains
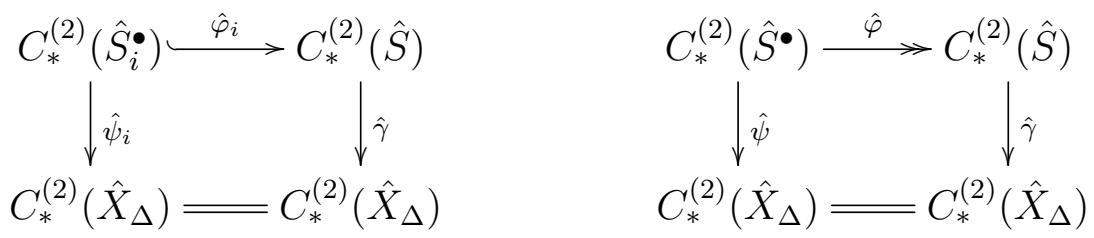

and on $\ell^{2}$-homology
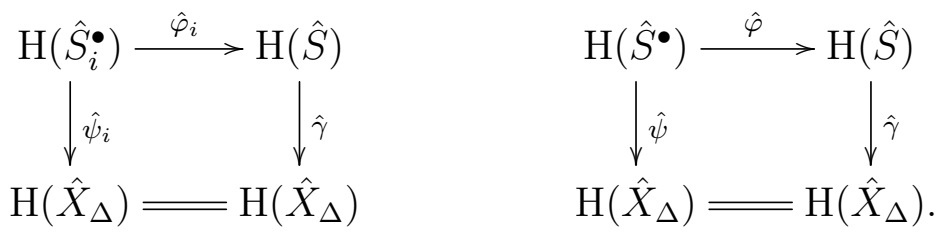

Since $\bar{r}\left(S_{i}^{\bullet}\right)=1=\bar{r}(X), \Delta \backslash \hat{S}_{i}^{\bullet} \cong S_{i}^{\bullet}$, and $\Delta \backslash \hat{X}_{\Delta} \cong X$, then by Theorem 14 ,

$$
\operatorname{dim}_{\Delta} \mathrm{H}\left(\hat{S}_{i}^{\bullet}\right)=\bar{r}\left(S_{i}^{\bullet}\right)=1=\bar{r}(X)=\operatorname{dim}_{\Delta} \mathrm{H}\left(\hat{X}_{\Delta}\right) .
$$

$$
\begin{aligned}
& \operatorname{Ker}\left[\hat{\psi}_{i}: \mathrm{H}\left(\hat{S}_{i}^{\bullet}\right) \rightarrow \mathrm{H}\left(\hat{X}_{\Delta}\right)\right] \\
& =\mathrm{H}\left(\hat{S}_{i}^{\bullet}\right) \cap \operatorname{Ker}\left[\hat{\psi}_{i}: C_{1}^{(2)}\left(\hat{S}_{i}^{\bullet}\right) \rightarrow C_{1}^{(2)}\left(\hat{X}_{\Delta}\right)\right] \\
& =\operatorname{Ker}\left[\partial: C_{1}^{(2)}\left(\hat{S}_{i}^{\bullet}\right) \rightarrow C_{0}^{(2)}\left(\hat{S}_{i}^{\bullet}\right)\right] \cap \operatorname{Ker}\left[\hat{\psi}_{i}: C_{1}^{(2)}\left(\hat{S}_{i}^{\bullet}\right) \rightarrow C_{1}^{(2)}\left(\hat{X}_{\Delta}\right)\right],
\end{aligned}
$$

hence by Theorem 10(1),

$$
\operatorname{dim}_{\Delta} \operatorname{Ker}\left[\hat{\psi}_{i}: \mathrm{H}\left(\hat{S}_{i}^{\bullet}\right) \rightarrow \mathrm{H}\left(\hat{X}_{\Delta}\right)\right] \in \mathbb{Z} .
$$

The map $\hat{\psi}_{i}: \mathrm{H}\left(\hat{S}_{i}^{\bullet}\right) \rightarrow \mathrm{H}\left(\hat{X}_{\Delta}\right)$ is not zero because components of $\hat{S}_{i}^{\bullet}$ are mapped injectively into $\hat{X}_{\Delta}$. Hence

$$
\operatorname{dim}_{\Delta} \operatorname{Ker}\left[\hat{\psi}_{i}: \mathrm{H}\left(\hat{S}_{i}^{\bullet}\right) \rightarrow \mathrm{H}\left(\hat{X}_{\Delta}\right)\right]=0,
$$

so $\hat{\psi}_{i}: \mathrm{H}\left(\hat{S}_{i}^{\bullet}\right) \rightarrow \mathrm{H}\left(\hat{X}_{\Delta}\right)$ is injective, and therefore is a weak isomorphism.

The same argument shows that $\hat{\varphi}_{i}: \mathrm{H}\left(\hat{S}_{i}^{\bullet}\right) \rightarrow \mathrm{H}(\hat{S})$ is injective for each $i$. Since

$$
\operatorname{dim}_{\Delta} \mathrm{H}\left(\hat{S}^{\bullet}\right)=\sum_{i=1}^{m} \operatorname{dim}_{\Delta} \mathrm{H}\left(\hat{S}_{i}^{\bullet}\right)=\sum_{i=1}^{m} 1=m=\bar{r}(S)=\operatorname{dim}_{\Delta} \mathrm{H}(\hat{S}),
$$


it only remains to show that the map

$$
\hat{\varphi}: \mathrm{H}\left(\hat{S}^{\bullet}\right)=\mathrm{H}\left(\bigsqcup_{i=1}^{m} \hat{S}_{i}^{\bullet}\right)=\bigoplus_{i=1}^{m} \mathrm{H}\left(\hat{S}_{i}^{\bullet}\right) \rightarrow \mathrm{H}(\hat{S})
$$

is injective. This is equivalent to saying that for each $i$,

$$
\hat{\varphi}\left(\mathrm{H}\left(\hat{S}_{i}^{\bullet}\right)\right) \cap \sum_{j \neq i} \hat{\varphi}\left(\mathrm{H}\left(\hat{S}_{j}^{\bullet}\right)\right)=0 .
$$

$\hat{\varphi}_{i}$ is a pull-back of the inclusion map $\varphi_{i}$, so it is injective, i.e. $\hat{\varphi}_{i}: \hat{S}_{i}^{\bullet} \rightarrow \hat{\varphi}_{i}\left(\hat{S}_{i}^{\bullet}\right)$ is an isomorphism. Then $\hat{\varphi}\left(\mathrm{H}\left(\hat{S}_{i}^{\bullet}\right)\right)=\mathrm{H}\left(\hat{\varphi}\left(\hat{S}_{i}^{\bullet}\right)\right)$, and $(21)$ is equivalent to

$$
\mathrm{H}\left(\hat{\varphi}\left(\hat{S}_{i}^{\bullet}\right)\right) \cap \sum_{j \neq i} \mathrm{H}\left(\hat{\varphi}\left(\hat{S}_{j}^{\bullet}\right)\right)=0 .
$$

Since $\Delta \backslash \hat{T} \cong T$ and $T$ is a garden by Lemma 15 , we have

$$
\operatorname{dim}_{\Delta} \mathrm{H}(\hat{T})=\bar{r}(T)=0
$$

Then

$$
\begin{aligned}
& \mathrm{H}\left(\hat{\varphi}\left(\hat{S}_{i}^{\bullet}\right)\right) \cap \sum_{j \neq i} \mathrm{H}\left(\hat{\varphi}\left(\hat{S}_{j}^{\bullet}\right)\right)=\mathrm{H}\left(\hat{\varphi}\left(\hat{S}_{i}^{\bullet}\right)\right) \cap \mathrm{H}\left(\bigcup_{j \neq i} \hat{\varphi}\left(\hat{S}_{j}^{\bullet}\right)\right) \\
& =\mathrm{H}\left(\hat{\varphi}\left(\hat{S}_{i}^{\bullet}\right) \cap \bigcup_{j \neq i} \hat{\varphi}\left(\hat{S}_{j}^{\bullet}\right)\right)=\mathrm{H}(\hat{T})=0,
\end{aligned}
$$

which proves $(21)$.

(c) By Lemma $9, \hat{X}=\operatorname{Ind}_{\Delta}^{\Gamma \times \Gamma} \hat{X}_{\Delta}$, hence the diagrams

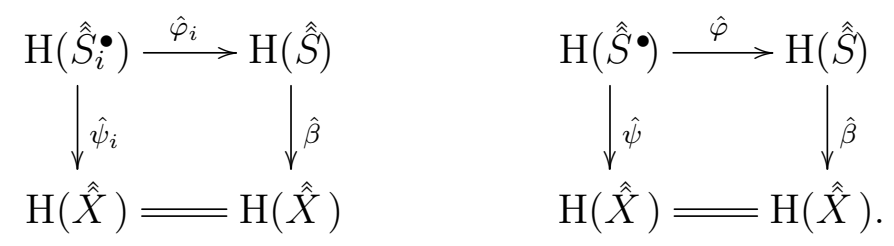

are obtained by applying $\operatorname{Ind}_{\Delta}^{\Gamma \times \Gamma}$ to diagrams (20). Induction preserves weak equivalence.

\section{Change of subgroups.}

We describe a procedure that changes the stabilizers of components in $\hat{Y}^{\prime}$ and $\hat{Z}^{\prime}$. Eventually we will make all the stabilizers the same; this will be needed later to define arrangements.

\subsection{Induced systems.}

Lemma 17. Suppose

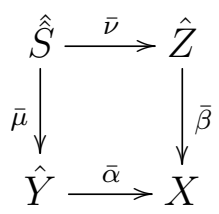


is part of a system $\mathcal{S}$ as in (5) and $\Gamma_{1}$ and $\Gamma_{2}$ are arbitrary subgroups of $\Gamma$. Denote

$$
Y_{\sharp}:=\Gamma_{1} \backslash \hat{Y}, \quad Z_{\sharp}:=\Gamma_{2} \backslash \hat{Z}, \quad S_{\sharp}:=\left(\Gamma_{1} \times \Gamma_{2}\right) \backslash \hat{S} .
$$

Then the quotient maps $q_{Y_{\sharp}}: \hat{Y} \rightarrow Y_{\sharp}, q_{Z_{\sharp}}: \hat{Z} \rightarrow Z_{\sharp}$, and $q_{S_{\sharp}}: \hat{S} \rightarrow S_{\sharp}$ fit in a commutative diagram

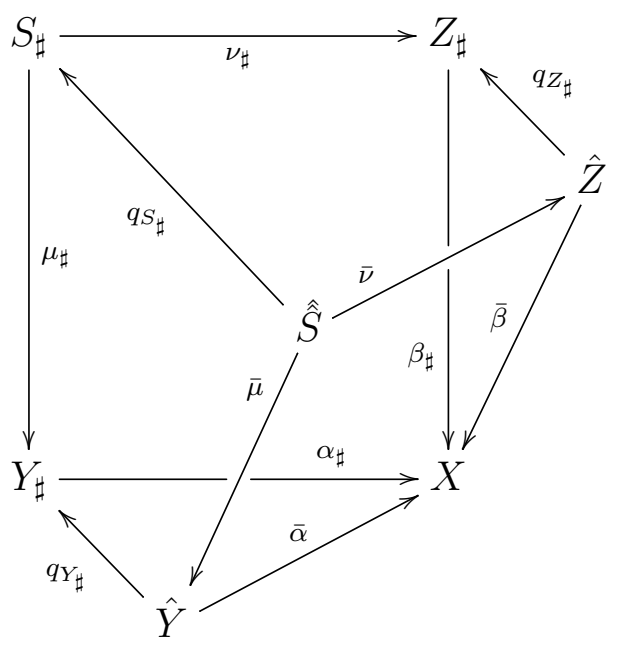

in which the two squares $\left(X, Y_{\sharp}, Z_{\sharp}, S_{\sharp}\right)$ and $(X, \hat{Y}, \hat{Z}, \hat{S})$ are pull-back diagrams.

Proof. Diagram (23) is a pull-back diagram being part of a system. It remains to check that the other square in (24) is a pull-back diagram. The preimage of a cell $\sigma$ in $X$ under $\bar{\alpha}$ can be identified with $\alpha^{-1}(\sigma) \times \Gamma$, its preimage under $\bar{\beta}$ with $\beta^{-1}(\sigma) \times \Gamma$, and its preimage under $\bar{\alpha} \circ \bar{\mu}$ with $\alpha^{-1}(\sigma) \times \beta^{-1}(\sigma) \times \Gamma \times \Gamma$, which is the product of the other two. The restrictions of the quotient maps $q_{Y_{\sharp}}, q_{Z_{\sharp}}, q_{Z_{\sharp}}$ to these preimages can be identified with the quotient maps

$$
\begin{aligned}
& \alpha^{-1}(\sigma) \times \Gamma \rightarrow \alpha^{-1}(\sigma) \times\left(\Gamma / \Gamma_{1}\right), \\
& \beta^{-1}(\sigma) \times \Gamma \rightarrow \beta^{-1}(\sigma) \times\left(\Gamma / \Gamma_{2}\right), \\
& \alpha^{-1}(\sigma) \times \beta^{-1}(\sigma) \times \Gamma \times \Gamma \rightarrow \alpha^{-1}(\sigma) \times \beta^{-1}(\sigma) \times\left((\Gamma \times \Gamma) /\left(\Gamma_{1} \times \Gamma_{2}\right)\right) .
\end{aligned}
$$

Now it remains to note that the fiber product structure is preserved by the quotients: $\alpha^{-1}(\sigma) \times$ $\beta^{-1}(\sigma) \times\left((\Gamma \times \Gamma) /\left(\Gamma_{1} \times \Gamma_{2}\right)\right)$ is the product of $\alpha^{-1}(\sigma) \times\left(\Gamma / \Gamma_{1}\right)$ and $\beta^{-1}(\sigma) \times\left(\Gamma / \Gamma_{2}\right)$.

Let $\left(\mathcal{S}^{\prime}, \mathcal{S}\right)$ be a pair of systems as in 3.4 and $G$ be some subgroup of $\Gamma$. We will be particularly interested in applying Lemma 17 to these two systems in the case when $\Gamma_{1}:=G$ and $\Gamma_{2}:=G$.

Recall that $Z \cong \Gamma \backslash \hat{Z}$ and $Z^{\prime} \cong \Gamma \backslash \hat{Z}^{\prime}$, and $p_{Z}: \hat{Z} \rightarrow Z$ and $p_{Z}: \hat{Z}^{\prime} \rightarrow Z^{\prime}$ are the quotient maps. Denote

$$
\begin{aligned}
& Y_{G}:=G \backslash \hat{Y}, \quad Z_{G}:=G \backslash \hat{Z}, \quad S_{G}:=(G \times G) \backslash \hat{S}, \\
& Y_{G}^{\prime}:=G \backslash \hat{Y}, \quad Z_{G}^{\prime}:=G \backslash \hat{Z}, \quad S_{G}^{\prime}:=(G \times G) \backslash \hat{S}^{\prime},
\end{aligned}
$$

and let $q_{Y_{G}}: \hat{Y} \rightarrow Y_{G}, q_{S_{G}}: \hat{S} \rightarrow S_{G}, q_{Y_{G}}: \hat{Y} \rightarrow Y_{G}^{\prime}$, and $q_{S_{G}}: \hat{S}^{\prime} \rightarrow S_{G}^{\prime}$ be the corresponding quotient maps. By Lemma 17 they fit in the two commutative diagrams 


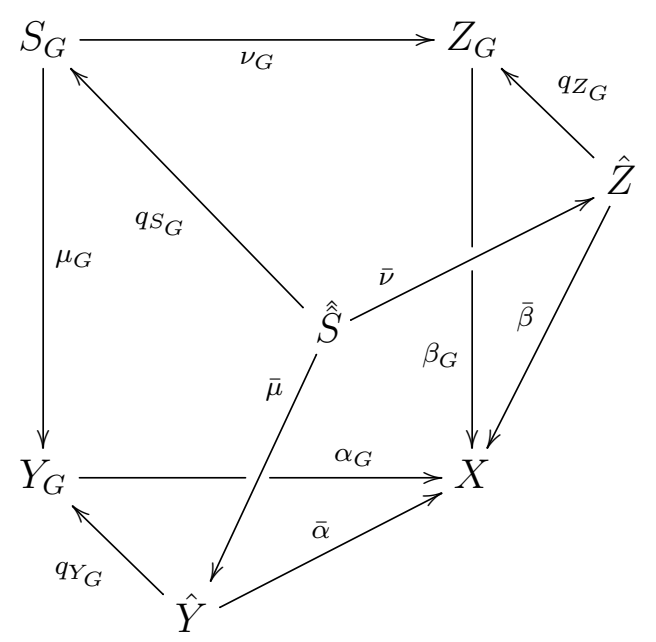

$(26)$

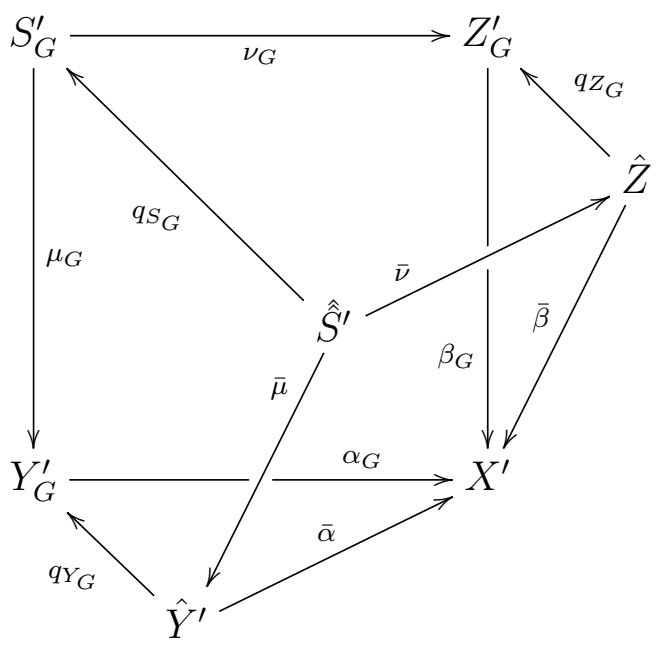


whose squares $\left(X, Y_{G}, Z_{G}, S_{G}\right)$ and $\left(X, Y_{G}^{\prime}, Z_{G}^{\prime}, S_{G}^{\prime}\right)$ are pull-back diagrams. Hence the maps $\alpha_{G}, \beta_{G}$ from (25) and the quotient map $p_{X}: \hat{X} \rightarrow X$ generate another system

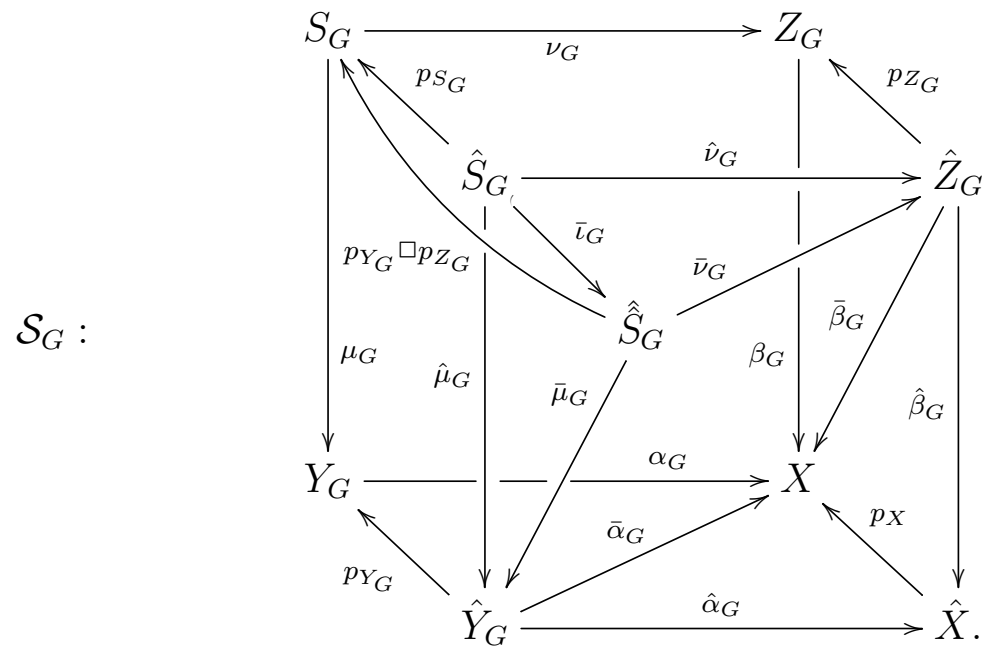

This defines complexes $\hat{Y}_{G}, \hat{Z}_{G}, \hat{S}_{G}, \hat{S}_{G}$, and the maps to and from them. $\mathcal{S}_{G}$ is a $\Gamma$-system, and will be called the system induced by $\mathcal{S}$ from $\Gamma$ to $G$.

Similarly, the maps $\alpha_{G}$ and $\beta_{G}$ from (26) and $p_{X}$ generate the system

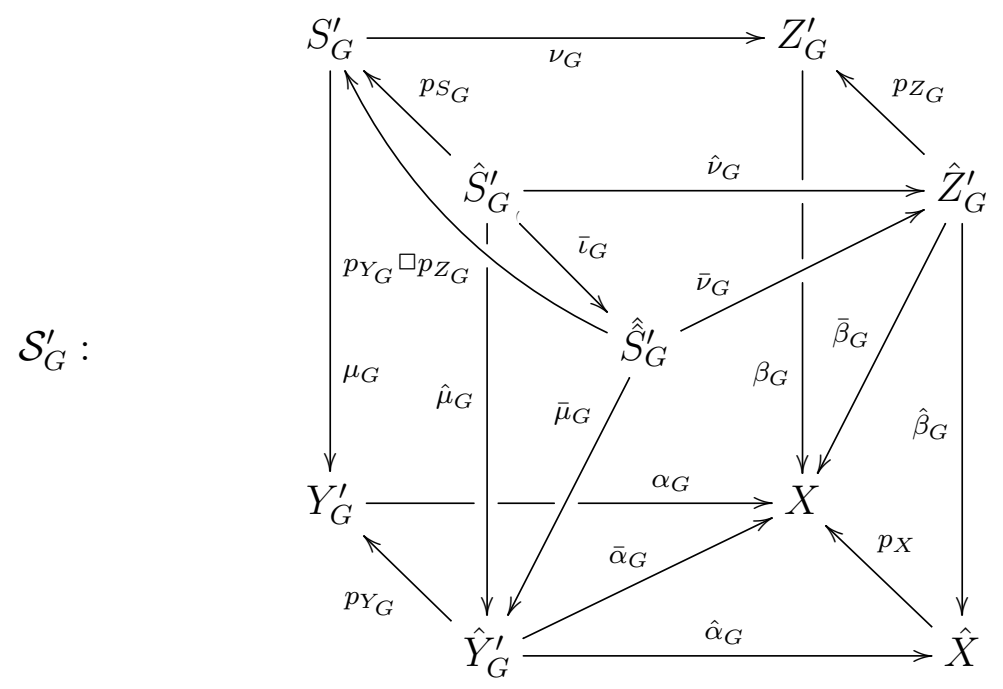

called the system induced by $\mathcal{S}^{\prime}$ from $\Gamma$ to $G$. This defines complexes $\hat{Y}_{G}^{\prime}, \hat{Z}_{G}^{\prime}, \hat{S}_{G}^{\prime}$, $\hat{S}_{G}^{\prime}$, and the maps to and from them. $\mathcal{S}_{G}$ is a subsystem of $\mathcal{S}_{G}^{\prime}$.

6.2. Symmetric systems. We will show that it suffices to check SHNC only for graph systems of certain special type.

Definition 18. Assume that $\left(\mathcal{S}^{\prime}, \mathcal{S}\right)$ is a pair of systems as in 3.4. The system $\mathcal{S}^{\prime}$ will be called symmetric if it has the following additional properties:

- $Y^{\prime} \cong Z^{\prime}$ 
- the covers $\alpha: Y^{\prime} \rightarrow X$ and $\beta: Z^{\prime} \rightarrow X$ are isomorphic, and

- there exists is a normal subgroup $G$ in $\Gamma$ such that $\operatorname{Stab}_{\Gamma}\left(K^{\prime}\right)=G$ for each $K^{\prime} \in$ $\operatorname{Comp}\left(\hat{Y}^{\prime}\right)$ and for each $K^{\prime} \in \operatorname{Comp}\left(\hat{Z}^{\prime}\right)$.

A pair of systems $\left(\mathcal{S}^{\prime}, \mathcal{S}\right)$ will be called symmetric if $\mathcal{S}^{\prime}$ is symmetric. A pair of systems $\left(\mathcal{S}^{\prime}, \mathcal{S}\right)$ will be called connected if both $\mathcal{S}$ and $\mathcal{S}^{\prime}$ are connected, that is if $X, Y, Z, Y^{\prime}$, and $Z^{\prime}$ are connected.

In particular, the above covers $\alpha: Y^{\prime} \rightarrow X$ and $\beta: Z^{\prime} \rightarrow X$ are the regular covers corresponding to the normal subgroup $G$ of $\Gamma$. Denote $\bar{\Gamma}:=\Gamma / G$.

Lemma 19. Let $Y^{\prime}$ be part of a connected symmetric system $\mathcal{S}^{\prime}$. Then the induced $\bar{\Gamma}$-action on $\operatorname{Comp}\left(Y^{\prime}\right)$ is free and transitive.

Proof. Freeness follows from Theorem $7(\mathrm{~d})$ and transitivity from Theorem $7(\mathrm{e})$.

\section{Restatements of SHNC.}

In this section we provide several equivalent restatements of SHNC.

Lemma 20. For any graph system $\mathcal{S}$ as in 3.6,

$$
b_{1}^{(2)}(\hat{S} ; \Gamma \times \Gamma)=b_{1}^{(2)}(\hat{S} ; \Delta) .
$$

For a proof, one can use Theorem 14, and the isomorphisms $(\Gamma \times \Gamma) \backslash \hat{S} \cong S$ and $\Gamma \backslash \hat{S} \cong S$ to deduce that both $b_{1}^{(2)}(\hat{S} ; \Gamma \times \Gamma)$ and $b_{1}^{(2)}(\hat{S} ; \Gamma)$ equal $\bar{r}(S)$. We emphasize that there is the following direct analytic proof that does not use reduced rank.

Proof. By Lemma 9, $\hat{S}=\operatorname{Ind}_{\Delta}^{\Gamma \times \Gamma} \hat{S}$, hence

$$
b_{1}^{(2)}(\hat{S} ; \Gamma \times \Gamma)=\operatorname{dim}_{\Gamma \times \Gamma} \mathrm{H}(\hat{\hat{S}})=\operatorname{dim}_{\Delta} \mathrm{H}(\hat{S})=b_{1}^{(2)}(\hat{S} ; \Delta) .
$$

Lemma 21. The map

$$
\Phi: \operatorname{Comp}(\hat{Y}) \times \operatorname{Comp}(\hat{Z}) \rightarrow \operatorname{Comp}(\hat{S})
$$

defined by $\Phi(K, L):=K \hat{\square} L \subseteq \hat{S}$ is a bijection.

Proof. Since the restricted maps $\hat{\alpha}: K \rightarrow \hat{X}$ and $\hat{\beta}: L \rightarrow \hat{X}$ are injective, $K \hat{\square} L$ is isomorphic to $\hat{\alpha}(K) \cap \hat{\beta}(L)$. Since $\hat{\alpha}(K)$ and $\hat{\beta}(L)$ are convex in the tree $\hat{X}$, then so is $\hat{\alpha}(K) \cap \hat{\beta}(L)$. Then $K \hat{\square} L$ is a connected component of $\hat{S}$, so $\Phi$ is well-defined. The rest is clear.

Let $A \backslash \Gamma / B$ be the set of all double cosets $A g B$ for $g \in \Gamma$ and $s: A \backslash \Gamma / B \rightarrow \Gamma$ be a section of the quotient map $\Gamma \rightarrow A \backslash \Gamma / B$. Then $s(A \backslash \Gamma / B)$ is a set of representatives of double cosets.

We will use the notations

$$
A^{z}:=z^{-1} A z, \quad{ }^{z} B:=z B z^{-1} .
$$

For $z \in \Gamma$, let $t_{z}: \Gamma /\left(A \cap{ }^{z} B\right) \rightarrow \Gamma$ be a set-theoretic section of the quotient $\Gamma \rightarrow \Gamma /\left(A \cap{ }^{z} B\right)$, then $t_{z}\left(\Gamma /\left(A \cap{ }^{z} B\right)\right)$ is a set of representatives for the left cosets of $A \cap{ }^{z} B$ in $\Gamma$. 
Denote

$$
T(A, B):=\left\{(y, z) \mid z \in s(A \backslash \Gamma / B), y \in t_{z}\left(\Gamma /\left(A \cap{ }^{z} B\right)\right)\right\} \subseteq \Gamma \times \Gamma .
$$

Choose $K \in \operatorname{Comp}(\hat{Y})$ and $L \in \operatorname{Comp}(\hat{Z})$ such that $\operatorname{Stab}_{\Gamma}(K)=A$ and $\operatorname{Stab}_{\Gamma}(L)=B$.

Lemma 22. For a connected graph system $\mathcal{S}$, the map

$$
T(A, B) \rightarrow \operatorname{Comp}(\hat{S}), \quad(y, z) \mapsto y K \hat{\square} y z L \subseteq \hat{S}
$$

is a bijection.

Proof. Injectivity.

$$
\begin{aligned}
& y K \hat{\square} y z L=y^{\prime} K \hat{\square} y^{\prime} z^{\prime} L \quad \Rightarrow \quad K \hat{\square} L=\left(y^{-1} y^{\prime} K\right) \hat{\square}\left(z^{-1} y^{-1} y^{\prime} z^{\prime} L\right) \Rightarrow \\
& y^{-1} y^{\prime} \in \operatorname{Stab}_{\Gamma}(K)=A \quad \text { and } \quad z^{-1} y^{-1} y^{\prime} z^{\prime} \in \operatorname{Stab}_{\Gamma}(L)=B \Rightarrow \\
& z \in A z^{\prime} B \Rightarrow z=z^{\prime} \Rightarrow z^{-1}\left(y^{-1} y^{\prime}\right) z \in B \Rightarrow \\
& y^{-1} y^{\prime} \in A \cap{ }^{z} B \Rightarrow y\left(A \cap{ }^{z} B\right)=y^{\prime}\left(A \cap{ }^{z} B\right) \Rightarrow y=y^{\prime} .
\end{aligned}
$$

Surjectivity. Each component of $\hat{S}$ has the form $K^{\prime} \hat{\square} L^{\prime}$ for some $K^{\prime} \in \operatorname{Comp}(\hat{Y})$ and $L^{\prime} \in$ $\operatorname{Comp}(\hat{Z})$. Since $Y$ and $Z$ are connected, then the $\Gamma$-actions on $\operatorname{Comp}(\hat{Y})$ and on $\operatorname{Comp}(\hat{Z})$ are transitive. Then $K^{\prime}=u K$ and $L^{\prime}=v L$ for some $u, v \in \Gamma$.

Let $z:=s\left(A\left(u^{-1} V\right) B\right)$, then $z=a u^{-1} v b$ for some $a \in A, b \in B$. Let $y:=t_{z}\left(u a^{-1}\left(A \cap{ }^{z} B\right)\right)$, then $y=u a^{-1} c$ for some $c \in A \cap{ }^{z} B=\operatorname{Stab}_{\Gamma}(K) \cap \operatorname{Stab}_{\Gamma}(z L)$. We have

$$
\begin{aligned}
& c K=K, \quad c z L=z L, \quad a^{-1} K=K, \quad b L=L, \\
& \Phi(y, z)=y K \hat{\square} y z L=u a^{-1} c K \hat{\square} u a^{-1} c z L \\
& =u a^{-1} K \hat{\square} u a^{-1} z L=u a^{-1} K \hat{\square} v b L=u K \hat{\square} v L .
\end{aligned}
$$

Theorem 23. Each of the following statements is equivalent to SHNC.

(a) For any graph system $\mathcal{S}$ as in 3.6,

$$
b_{1}^{(2)}(\hat{S} ; \Gamma \times \Gamma) \leq b_{1}^{(2)}(\hat{Y} ; \Gamma) \cdot b_{1}^{(2)}(\hat{Z} ; \Gamma) .
$$

$\left(a^{\prime}\right)$ For any graph system $\mathcal{S}$ as in 3.6,

$$
b_{1}^{(2)}(\hat{S} ; \Gamma) \leq b_{1}^{(2)}(\hat{Y} ; \Gamma) \cdot b_{1}^{(2)}(\hat{Z} ; \Gamma) .
$$

(b) For any connected graph system $\mathcal{S}$ as in 3.7,

$$
b_{1}^{(2)}(\hat{S} ; \Gamma \times \Gamma) \leq b_{1}^{(2)}(\hat{Y} ; \Gamma) \cdot b_{1}^{(2)}(\hat{Z} ; \Gamma) .
$$

$\left(b^{\prime}\right)$ For any connected graph system $\mathcal{S}$ as in 3.7,

$$
b_{1}^{(2)}(\hat{S} ; \Gamma) \leq b_{1}^{(2)}(\hat{Y} ; \Gamma) \cdot b_{1}^{(2)}(\hat{Z} ; \Gamma) .
$$

(c) For any connected symmetric pair of graph systems $\left(\mathcal{S}^{\circ}, \mathcal{S}\right)$ as in 3.7 and 6.2,

$$
b_{1}^{(2)}(\hat{S} ; \Gamma \times \Gamma) \leq b_{1}^{(2)}(\hat{Y} ; \Gamma) \cdot b_{1}^{(2)}(\hat{Z} ; \Gamma) .
$$


$\left(c^{\prime}\right)$ For any connected symmetric pair of graph systems $\left(\mathcal{S}^{\circ}, \mathcal{S}\right)$ as in 3.7 and 6.2,

$$
b_{1}^{(2)}(\hat{S} ; \Gamma) \leq b_{1}^{(2)}(\hat{Y} ; \Gamma) \cdot b_{1}^{(2)}(\hat{Z} ; \Gamma) .
$$

Proof. Lemma 20 implies equivalences $(a) \Leftrightarrow\left(a^{\prime}\right),(b) \Leftrightarrow\left(b^{\prime}\right),(c) \Leftrightarrow\left(c^{\prime}\right)$. The implications $(a) \Rightarrow(b) \Rightarrow(c)$ are obvious.

$S H N C \Leftrightarrow\left(b^{\prime}\right)$. The assumptions of SHNC determine a connected graph system and vice versa. Choose $K \in \operatorname{Comp}(\hat{Y})$ and $L \in \operatorname{Comp}(\hat{Z})$ such that $\operatorname{Stab}_{\Gamma}(K)=A$ and $\operatorname{Stab}_{\Gamma}(L)=B$. Using Lemma 22 and induction,

$$
\begin{aligned}
& b_{1}^{(2)}(\hat{S} ; \Gamma)=\operatorname{dim}_{\Gamma} \mathrm{H}(\hat{S} ; \Gamma)=\operatorname{dim}_{\Gamma} \mathrm{H}\left(\bigsqcup_{M \in \operatorname{Comp}(\hat{S})} M\right) \\
& =\operatorname{dim}_{\Gamma} \mathrm{H}\left(\bigsqcup_{z \in s(A \backslash \Gamma / B)} \bigsqcup_{y \in t_{z}\left(\Gamma /\left(A \cap^{z} B\right)\right)} y K \hat{\square} y z L\right) \\
& =\sum_{z \in s(A \backslash \Gamma / B)} \bigoplus_{y \in t_{z}\left(\Gamma /\left(A \cap^{z} B\right)\right)} \mathrm{H}(y K \hat{\square} y z L) \\
& =\sum_{z \in s(A \backslash \Gamma / B)} \operatorname{dim}_{\Gamma} \operatorname{Ind}_{A \cap^{z} B} \mathrm{H}(K \hat{\square} z L) \\
& =\sum_{z \in s(A \backslash \Gamma / B)} \operatorname{dim}_{A \cap^{z} B} \mathrm{H}(K \hat{\square} z L) \\
& =\sum_{z \in s(A \backslash \Gamma / B)} b_{1}^{(2)}\left(K \hat{\square} z L ; A \cap{ }^{z} B\right) .
\end{aligned}
$$

By Lemma 21, each $K \hat{\square} z L$ is connected. Since it is isomorphic to a subset of the tree $\hat{X}$, each $K \hat{\square} z L$ is a tree. $A \cap{ }^{z} B$ is the stabilizer of $K \hat{\square} z L \subseteq \hat{S}$, and the quotient

$$
\left(A \cap{ }^{z} B\right) \backslash(K \hat{\square} z L) \cong \Gamma \backslash\left(\bigsqcup_{y \in t_{z}\left(\Gamma /\left(A \cap^{z} B\right)\right)} y K \hat{\square} y z L\right)
$$

is isomorphic to one of the components of $S$, so in particular it is finite. Then by Theorem 14,

$$
b_{1}^{(2)}\left(K \hat{\square} z L ; A \cap{ }^{z} B\right)=\bar{r}\left(A \cap{ }^{z} B\right) .
$$

This proves

$$
b_{1}^{(2)}(\hat{S} ; \Gamma)=\sum_{z \in s(A \backslash \Gamma / B)} \bar{r}\left(A \cap{ }^{z} B\right)=\sum_{z \in s(A \backslash \Gamma / B)} \bar{r}\left(A^{z} \cap B\right) .
$$

The last equality holds because $A \cap{ }^{z} B$ and $A^{z} \cap B$ are conjugates of each other. Theorem 14 also implies

$$
b_{1}^{(2)}(\hat{Y} ; A)=\bar{r}(A), \quad b_{1}^{(2)}(\hat{Z} ; B)=\bar{r}(B)
$$


The above three equalities show the equivalence of the inequalities

$$
\begin{aligned}
& b_{1}^{(2)}(\hat{S} ; \Gamma) \leq b_{1}^{(2)}(\hat{Y} ; \Gamma) \cdot b_{1}^{(2)}(\hat{Z} ; \Gamma) \quad \text { and } \\
& \sum_{z \in s(A \backslash \Gamma / B)} \bar{r}\left(A \cap{ }^{z} B\right) \leq \bar{r}(A) \cdot \bar{r}(B) .
\end{aligned}
$$

$(c) \Rightarrow(a)$. Consider any graph system $\mathcal{S}$ as in 3.6. Then it can be extended to a larger system $\mathcal{S}^{\circ}$ as in 3.7 in which

- $\alpha$ and $\beta$ are finite covers,

- the components of $\hat{Y}^{\circ}$ and $\hat{Z}^{\circ}$ map isomorphically onto $\hat{X}$,

- the stabilizers of the components of $\hat{Y}^{\circ}$ are conjugates of some subgroup $A^{\circ}$ in $\Gamma$ of finite index, and

- the stabilizers of the components of $\hat{Z}^{\circ}$ are conjugates of some subgroup $B^{\circ}$ in $\Gamma$ of finite index.

The $\Gamma$-actions on $\hat{Y}^{\circ}$ and $\hat{Z}^{\circ}$ induce a $\Gamma$-action on the finite set $\operatorname{Comp}\left(\hat{Y}^{\circ}\right) \sqcup \operatorname{Comp}\left(\hat{Z}^{\circ}\right)$, i.e. a group homomorphism $\Gamma \rightarrow \operatorname{Perm}\left[\operatorname{Comp}\left(\hat{Y}^{\circ}\right) \sqcup \operatorname{Comp}\left(\hat{Z}^{\circ}\right)\right]$, where Perm $[S]$ denotes the group of permutations of a set $S$. Denote

$$
G:=\operatorname{Ker}\left(\Gamma \rightarrow \operatorname{Perm}\left[\operatorname{Comp}\left(\hat{Y}^{\circ}\right) \sqcup \operatorname{Comp}\left(\hat{Z}^{\circ}\right)\right]\right)
$$

$G$ is a normal subgroup of finite index in $\Gamma$ which stabilizes each component in $\hat{Y}^{\circ}$ and $\hat{Z}^{\circ}$. Consider the pair of systems $\left(\mathcal{S}_{G}^{\circ}, \mathcal{S}_{G}\right)$ induced by the systems $\left(\mathcal{S}^{\circ}, \mathcal{S}\right)$ from $\Gamma$ to $G$ as in 6.1 . Since $Y_{G}^{\circ}=G \backslash \hat{Y}^{\circ}, Z_{G}^{\circ}=G \backslash \hat{Z}^{\circ}$, and the components of $\hat{Y}^{\circ}$ and $\hat{Z}^{\circ}$ are copies of $\hat{X}$, then each component of $Y_{G}^{\circ}$ and $Z_{G}^{\circ}$ is isomorphic to the quotient graph $G \backslash \hat{X}$. The components of $Y_{G}^{\circ}$ one-to-one correspond to the components of $\hat{Y}_{G}^{\circ}$ via the quotient map $q_{Y_{G}}$, and similarly for $Z_{G}^{\circ}$ and $\hat{Z}_{G}^{\circ}$.

First we claim that for this induced pair $\left(\mathcal{S}_{G}^{\circ}, \mathcal{S}_{G}\right)$ the inequality

$$
b_{1}^{(2)}\left(\hat{S}_{G} ; \Gamma \times \Gamma\right) \leq b_{1}^{(2)}\left(\hat{Y}_{G} ; \Gamma\right) \cdot b_{1}^{(2)}\left(\hat{Z}_{G} ; \Gamma\right)
$$

holds. Replace $Y_{G}$ with one of its components $K$, and $Z_{G}$ with one if its components $L$, and use them to generate a system $\mathcal{T}_{K, L}$ as in (27). $\mathcal{T}_{K, L}$ is a connected subsystem of $\mathcal{S}_{G}$. Next replace $Y_{G}^{\circ}$ with its component $K^{\circ}$ containing $K$, and $Z_{G}^{\circ}$ with its component $L^{\circ}$ containing $L$, and use them to generate a system $\mathcal{T}_{K, L}^{\circ}$ as in (28). $\mathcal{T}_{K, L}^{\circ}$ is a connected subsystem of $\mathcal{S}_{G}^{\circ}$. Since $K^{\circ}$ and $L^{\circ}$ are isomorphic, $\left(\mathcal{T}_{K, L}, \mathcal{T}_{K, L}^{\circ}\right)$ is a connected symmetric pair, so the above inequality holds for the system $\mathcal{T}_{K, L}$ by the assumptions of (c). Sum up all such inequalities over all pairs $(K, L) \in \operatorname{Comp}\left(Y_{G}\right) \times \operatorname{Comp}\left(Z_{G}\right)$ to obtain (29) for system $\mathcal{S}_{G}$. 
It remains to show that inequality $(29)$ for $\mathcal{S}_{G}$ implies the same inequality for the original system $\mathcal{S}$. This is done by tracing the definition of induced system:

$$
\begin{aligned}
& b_{1}^{(2)}\left(\hat{\hat{S}}_{G} ; \Gamma \times \Gamma\right) \leq b_{1}^{(2)}\left(\hat{Y}_{G} ; \Gamma\right) \cdot b_{1}^{(2)}\left(\hat{Z}_{G} ; \Gamma\right) \quad \Leftrightarrow \\
& \bar{r}\left(S_{G}\right) \leq \bar{r}\left(Y_{G}\right) \cdot \bar{r}\left(Z_{G}\right) \Leftrightarrow \\
& b_{1}^{(2)}(\hat{\hat{S}} ; G \times G) \leq b_{1}^{(2)}(\hat{Y} ; G) \cdot b_{1}^{(2)}(\hat{Z} ; G) \Leftrightarrow \\
& \frac{b_{1}^{(2)}(\hat{\hat{S}} ; G \times G)}{[\Gamma \times \Gamma: G \times G]} \leq \frac{b_{1}^{(2)}(\hat{Y} ; G)}{[\Gamma: G]} \cdot \frac{b_{1}^{(2)}(\hat{Z} ; G)}{[\Gamma: G]} \Leftrightarrow \\
& b_{1}^{(2)}(\hat{\hat{S}} ; \Gamma \times \Gamma) \leq b_{1}^{(2)}(\hat{Y} ; \Gamma) \cdot b_{1}^{(2)}(\hat{Z} ; \Gamma) .
\end{aligned}
$$

This finishes the proof of Theorem 23.

We will say that $S H N C$ holds for $\mathcal{S}$ if any of the inequalities in Theorem 23 is satisfied.

\section{SuRfaCEs.}

8.1. Surface systems. Generally, a surface system is a system consisting of surfaces. By a surface we mean a 2-manifold that can be with boundary and not necessarily compact, connected, or orientable. For the purposes of this paper, by a surface system $\mathcal{S}$ we will mean a particular kind of a system that arises as described below.

Let $X$ be a compact connected surface, possibly with boundary, such that $\chi(X)=-1$. Denote $\Gamma:=\pi_{1}(X)$. Since $\chi(X)<0$, it is possible to put a hyperbolic metric on $X$ with respect to which its boundary is totally convex. The universal cover $\hat{X}$ of $X$ can be identified with a convex subspace of the hyperbolic plane $\mathbb{H}^{2}$ and the $\Gamma$-action on $\hat{X}$ can be extended to an isometric $\Gamma$-action on $\mathbb{H}^{2}$ such that $\hat{X}$ is the convex hull of the limit set of $\Gamma$. Let $p_{X}: \hat{X} \rightarrow X$ be the quotient by $\Gamma$, equivalently, the universal cover map.

Given arbitrary finitely generated subgroups $A$ and $B$ of $\Gamma$, the quotients $Y^{\prime}:=A \backslash \hat{X}$ and $Z^{\prime}:=B \backslash \hat{X}$ are hyperbolic surfaces with totally geodesic boundary. The quotient maps are the covers of $X$ representing the inclusions $A \subseteq \Gamma$ and $B \subseteq \Gamma$. The maps $\alpha: Y^{\prime} \rightarrow X, \beta: Z^{\prime} \rightarrow X$, $p_{X}: \hat{X} \rightarrow X$ generate a system as in (11) but consisting of surfaces.

Let $K$ and $L$ be the convex hulls of the limit sets of $A$ and $B$ in $\mathbb{H}^{2}$, respectively, then $K \subseteq \hat{X}$ and $L \subseteq \hat{X}$. If $A$ or $B$ is trivial, then its limit set is empty; in this case we define the convex hull to be a point in $\hat{X}$. If $A$ or $B$ is infinite cyclic, the corresponding convex hull is a line. Such convex hulls still will be called surfaces, though degenerate ones.

Denote $Y:=A \backslash K \subseteq Y^{\prime}$ and $Z:=B \backslash L \subseteq Z^{\prime}$. Since $A$ and $B$ are finitely generated and $\Gamma$ has no parabolic elements, then $Y$ and $Z$ are compact surfaces with totally geodesic boundary. The above quotient maps $\alpha$ and $\beta$ can be restricted to $\alpha: Y \rightarrow X$ and $\beta: Z \rightarrow X$. The maps $\alpha: Y \rightarrow X, \beta: Z \rightarrow X$, and $p_{X}: \hat{X} \rightarrow X$ generate a system $\mathcal{S}$ as in (5) but consisting of surfaces. $\mathcal{S}$ is a subsystem of $\mathcal{S}^{\circ}$.

By a result of Scott $[21,22]$, these restricted maps $\alpha: Y \rightarrow X$ and $\beta: Z \rightarrow X$ can be extended to finite covers $\alpha: Y^{\circ} \rightarrow X$ and $\beta: Z^{\circ} \rightarrow X$. The maps $\alpha: Y^{\circ} \rightarrow X, \beta: Z^{\circ} \rightarrow X$, and $p_{X}: \hat{X} \rightarrow X$ generate a system $\mathcal{S}^{\circ}$ as in (12), but again consisting of surfaces. $\mathcal{S}$ and $\mathcal{S}^{\circ}$ will be called surface systems corresponding to $(\Gamma, A, B)$. 
One can work in the smooth category or triangulate all the surfaces involved to make them cell complexes.

8.2. Conjectures for surfaces. Swarup observed that HNC can be restated as a question about areas of surfaces. We want to ask a similar question about $\ell^{2}$-Betti numbers, which generalizes HNC.

Conjecture 3 (Surfaces). Let $\Gamma$ be the fundamental group of a connected compact surface $X$, possibly with boundary, such that $\chi(X)=-1$. Let $A$ and $B$ be finitely generated subgroups of $\pi_{1}(X)$. Then

$$
b_{1}^{(2)}(A \cap B) \leq b_{1}^{(2)}(A) \cdot b_{1}^{(2)}(B) .
$$

This conjecture implies the original HNC because one can replace graphs with homotopy equivalent compact surfaces with boundary.

Conjecture 4 (Strengthened conjecture 3). Let $\Gamma$ be the fundamental group of a compact surface $X$ with $\chi(X)=-1$, possibly with boundary, and $A$ and $B$ be its finitely generated subgroups. Then in the corresponding system $\mathcal{S}$,

$$
b_{1}^{(2)}(\hat{S} ; \Gamma) \leq b_{1}^{(2)}(\hat{Y} ; \Gamma) \cdot b_{1}^{(2)}(\hat{Z} ; \Gamma) .
$$

Similarly, this conjecture implies SHNC. We note here that if either version of the original Hanna Neumann conjecture can be proved by the methods of this paper, then the same proof should work for its stronger surface versions stated above.

The following is a complete analog of Theorem 23 for surfaces, with the same proof.

Theorem 24. Each of the following statements is equivalent to Conjecture 4.

(a) For any surface system $\mathcal{S}$ as in 8.1,

$$
b_{1}^{(2)}(\hat{S} ; \Gamma \times \Gamma) \leq b_{1}^{(2)}(\hat{Y} ; \Gamma) \cdot b_{1}^{(2)}(\hat{Z} ; \Gamma) .
$$

(a') For any surface system $\mathcal{S}$ as in 8.1,

$$
b_{1}^{(2)}(\hat{S} ; \Gamma) \leq b_{1}^{(2)}(\hat{Y} ; \Gamma) \cdot b_{1}^{(2)}(\hat{Z} ; \Gamma) .
$$

(b) If $\mathcal{S}$ is any surface system as in 8.1 and $\mathcal{S}$ is connected, then

$$
b_{1}^{(2)}(\hat{S} ; \Gamma \times \Gamma) \leq b_{1}^{(2)}(\hat{Y} ; \Gamma) \cdot b_{1}^{(2)}(\hat{Z} ; \Gamma) .
$$

(b') If $\mathcal{S}$ is any surface system as in 8.1 and $\mathcal{S}$ is connected, then

$$
b_{1}^{(2)}(\hat{S} ; \Gamma) \leq b_{1}^{(2)}(\hat{Y} ; \Gamma) \cdot b_{1}^{(2)}(\hat{Z} ; \Gamma) .
$$

(c) If $\left(\mathcal{S}^{\circ}, \mathcal{S}\right)$ is a pair of surface systems as in 8.1 and it is connected and symmetric, then

$$
b_{1}^{(2)}(\hat{S} ; \Gamma \times \Gamma) \leq b_{1}^{(2)}(\hat{Y} ; \Gamma) \cdot b_{1}^{(2)}(\hat{Z} ; \Gamma) .
$$

$\left(c^{\prime}\right)$ If $\left(\mathcal{S}^{\circ}, \mathcal{S}\right)$ is a pair of surface systems as in 8.1 and it is connected and symmetric, then

$$
b_{1}^{(2)}(\hat{S} ; \Gamma) \leq b_{1}^{(2)}(\hat{Y} ; \Gamma) \cdot b_{1}^{(2)}(\hat{Z} ; \Gamma) .
$$

(d) Any of the above statements with the additional assumption that $Y, Z, Y^{\circ}$, and $Z^{\circ}$ are orientable. 
To prove the equivalence of $(\mathrm{d})$ to the rest, start with a surface system $\mathcal{S}$ and consider orientable covers of $Y^{\circ}$ and $Z^{\circ}$. This can be done by replacing $\Gamma$ with a subgroup $\Gamma^{\prime}$ of finite index. Then replace $\mathcal{S}$ with the $\Gamma$-system induced from $\Gamma$ to $\Gamma^{\prime}$ as in 6.1 .

8.3. $\ell^{2}$-Betti numbers for surfaces. Similarly to graphs, the integrality of $\ell^{2}$-Betti numbers for surface groups can be deduced from the Atiyah conjecture for surface groups by Linnell's result. And again, we can give an elementary proof of integrality with an explicit description of the numbers.

Theorem 25. Suppose $\Gamma$ is a group.

(a) If $\Gamma$ acts freely and cocompactly on a surface $\hat{Y}$ whose components are infinite, then

$$
b_{0}^{(2)}(\hat{Y} ; \Gamma)=0 \quad \text { and } \quad b_{1}^{(2)}(\hat{Y} ; \Gamma)=-\chi(\Gamma \backslash \hat{Y}) .
$$

(b) If $\Gamma$ acts freely and cocompactly on a surface $\hat{Y}$ whose components are finite and the stabilizer of each component of $\hat{Y}$ is trivial, then

$$
b_{i}^{(2)}(\hat{Y} ; \Gamma)=b_{i}(\Gamma \backslash \hat{Y})
$$

for each $i$, where $b_{i}(\Gamma \backslash \hat{Y})$ are the usual Betti numbers of $\Gamma \backslash \hat{Y}$.

(c) If $\Gamma$ is torsion-free and it acts freely and cocompactly on a surface $\hat{Y}$, then all the $\ell^{2}$-Betti numbers $b_{i}^{(2)}(\hat{Y} ; \Gamma)$ are integers.

Proof. (a) Since each component of $\hat{Y}$ is infinite, it is easy to see that each 2-dimensional $\ell^{2}$ cycle on $\hat{Y}$ must be 0 , so the second $\ell^{2}$-Betti number is 0 . Then the proof goes word-by-word as in Lemma 13.

(b) and (c) are proved exactly as in Lemma 13.

8.4. Atomic decompositions of surfaces. Consider a surface system $\mathcal{S}$ as in 8.1 in which $Y$ and $Z$ are compact and orientable. Let $\gamma: S \rightarrow X$ be the immersion coming from $\mathcal{S}$. Then $S$ is orientable and can be decomposed as a finite union $S=\bigcup_{i} S_{i}^{\bullet}$ where each $S_{i}^{\bullet}$ is either a pair of pants or the torus with an open disc removed, and for each $i \neq j, S_{i}^{\bullet} \cap S_{j}^{\bullet}$ is a union (possibly empty) of circles that are boundary components of $S_{i}^{\bullet}$ and $S_{j}^{\bullet}$. The gluing map $\bigsqcup_{i} S_{i}^{\bullet} \rightarrow S$ can be called an atomic decomposition of $S$. Then atomic decompositions of $\hat{S}$ and of $\hat{S}$ are defined by pull-backs exactly as in 5.5.

Theorem 26. Let $\Gamma:=\pi_{1}(X)$ and $\varphi: S^{\bullet} \rightarrow S$ and $\hat{\varphi}: \hat{S}^{\bullet} \rightarrow \hat{S}$ be atomic maps of surfaces as above. Then

(a) for each $i, \beta_{1}^{(2)}\left(\mathrm{H}\left(\hat{S}_{i}^{\bullet}\right), \Gamma\right)=1$,

(b) the induced maps $\hat{\psi}_{i}: \mathrm{H}\left(\hat{S}_{i}^{\bullet}\right) \rightarrow \mathrm{H}(\hat{X})$ and $\hat{\varphi}: \mathrm{H}\left(\hat{S}^{\bullet}\right) \rightarrow \mathrm{H}(\hat{S})$ are weak isomorphisms of Hilbert $\Delta$-modules,

(c) the induced maps $\hat{\psi}_{i}: \mathrm{H}\left(\hat{\hat{S}}_{i}^{\bullet}\right) \rightarrow \mathrm{H}(\hat{X})$ and $\hat{\hat{\varphi}}: \mathrm{H}(\hat{\hat{S}} \bullet) \rightarrow \mathrm{H}(\hat{\hat{S}})$ are weak isomorphisms of Hilbert $\Gamma \times \Gamma$-modules. 
Proof. (a) follows from Theorem $25\left(\right.$ a) because $-\chi\left(S_{i}^{\bullet}\right)=1$.

(b) and (c) are proved exactly as in Theorem 16. We only need to use Theorem 11 instead of Theorem 10(1) and observe that for each $i, \varphi\left(S_{i}^{\bullet}\right) \cap \bigcup_{j \neq i} \varphi\left(S_{j}^{\bullet}\right)$ is a disjoint union of circles in $S$.

Similarly, atomic decomposition can be defined for the immersions $\alpha: Y \rightarrow X$ and $\beta: Z \rightarrow$ $X$.

\section{The diagonal approach.}

Consider a graph system $\mathcal{S}$ as in 3.6 or a surface system as in 8.1. In this section we work with $\hat{S}$, which can be viewed as the diagonal in $\hat{\hat{S}}$.

First assume that $\mathcal{S}$ is a graph system and let $\hat{Z}$ be part of $\mathcal{S}$. Let $Q \subseteq Q^{\prime}$ be $\Gamma$-invariant subgraphs of $\hat{Z}$, then

$$
\mathrm{H}(Q) \subseteq \mathrm{H}\left(Q^{\prime}\right) \subseteq \mathrm{H}(\hat{Z}) \subseteq C_{1}^{(2)}(\hat{Z})
$$

Lemma 27. If $Q \subseteq Q^{\prime}$ are $\Gamma$-invariant subgraphs of $\hat{Z}$, then

$$
\operatorname{dim}_{\Gamma}\left[\mathrm{H}(Q)^{\perp} \cap \mathrm{H}\left(Q^{\prime}\right)\right] \in \mathbb{Z} .
$$

Proof. By Lemma 13, $\operatorname{dim}_{\Gamma} \mathrm{H}(Q)$ and $\operatorname{dim}_{\Gamma} \mathrm{H}\left(Q^{\prime}\right)$ are integers, and

$$
\operatorname{dim}_{\Gamma}\left[\mathrm{H}(Q)^{\perp} \cap \mathrm{H}\left(Q^{\prime}\right)\right]=\operatorname{dim}_{\Gamma} \mathrm{H}\left(Q^{\prime}\right)-\operatorname{dim}_{\Gamma} \mathrm{H}(Q) .
$$

Lemma 28. Let $Q \subseteq Q^{\prime}$ be $\Gamma$-invariant subgraphs of $\hat{Z}$ such that $Q^{\prime} \backslash Q$ is exactly one $\Gamma$-orbit of edges. Then $\operatorname{dim}_{\Gamma}\left[\mathrm{H}(Q)^{\perp} \cap \mathrm{H}\left(Q^{\prime}\right)\right] \in[0,1]$.

Proof. Since $\mathrm{H}(Q)=\mathrm{H}\left(Q^{\prime}\right) \cap C_{1}^{(2)}(Q) \subseteq C_{1}^{(2)}(\hat{Z})$, there is a (purely algebraic) injection of $\mathcal{N}(\Gamma)$-modules

$$
\frac{\mathrm{H}\left(Q^{\prime}\right)}{\mathrm{H}(Q)}=\frac{\mathrm{H}\left(Q^{\prime}\right)}{\mathrm{H}\left(Q^{\prime}\right) \cap C_{1}^{(2)}(Q)} \hookrightarrow \frac{C_{1}^{(2)}\left(Q^{\prime}\right)}{C_{1}^{(2)}(Q)},
$$

or, if one prefers working with Hilbert modules, the orthogonal projection of $\mathrm{H}(Q)^{\perp} \cap \mathrm{H}\left(Q^{\prime}\right)$ into $C_{1}^{(2)}(Q)^{\perp} \cap C_{1}^{(2)}\left(Q^{\prime}\right)$ is injective. Then

$$
0 \leq \operatorname{dim}_{\Gamma}\left[\mathrm{H}(Q)^{\perp} \cap \mathrm{H}\left(Q^{\prime}\right)\right]=\frac{\mathrm{H}\left(Q^{\prime}\right)}{\mathrm{H}(Q)} \leq \operatorname{dim}_{\Gamma} \frac{C_{1}^{(2)}\left(Q^{\prime}\right)}{C_{1}^{(2)}(Q)}=1 .
$$

Theorem 29. Consider a graph system as in 3.6. If $Q \subseteq Q^{\prime}$ are $\Gamma$-invariant subgraphs of $\hat{Z}$ such that $Q^{\prime} \backslash Q$ is exactly one $\Gamma$-orbit of edges, then the following conditions hold.

(a) $\operatorname{dim}_{\Gamma}\left[\mathrm{H}(Q)^{\perp} \cap \mathrm{H}\left(Q^{\prime}\right)\right]$ is 0 or 1 .

(b) There exists an orthonormal $\Gamma$-basis of $\mathrm{H}(Q)^{\perp} \cap \mathrm{H}\left(Q^{\prime}\right)$ inscribed in Comp $(\hat{Z})$ (as in 4.7). 
(c) If $\mathrm{H}(Q)^{\perp} \cap \mathrm{H}\left(Q^{\prime}\right) \neq 0$ then the restriction $\hat{\beta}^{\prime}: \mathrm{H}(Q)^{\perp} \cap \mathrm{H}\left(Q^{\prime}\right) \rightarrow \mathrm{H}(\hat{X})$ of $\hat{\beta}$ is a weak isomorphism.

Proof. (a) follows from lemmas 27 and 28.

(b) If $\operatorname{dim}_{\Gamma}\left[\mathrm{H}(Q)^{\perp} \cap \mathrm{H}\left(Q^{\prime}\right)\right]=0$, the space is 0 and has the obvious empty basis, so we can assume $\operatorname{dim}_{\Gamma}\left[\mathrm{H}(Q)^{\perp} \cap \mathrm{H}\left(Q^{\prime}\right)\right]=1$. It suffices to prove the statement in the case when the $\Gamma$-action on $\operatorname{Comp}(\hat{Z})$ is transitive. Take $L \in \operatorname{Comp}(\hat{Z})$ and denote $B:=\operatorname{Stab}_{\Gamma}(L)$.

By Theorem 7(e) we have the orthogonal decompositions

$$
\begin{aligned}
& \mathrm{H}(\hat{Z})=\bigoplus_{g \in \Gamma / B} \mathrm{H}(g L), \\
& \mathrm{H}(Q)=\bigoplus_{g \in \Gamma / B} \mathrm{H}(Q) \cap \mathrm{H}(g L)=\bigoplus_{g \in \Gamma / B} \mathrm{H}(Q \cap g L), \\
& \mathrm{H}\left(Q^{\prime}\right)=\bigoplus_{g \in \Gamma / B} \mathrm{H}\left(Q^{\prime}\right) \cap \mathrm{H}(g L)=\bigoplus_{g \in \Gamma / B} \mathrm{H}\left(Q^{\prime} \cap g L\right) .
\end{aligned}
$$

This implies

$$
\begin{aligned}
& \mathrm{H}(Q)^{\perp} \cap \mathrm{H}\left(Q^{\prime}\right)=\bigoplus_{g \in \Gamma / B}[\mathrm{H}(Q \cap g L)]^{\perp} \cap\left[\mathrm{H}\left(Q^{\prime} \cap g L\right)\right] \\
& =\bigoplus_{g \in \Gamma / B} g\left([\mathrm{H}(Q \cap L)]^{\perp} \cap\left[\mathrm{H}\left(Q^{\prime} \cap L\right)\right]\right) .
\end{aligned}
$$

Denote

$$
W:=[\mathrm{H}(Q \cap L)]^{\perp} \cap\left[\mathrm{H}\left(Q^{\prime} \cap L\right)\right],
$$

then $W$ is $B$-invariant and

$$
\begin{aligned}
& \mathrm{H}(Q)^{\perp} \cap \mathrm{H}\left(Q^{\prime}\right)=\bigoplus_{g \in \Gamma / B} g W=\operatorname{Ind}_{B}^{\Gamma} W \\
& \operatorname{dim}_{B} W=\operatorname{dim}_{\Gamma} \operatorname{Ind}_{B}^{\Gamma} W=\operatorname{dim}_{\Gamma} \mathrm{H}(Q)^{\perp} \cap \mathrm{H}\left(Q^{\prime}\right)=1 .
\end{aligned}
$$

Since $B \leq \Gamma$, then $B$ is free. If $B$ is trivial or cyclic, then $\mathrm{H}\left(Q^{\prime}\right) \subseteq \mathrm{H}(\hat{Z})=0$ and we can take the empty basis for $\mathrm{H}(Q)^{\perp} \cap \mathrm{H}\left(Q^{\prime}\right)$. Now assume that $B$ is of rank $\geq 2$, then it is an ICC group, that is every nontrivial conjugacy class in $B$ is infinite. Then $\mathcal{N}(B)$ is a $I I_{1}$ factor ([12, Proposition 1.4.1]). Hilbert modules over $I I_{1}$ factors are uniquely determined by their dimension, therefore $W$ is isometrically isomorphic to $\ell^{2}(B)$ as a Hilbert $B$-module. This allows choosing an orthonormal $B$-basis in $W \subseteq \mathrm{H}(L)$ (consisting of one vector). This basis is inscribed in $\operatorname{Comp}(\hat{Z})$. The same vector forms an orthonormal $\Gamma$-basis of $\mathrm{H}(Q)^{\perp} \cap \mathrm{H}\left(Q^{\prime}\right)=\operatorname{Ind}_{B}^{\Gamma} W$.

(c) Since

$$
\operatorname{dim}_{\Gamma} \mathrm{H}(Q)^{\perp} \cap \mathrm{H}\left(Q^{\prime}\right)=1=\operatorname{dim}_{\Gamma} \mathrm{H}(\hat{X}),
$$

it suffices to show that $\hat{\beta}^{\prime}: \mathrm{H}(Q)^{\perp} \cap \mathrm{H}\left(Q^{\prime}\right) \rightarrow \mathrm{H}(\hat{X})$ is injective.

Let $f_{Q^{\prime}}:=\left[\partial_{1} \mid q_{Q^{\prime}}\right]$ be the stack of the maps

$$
\partial_{1}: C_{1}^{(2)}(\hat{Z}) \rightarrow C_{0}^{(2)}(\hat{Z}) \quad \text { and } \quad q_{Q^{\prime}}: C_{1}^{(2)}(\hat{Z}) \rightarrow C_{1}^{(2)}(\hat{Z}),
$$


where $\partial_{1}$ is the boundary map and $q_{Q^{\prime}}$ is defined on the basis $\Sigma_{1}(\hat{Z})$ by

$$
q_{Q^{\prime}}(e):= \begin{cases}e & \text { if } e \notin \Sigma_{1}\left(Q^{\prime}\right), \\ 0 & \text { if } e \in \Sigma_{1}\left(Q^{\prime}\right) .\end{cases}
$$

The map $f_{Q}:=\left[\partial_{1} \mid q_{Q}\right]$ is defined similarly to $f_{Q^{\prime}}$. We have

$$
\begin{aligned}
& \operatorname{Ker} f_{Q^{\prime}}=\operatorname{Ker} \partial_{1} \cap \operatorname{Ker} q_{Q^{\prime}}=\mathrm{H}(\hat{Z}) \cap C_{1}^{(2)}\left(Q^{\prime}\right)=\mathrm{H}\left(Q^{\prime}\right), \\
& \left(\operatorname{Ker} f_{Q}\right)^{\perp}=\mathrm{H}(Q)^{\perp},
\end{aligned}
$$

where the orthogonal complements are taken in $C_{1}^{(2)}(\hat{Z})$.

The maps $\partial_{1}, q_{Q}, q_{Q^{\prime}}$ and $\hat{\beta}$ are $\Gamma$-equivariant and they send basis elements of their domains to finite linear combinations of basis elements in their target, hence $f_{Q}$ and $\left[f_{Q^{\prime}} \mid \hat{\beta}\right]$ are such as well.

$\operatorname{Ker} \hat{\beta}^{\prime}=\mathrm{H}(Q)^{\perp} \cap \mathrm{H}\left(Q^{\prime}\right) \cap \operatorname{Ker} \hat{\beta}=\left(\operatorname{Ker} f_{Q}\right)^{\perp} \cap \operatorname{Ker} f_{Q^{\prime}} \cap \operatorname{Ker} \hat{\beta}=\left(\operatorname{Ker} f_{Q}\right)^{\perp} \cap \operatorname{Ker}\left[f_{Q^{\prime}} \mid \hat{\beta}\right]$, then by Theorem 10(2),

$$
\operatorname{dim}_{\Gamma} \operatorname{Ker} \hat{\beta}^{\prime}=\operatorname{dim}_{\Gamma}\left[\left(\operatorname{Ker} f_{Q}\right)^{\perp} \cap \operatorname{Ker}\left[f_{Q^{\prime}} \mid \hat{\beta}\right]\right] \in \mathbb{Z} .
$$

Then $\operatorname{dim}_{\Gamma} \operatorname{Ker} \hat{\beta}^{\prime}$ is 0 or 1 . The basis in part (b) above is inscribed into $\operatorname{Comp}(\hat{Z})$, and the restriction of $\hat{\beta}$ to each component of $\hat{Z}$ is an isomorphsim. This implies that $\hat{\beta}^{\prime}$ maps each basis element to a non-zero element in $\mathrm{H}(\hat{X})$, so $\hat{\beta}^{\prime}$ is not the zero map. Then by the additivity of dimension,

$$
\operatorname{dim}_{\Gamma} \operatorname{Ker} \hat{\beta}^{\prime}<\operatorname{dim}_{\Gamma}\left[\mathrm{H}(Q)^{\perp} \cap \mathrm{H}\left(Q^{\prime}\right)\right]=1,
$$

hence $\operatorname{dim}_{\Gamma} \operatorname{Ker} \hat{\beta}^{\prime}=0$ and $\hat{\beta}^{\prime}$ is injective.

Theorem 30. For any graph system $\mathcal{S}$ as in 3.6 there exist orthonormal $\Gamma$-bases $\mathcal{B}$ of $\mathrm{H}(\hat{Z})$ and $\mathcal{B}^{\circ}$ of $\mathrm{H}\left(\hat{Z}^{\circ}\right)$ such that

(1) $\mathcal{B} \subseteq \mathcal{B}^{\circ}$,

(2) $\mathcal{B}$ is inscribed in $\operatorname{Comp}(\hat{Z})$,

(3) $\mathcal{B}^{\circ}$ is inscribed in $\operatorname{Comp}\left(\hat{Z}^{\circ}\right)$, and

(4) for each $b \in \mathcal{B}^{\circ}$, the restricted map $\hat{\beta}: \overline{\operatorname{span}}(\Gamma b) \rightarrow \mathrm{H}(\hat{X})$ is a weak isomorphism.

Proof. Choose a finite sequence of $\Gamma$-invariant subgraphs

$$
Q_{0} \subseteq \ldots \subseteq Q_{l} \subseteq \ldots \subseteq Q_{n}=\hat{Z}^{\circ}
$$

such that $Q_{0}$ has no edges, $Q_{l}=\hat{Z}$ for some $l$, and each $Q_{i}$ is obtained from $Q_{i+1}$ by removing one $\Gamma$-orbit of edges.

We have

$$
0=\mathrm{H}\left(Q_{0}\right) \subseteq \ldots \subseteq \mathrm{H}\left(Q_{l}\right) \subseteq \ldots \mathrm{H}\left(Q_{n}\right)=\mathrm{H}\left(\hat{Z}^{\circ}\right) .
$$

Inductively on $i$, for each $i$ such that $\mathrm{H}\left(Q_{i}\right) \neq \mathrm{H}\left(Q_{i+1}\right)$, add a $\Gamma$-basis vector constructed in Theorem 29(b). By Theorem 29(c), $\hat{\beta}: \overline{\operatorname{span}}(\Gamma b) \rightarrow \mathrm{H}(\hat{X})$ is a weak isomorphism for each $b \in \mathcal{B}^{\circ}$. 
It should be possible to generalize the argument of Theorem 29 to surfaces by adding one atom of a surface at a time instead of edges in a graph, but the proof becomes more involved. The reason is that if $Q$ is a union of atoms of a surface $\hat{Z}$ and $Q^{\prime}$ is obtained from $Q$ by gluing another atom, then $\mathrm{H}(Q)$ is not automatically a subset of $\mathrm{H}\left(Q^{\prime}\right)$. So in order to state an analog of Thorem 29 for surfaces, one would need first to replace $\mathrm{H}(Q)$ and $\mathrm{H}\left(Q^{\prime}\right)$ with their images under the injective maps $\mathrm{H}(Q) \rightarrow \mathrm{H}(\hat{Z})$ and $\mathrm{H}\left(Q^{\prime}\right) \rightarrow \mathrm{H}(\hat{Z})$. This would imply an analog of Theorem 30 for surfaces. To avoid technicalities we content ourselves with proving the following weaker version of Theorem 30 .

Theorem 31. For each surface system $\mathcal{S}$ as in 8.1, there exist (not necessarily orthonormal) $\Gamma$-bases $\mathcal{B}$ of $\mathrm{H}(\hat{Z})$ and $\mathcal{B}^{\circ}$ of $\mathrm{H}\left(\hat{Z}^{\circ}\right)$ such that

(1) $\mathcal{B} \subseteq \mathcal{B}^{\circ}$

(2) $\mathcal{B}$ is inscribed in $\operatorname{Comp}(\hat{Z})$,

(3) $\mathcal{B}^{\circ}$ is inscribed in $\operatorname{Comp}\left(\hat{Z}^{\circ}\right)$, and

(4) for each $b \in \mathcal{B}^{\circ}$, the restricted map $\hat{\beta}: \overline{\operatorname{span}}(\Gamma b) \rightarrow \mathrm{H}(\hat{X})$ is a densely defined injective map whose image is dense in $\mathrm{H}(\hat{X})$.

Proof. Take an atomic decomposition $\hat{Z}=\bigcup \hat{Z}_{i}^{\bullet}$ of $\hat{Z}$ as in 8.4. For each atom $\hat{Z}_{i}^{\bullet}$ pick an orthonormal $\Gamma$-basis inscribed in $\operatorname{Comp}\left(\hat{Z}_{i}^{\bullet}\right)$. The disjoint union of such gives an orthonormal $\Gamma$-basis of $\operatorname{Comp}\left(\hat{Z}^{\bullet}\right)$. Its image under the weak isomorphism $\hat{\varphi}: \mathrm{H}\left(\hat{Z}^{\bullet}\right) \rightarrow \mathrm{H}(\hat{Z})$ is a $\Gamma$-basis of $\mathrm{H}(\hat{Z})$ inscribed in $\operatorname{Comp}(\hat{Z})$. This extends similarly to a basis of $\mathrm{H}\left(\hat{Z}^{\circ}\right)$. The last property (4) follows from the fact that $\hat{\psi}_{i}: \mathrm{H}\left(\hat{Z}_{i}^{\bullet}\right) \rightarrow \mathrm{H}(\hat{X})$ is a weak isomorphism (Theorem 26(b)) and the commutativity of diagram (20).

The next result says that a stronger version of Theorem 30 would imply SHNC. It uses changes of Hilbert structures discussed in 4.5.

Theorem 32 (compatible Hilbert structures). Suppose

- $\mathcal{S}$ is a graph system as in 3.6 or a surface system as in 8.1,

- $\mathrm{H}(\hat{X})$ is given a Hilbert $\Gamma$-module structure, denoted $\mathrm{H}^{\prime}(\hat{X})$,

- $\mathrm{H}(\hat{Z})$ is given the Hilbert structure $\mathrm{H}^{\prime}(\hat{Z})$ lifted from $\mathrm{H}^{\prime}(\hat{X})$ by pulling back the inner product by the map $\mathrm{H}(L) \hookrightarrow \mathrm{H}^{\prime}(\hat{X})$ for each $L \in \operatorname{Comp}(\hat{Z})$ and declaring $\mathrm{H}(L)$ and $\mathrm{H}\left(L^{\prime}\right)$ orthogonal for distinct $L, L^{\prime} \in \operatorname{Comp}(\hat{Z})$,

- $\mathrm{H}^{\prime}(\hat{Z})$ admits an orthonormal $\Gamma$-basis $\mathcal{B}$ inscribed in $\operatorname{Comp}\left(\hat{Z}^{\circ}\right)$ such that for each $b \in \mathcal{B}$ the restricted map $\hat{\beta}: \overline{\operatorname{span}}(\Gamma b) \rightarrow \mathrm{H}(\hat{X})$ is an isometric isomorphism.

Then SHNC holds for $\mathcal{S}$, i.e. $b_{1}^{(2)}(\hat{S} ; \Gamma) \leq b_{1}^{(2)}(\hat{Y} ; \Gamma) \cdot b_{1}^{(2)}(\hat{Z} ; \Gamma)$.

Proof. Denote $l:=\operatorname{dim}_{\Gamma} \mathrm{H}(\hat{Z})=\operatorname{dim}_{\Gamma} \mathrm{H}^{\prime}(\hat{Z})$, then $\mathcal{B}=\left\{b_{i} \mid i=1, \ldots, l\right\}$. The map $\hat{\nu}: \hat{S}^{\circ} \rightarrow \hat{Z}^{\circ}$ is a $\Gamma$-leafage in the sense of 2.4. Let $k^{\circ}$ be the multiplicity of the cover $\alpha: Y^{\circ} \rightarrow X$, then the cover leafage $\hat{\nu}: \hat{S}^{\circ} \rightarrow \hat{Z}^{\circ}$ has multiplicity $k^{\circ}$. By the inclusion $\mathrm{H}^{\prime}(\hat{Z}) \subseteq \mathrm{H}^{\prime}\left(\hat{Z}^{\circ}\right)$ we view $\mathcal{B}$ as a subset of $\mathrm{H}^{\prime}\left(\hat{Z}^{\circ}\right)$.

We lift the Hilbert structure from $\mathrm{H}^{\prime}(\hat{X})$ to $\mathrm{H}\left(\hat{Y}^{\circ}\right)$ and to $\mathrm{H}\left(\hat{S}^{\circ}\right)$, and denote these structures $\mathrm{H}^{\prime}\left(\hat{Y}^{\circ}\right)$ and $\mathrm{H}^{\prime}\left(\hat{S}^{\circ}\right)$, respectively. 
Let $\hat{\nu}^{\uparrow}(\mathcal{B})$ be the lift of $\mathcal{B}$ to $\hat{S}^{\circ}$ via $\hat{\nu}: \hat{S}^{\circ} \rightarrow \hat{Z}^{\circ}$ as in 4.7 . Each $b_{i} \in \mathcal{B}$ lifts to $k^{\circ}$ functions

$$
\hat{\nu}^{\uparrow}\left(b_{i}\right)=\left\{b_{i j} \mid j=1, \ldots, k^{\circ}\right\} \subseteq \mathrm{H}^{\prime}\left(\hat{Y}^{\circ} \hat{\square} \hat{Z}\right) \subseteq \mathrm{H}^{\prime}\left(\hat{S}^{\circ}\right) .
$$

We now need the following lemma.

Lemma 33. The full lift

$$
\hat{\nu}^{\uparrow}(\mathcal{B})=\left\{b_{i j} \mid i=1, \ldots, l, j=1, \ldots, k^{\circ}\right\}
$$

is an orthonormal $\Gamma$-basis of $\mathrm{H}^{\prime}\left(\hat{Y}^{\circ} \hat{\square} \hat{Z}\right) \subseteq \mathrm{H}^{\prime}\left(\hat{S}^{\circ}\right)$.

Proof. Take any $K^{\circ} \in \operatorname{Comp}\left(\hat{Y}^{\circ}\right)$, then $K^{\circ} \cong \hat{X}, K^{\circ} \hat{\square} \hat{Z} \cong \hat{X} \hat{\square} \hat{Z} \cong \hat{Z}$, and $\mathrm{H}^{\prime}\left(K^{\circ} \hat{\square} \hat{Z}\right) \cong$ $\mathrm{H}^{\prime}(\hat{Z})$. The horizontal arrows in the diagram

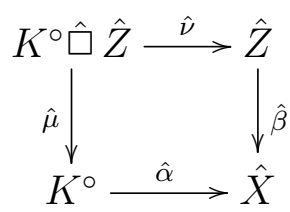

are isomorphisms. $\hat{\nu}^{\uparrow}(\Gamma \mathcal{B}) \cap \mathrm{H}^{\prime}\left(K^{\circ} \hat{\square} \hat{Z}\right)$ is the lift of $\Gamma \mathcal{B}$ under the isometric isomorphism $\hat{\nu}: \mathrm{H}^{\prime}\left(K^{\circ} \hat{\square} \hat{Z}\right) \rightarrow \mathrm{H}^{\prime}(\hat{Z})$. Since $\Gamma \mathcal{B}$ is an orthonormal $\Gamma$-invariant basis of $\mathrm{H}^{\prime}(\hat{Z})$, then $\hat{\nu}^{\uparrow}(\Gamma \mathcal{B}) \cap$ $\mathrm{H}^{\prime}\left(K^{\circ} \hat{\square} \hat{Z}\right)$ is an orthonormal basis of $\mathrm{H}^{\prime}\left(K^{\circ} \hat{\square} \hat{Z}\right)$. Combining these over all $K^{\circ} \in \operatorname{Comp}\left(\hat{Y}^{\circ}\right)$ shows that $\hat{\nu}^{\uparrow}(\Gamma \mathcal{B})$ is an orthonormal $\Gamma$-invariant basis of

$$
\mathrm{H}^{\prime}\left(\hat{Y}^{\circ} \hat{\square} \hat{Z}\right)=\bigoplus_{K^{\circ} \in \operatorname{Comp}\left(\hat{Y}^{\circ}\right)} \mathrm{H}^{\prime}\left(K^{\circ} \hat{\square} \hat{Z}\right) .
$$

Since $\Gamma$ acts on $\Gamma \mathcal{B}$ freely, then $\Gamma$ acts on $\hat{\nu}^{\uparrow}(\Gamma \mathcal{B})$ freely. Obviously, $\hat{\nu}^{\uparrow}(\mathcal{B}) \subseteq \hat{\nu}^{\uparrow}(\Gamma \mathcal{B})$. Each $\Gamma$-orbit in $\Gamma \mathcal{B}$ intersects $\mathcal{B}$ exactly once. This implies that each $\Gamma$-orbit in $\Gamma \mathcal{B}$ intersects $\hat{\nu}^{\uparrow}(\mathcal{B})$ exactly once. This implies the lemma.

Note that $\mathrm{H}^{\prime}(\hat{S}) \subseteq \mathrm{H}^{\prime}\left(\hat{Y}^{\circ} \hat{\square} \hat{Z}\right)$. By (13) and the above lemma we have

$$
\left.b_{1}^{(2)}(\hat{S} ; \Gamma)=\operatorname{dim}_{\Gamma} \mathrm{H}^{\prime}(\hat{S})=\sum_{i j}\left\langle b_{i j}, \operatorname{pr}\left[b_{i j}, \mathrm{H}^{\prime}(\hat{S})\right]\right)\right\rangle,
$$

where $p r$ is the orthogonal projection. Let $M_{i j}^{\circ}$ be the component of $\hat{S}^{\circ}$ containing $\operatorname{supp}\left(b_{i j}\right)$, then $b_{i j} \in \mathrm{H}^{\prime}\left(M_{i j}^{\circ}\right)$. Denote $M_{i j}:=M_{i j}^{\circ} \cap \hat{S}$. Since $\mathrm{H}^{\prime}\left(\hat{S}^{\circ}\right)$ is the orthogonal sum of $\mathrm{H}^{\prime}\left(M^{\circ}\right)$ over $M^{\circ} \in \operatorname{Comp}\left(\hat{S}^{\circ}\right)$ and $\mathrm{H}^{\prime}(\hat{S})$ is the orthogonal sum of $\mathrm{H}^{\prime}\left(M^{\circ} \cap \hat{S}\right)$ over $M^{\circ} \in \operatorname{Comp}\left(\hat{S}^{\circ}\right)$, then $b_{i j}$ is orthogonal to $\mathrm{H}^{\prime}\left(M^{\circ} \cap \hat{S}\right)$ when $M^{\circ} \neq M_{i j}^{\circ}$. Then we have

$$
\left.\sum_{i j}\left\langle b_{i j}, \operatorname{pr}\left[b_{i j}, \mathrm{H}^{\prime}(\hat{S})\right]\right)\right\rangle=\sum_{i j}\left\langle b_{i j}, \operatorname{pr}\left[b_{i j}, \mathrm{H}^{\prime}\left(M_{i j}\right)\right]\right\rangle .
$$

Since $b_{i j} \in \mathrm{H}^{\prime}\left(M_{i j}^{\circ}\right), \mathrm{H}^{\prime}\left(M_{i j}\right) \subseteq \mathrm{H}^{\prime}\left(M_{i j}^{\circ}\right)$, and $\hat{\mu}$ maps $\mathrm{H}^{\prime}\left(M_{i j}^{\circ}\right)$ isometrically onto $\mathrm{H}^{\prime}\left(K^{\circ}\right)$ for some $K^{\circ} \in \operatorname{Comp}\left(\hat{Y}^{\circ}\right)$, we have 


$$
\sum_{i j}\left\langle b_{i j}, \operatorname{pr}\left[b_{i j}, \mathrm{H}^{\prime}\left(M_{i j}\right)\right]\right\rangle=\sum_{i j}\left\langle\hat{\mu}\left(b_{i j}\right), \operatorname{pr}\left[\hat{\mu}\left(b_{i j}\right), \mathrm{H}^{\prime}\left(\hat{\mu}\left(M_{i j}\right)\right)\right]\right\rangle .
$$

Since $\hat{\mu}\left(M_{i j}\right) \subseteq \hat{Y}$

$$
\sum_{i j}\left\langle\hat{\mu}\left(b_{i j}\right), \operatorname{pr}\left[\hat{\mu}\left(b_{i j}\right), \mathrm{H}^{\prime}\left(\hat{\mu}\left(M_{i j}\right)\right)\right]\right\rangle \leq \sum_{i j}\left\langle\hat{\mu}\left(b_{i j}\right), \operatorname{pr}\left[\hat{\mu}\left(b_{i j}\right), \mathrm{H}^{\prime}(\hat{Y})\right]\right\rangle .
$$

By Lemma 33, $\hat{\nu}^{\uparrow}\left(b_{i}\right) \subseteq \hat{\nu}^{\uparrow}(\mathcal{B})$ in (31) is an orthonormal $\Gamma$-subbasis in $H^{\prime}\left(\hat{Y}^{\circ} \hat{\square} \hat{Z}\right)$ for each $i$.

Lemma 34. Under the assumptions of Theorem 32, for each $i, \hat{\mu}$ maps the orthonormal $\Gamma$ subbasis $\hat{\nu}^{\uparrow}\left(b_{i}\right)$ bijectively onto an orthonormal $\Gamma$-basis of $\mathrm{H}^{\prime}\left(\hat{Y}^{\circ}\right)$.

Proof. By the assumption of Theorem 32, for each $i, \hat{\beta}$ maps the orthonormal set (subbasis) $\Gamma b_{i}$ bijectively onto an orthonormal basis of $\mathrm{H}^{\prime}(\hat{X})$. Then for each $K^{\circ} \in \operatorname{Comp}\left(\hat{Y}^{\circ}\right)$, by the isomorphisms in diagram (32), $\hat{\mu}$ maps $\hat{\nu}^{\uparrow}\left(\Gamma b_{i}\right) \cap H^{\prime}\left(K^{\circ} \hat{\square} \hat{Z}\right)$ bijectively onto an orthonormal basis of $\mathrm{H}^{\prime}\left(K^{\circ}\right)$. Combining these sets over all $K^{\circ} \in \operatorname{Comp}\left(\hat{Y}^{\circ}\right)$ we see that $\hat{\mu} \operatorname{maps} \Gamma \hat{\nu}^{\uparrow}\left(b_{i}\right)=\hat{\nu}^{\uparrow}\left(\Gamma b_{i}\right)$ bijectively onto an orthonormal basis of $\mathrm{H}^{\prime}\left(\hat{Y}^{\circ}\right)$. This implies the statement of the lemma.

This lemma implies

$$
\begin{aligned}
& \sum_{i j}\left\langle\hat{\mu}\left(b_{i j}\right), \operatorname{pr}\left[\hat{\mu}\left(b_{i j}\right), \mathrm{H}^{\prime}(\hat{Y})\right]\right\rangle=\sum_{i} \operatorname{dim}_{\Gamma} \mathrm{H}^{\prime}(\hat{Y}) \\
& =\operatorname{dim}_{\Gamma} \mathrm{H}^{\prime}(\hat{Y}) \cdot l=\operatorname{dim}_{\Gamma} \mathrm{H}^{\prime}(\hat{Y}) \cdot \operatorname{dim}_{\Gamma} \mathrm{H}^{\prime}(\hat{Z}) \\
& =b_{1}^{(2)}(\hat{Y} ; \Gamma) \cdot b_{1}^{(2)}(\hat{Z} ; \Gamma) .
\end{aligned}
$$

Combining the above equalities and inequalities gives

$$
b_{1}^{(2)}(\hat{S} ; \Gamma) \leq b_{1}^{(2)}(\hat{Y} ; \Gamma) \cdot b_{1}^{(2)}(\hat{Z} ; \Gamma),
$$

which is equivalent to SHNC by Theorem 23. This finishes the proof of Theorem 32.

Considering the standard Hilbert structure on $\mathrm{H}(\hat{X})$ in Theorem 32 is not enough, as the following example shows.

Example. Consider a graph system $\mathcal{S}$ in which $\beta: Z \rightarrow X$ is the following immersion of graphs.

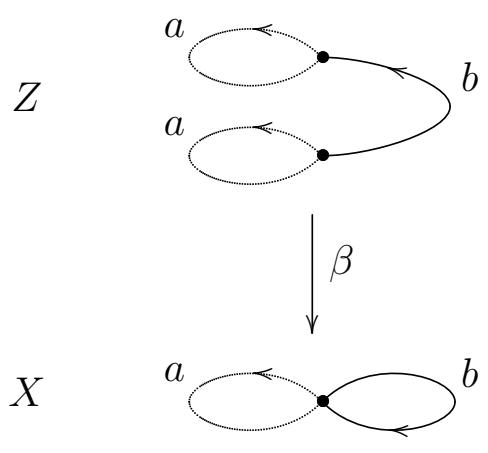


Consider the corresponding $\hat{Z}$ and $\hat{Z}$. Both $\mathrm{H}(\hat{Z})$ and $\mathrm{H}(\hat{X})$ are isomorphic to $\ell^{2}(\Gamma)$. It is easy to check that the map $\hat{\beta}: \mathrm{H}(\hat{Z}) \rightarrow \mathrm{H}(\hat{X})$ is a weak isomorphism, but not an isometric isomorphism. Equivalently, there is no orthonormal $\Gamma$-basis of $\mathrm{H}(\hat{Z})$ inscribed in $\operatorname{Comp}(\hat{Z})$ that is mapped to an orthonormal $\Gamma$-basis of $\mathrm{H}(\hat{X})$ under $\hat{\beta}$. So the standard Hilbert structures on $\mathrm{H}(\hat{X})$ and $\mathrm{H}(\hat{Z})$ do not satisfy the assumptions of Theorem 32 . Since $\operatorname{dim}_{\Gamma} \mathrm{H}(\hat{Z})=1$, this can be easily fixed by replacing $\mathrm{H}(\hat{X})$ with the Hilbert structure $\mathrm{H}^{\prime}(\hat{X})$ induced from $\mathrm{H}(\hat{Z})$ by the weak isomorphism $\hat{\beta}: \mathrm{H}(\hat{Z}) \rightarrow \mathrm{H}(\hat{X})$. Then Theorem 32 applies.

Corollary 35. If $\mathcal{S}$ is a graph system or a surface system such that $b_{1}^{(2)}(\hat{Z} ; \Gamma)=1$ then $b_{1}^{(2)}(\hat{S} ; \Gamma) \leq b_{1}^{(2)}(\hat{Y} ; \Gamma) \cdot b_{1}^{(2)}(\hat{Z} ; \Gamma)=b_{1}^{(2)}(\hat{Y} ; \Gamma)$.

Proof. The argument in the above example works for any graph or surface $Z$ such that $b_{1}^{(2)}(\hat{Z} ; \Gamma)=$ 1: modify the Hilbert structure on $\mathrm{H}(\hat{X})$, then apply Theorem 32.

This gives an alternative proof of the result of Tardos in [25]: if $B$ is of rank 2 then SHNC holds for $(\Gamma, A, B)$; and it generalizes that result to surfaces. A similar result can be proved for $\Gamma$-complexes, where $\Gamma$ is any group satisfying the Atiyah conjecture.

\section{THE SQUARE APPROACH.}

In this section we exhibit an approach to the conjecture that makes use of $\hat{S}, \hat{S}^{\circ}$, and $\Gamma \times \Gamma$.

10.1. Diagonals in $\Gamma \times \Gamma$. Let $\Gamma$ be a group and $\Delta:=\{(g, g) \mid g \in \Gamma\} \subseteq \Gamma \times \Gamma$. By a diagonal in $\Gamma \times \Gamma$ we will mean a left coset of $\Delta$ in the group $\Gamma \times \Gamma$.

We will always identify $\ell^{2}(\Gamma \times \Gamma)$ with $\ell^{2}(\Gamma) \hat{\otimes} \ell^{2}(\Gamma)$. For each diagonal $D \in(\Gamma \times \Gamma) / \Delta$, $\ell^{2}(D)$ is naturally a subspace of $\ell^{2}(\Gamma \times \Gamma)$. $\Gamma \times \Gamma$ acts on the set of diagonals $(\Gamma \times \Gamma) / \Delta$ by left multiplication.

We will say that a family $\mathcal{C} \subseteq \ell^{2}(\Gamma \times \Gamma)$ is inscribed in $(\Gamma \times \Gamma) / \Delta$ if for each $c \in \mathcal{C}$ there exists $D \in(\Gamma \times \Gamma) / \Delta$ such that $\operatorname{supp}(c) \subseteq D$.

Let $G$ be a normal subgroup of $\Gamma$ of finite index and denote $n:=[\Gamma, G]$.

Lemma 36. The set of $G \times G$ orbits in $(\Gamma \times \Gamma) / \Delta$ is the same as the set of $G \times 1$-orbits in $(\Gamma \times \Gamma) / \Delta$.

Proof. The statement of the lemma is equivalent to saying that for each $(a, b) \in \Gamma \times \Gamma$, the $G \times G$-orbit of the diagonal $(a, b) \Delta$ coincides with the $G \times 1$-orbit of the same diagonal, i.e. that

$$
(G \times G) \cdot(a, b) \Delta=(G \times 1) \cdot(a, b) \Delta .
$$


Each element of the left hand side is of the form $(g, h)(a, b) \Delta$ for some $g, h \in G$. Denote $f:=g a b^{-1} h b a^{-1}$. Since $g, h \in G \triangleleft \Gamma$, we have $f \in G$.

$$
\begin{aligned}
& f=g a b^{-1} h b a^{-1} \quad \Leftrightarrow \quad a^{-1} g^{-1} f a=b^{-1} h b \\
& \Leftrightarrow \quad\left(a^{-1} g^{-1} f a, b^{-1} h b\right)(1,1) \in \Delta \\
& \Leftrightarrow \quad\left(a^{-1} g^{-1} f a, b^{-1} h b\right) \Delta=\Delta \\
& \Leftrightarrow \quad(g a, h b) \Delta=(f a, b) \Delta \\
& \Leftrightarrow \quad(g, h)(a, b) \Delta=(f, 1)(a, b) \Delta,
\end{aligned}
$$

hence $(g, h)(a, b) \Delta \in(G \times 1) \cdot(a, b) \Delta$. The opposite inclusion is obvious.

Let $Q \subseteq \Gamma$ be a set of right coset representatives for $G \backslash \Gamma$.

Lemma 37. (a) The following maps are bijections.

$$
\begin{aligned}
\Gamma \rightarrow(\Gamma \times \Gamma) / \Delta, & g & \mapsto(g, 1) \Delta, \\
\Gamma \rightarrow(\Gamma \times \Gamma) / \Delta, & g & \mapsto(1, g) \Delta .
\end{aligned}
$$

Equivalently, the left $\Gamma \times 1$ - and $1 \times \Gamma$-actions on $(\Gamma \times \Gamma) / \Delta$ are free and transitive.

(b) The following composition is a bijection.

$$
\begin{aligned}
& G \times Q \rightarrow \Gamma \rightarrow(\Gamma \times \Gamma) / \Delta, \\
& (g, q) \mapsto g q \mapsto(g q, 1) \Delta .
\end{aligned}
$$

Proof. (a) Injectivity follows from the equivalences

$$
\begin{aligned}
& (g, 1) \Delta=(h, 1) \Delta \quad \Leftrightarrow \quad\left(g^{-1} h, 1\right) \Delta=\Delta \\
& \Leftrightarrow\left(g^{-1} h, 1\right)(1,1) \in \Delta \quad \Leftrightarrow \quad g^{-1} h=1 \quad \Leftrightarrow \quad g=h .
\end{aligned}
$$

Surjectivity follows from Lemma 36 for $G:=\Gamma$ :

$$
(\Gamma \times 1) \cdot \Delta=(\Gamma \times \Gamma) \cdot \Delta=(\Gamma \times \Gamma) / \Delta .
$$

The bijectivity of the second map is similar.

(b) follows from (a) because the first map $G \times Q \rightarrow \Gamma$ is bijective.

Denote

$$
\operatorname{Diag}_{G}^{\Gamma}:=\{(q, 1) \Delta \mid q \in Q\} \subseteq(\Gamma \times \Gamma) / \Delta
$$

\section{Lemma 38.}

(a) $\# \operatorname{Diag}_{G}^{\Gamma}=n$.

(b) $\operatorname{Diag}_{G}^{\Gamma}$ intersects each $G \times G$-orbit in $(\Gamma \times \Gamma) / \Delta$ exactly once, i.e. $\operatorname{Diag}_{G}^{\Gamma}$ is a set of representatives of the $G \times G$-orbits in $(\Gamma \times \Gamma) / \Delta$.

Proof. Lemma 37(b) implies that $\# \operatorname{Diag}_{G}^{\Gamma}=\# Q=n$ and $\operatorname{Diag}_{G}^{\Gamma}$ intersects each $G \times 1$-orbit in $(\Gamma \times \Gamma) / \Delta$ exactly once. By Lemma 36, the $G \times 1$-orbits coincide with the $G \times G$-orbits. 
10.2. Ordered $G \times G$-bases in $\ell^{2}(\Gamma \times \Gamma)$.

Lemma 39. For any $G \times G$-basis $\mathcal{C}$ of $\ell^{2}(\Gamma \times \Gamma)$ inscribed in $(\Gamma \times \Gamma) / \Delta$ there exists a $G \times G$-basis $\mathcal{C}^{\prime}$ such that

- $\mathcal{C}^{\prime} \subseteq(G \times G) \mathcal{C}$ and

- $\mathcal{C}^{\prime}$ is inscribed in $\operatorname{Diag}_{G}^{\Gamma}$.

Proof. Since $\mathcal{C}$ is inscribed in $(\Gamma \times \Gamma) / \Delta$ then $(G \times G) \mathcal{C}$ is also inscribed in $(\Gamma \times \Gamma) / \Delta$. The left $G \times G$-action on $(G \times G) \mathcal{C}$ is free. For each $D \in \operatorname{Diag}_{G}^{\Gamma},(G \times G) \mathcal{C} \cap \ell^{2}(D)$ is a Hilbert basis of $\ell^{2}(D)$ invariant under $\operatorname{Stab}_{G \times G}(D) \subseteq G \times G$. The $\operatorname{Stab}_{G \times G}(D)$-action on $(G \times G) \mathcal{C} \cap \ell^{2}(D)$ is free. Choose a subset $\mathcal{C}_{D} \subseteq(G \times G) \mathcal{C} \cap \ell^{2}(D)$ which is a $S t a b_{G \times G}(D)$-basis of $\ell^{2}(D)$. Then

$$
\mathcal{C}^{\prime}:=\bigsqcup_{D \in \operatorname{Diag}_{G}^{\Gamma}} \mathcal{C}_{D}
$$

is a $G \times G$-basis of $\ell^{2}(\Gamma \times \Gamma)$ inscribed in $\operatorname{Diag}_{G}^{\Gamma}$.

We naturally view $\Gamma \times \Gamma$ as a subset of $\ell^{2}(\Gamma \times \Gamma)$; it is the standard Hilbert basis of $\ell^{2}(\Gamma \times \Gamma)$. A $G \times G$-basis $\mathcal{E}$ of $\ell^{2}(\Gamma \times \Gamma)$ (as defined in 4.4) will be called standard if $\mathcal{E} \subseteq \Gamma \times \Gamma$. Each standard $G \times G$-basis of $\ell^{2}(\Gamma \times \Gamma)$ is orthonormal and inscribed in $(\Gamma \times \Gamma) / \Delta$. Lemma 39 implies that there exists a standard orthonormal $G \times G$-basis of $\ell^{2}(\Gamma \times \Gamma)$ inscribed in $\operatorname{Diag}_{G}^{\Gamma}$.

We will tacitly assume that each $G \times G$-basis $\mathcal{C}$ inscribed in $\operatorname{Diag}_{G}^{\Gamma}$ is ordered, that is for each $D \in \operatorname{Diag}_{G}^{\Gamma}$, the set $(G \times G) \mathcal{C} \cap \ell^{2}(D)$ is given an ordering.

10.3. Square maps. A square map will be a generic term for $G \times G$-equivariant maps

$$
\ell^{2}(\Gamma \times \Gamma) \rightarrow \ell^{2}(\Gamma \times \Gamma) \quad \text { or } \quad H_{1}^{(2)}(\hat{S}) \rightarrow H_{1}^{(2)}(\hat{Y}) \hat{\otimes} H_{1}^{(2)}(\hat{Z}),
$$

where $G \leq \Gamma$ is a subgroup of finite index.

Let $p_{1}, p_{2}: \Gamma \times \Gamma \rightarrow \Gamma$ be the projections on the first and second coordinate, respectively. The following lemma is an easy exercise.

Lemma 40. For each diagonal $D \in(\Gamma \times \Gamma) / \Delta$, the restricted maps $p_{1}, p_{2}: D \rightarrow \Gamma$ are bijections. In particular, $p_{1}$ and $p_{2}$ induce isometric isomorphisms $p_{1}, p_{2}: \ell^{2}(D) \rightarrow \ell^{2}(\Gamma)$ of Hilbert spaces.

Let $\mathcal{C}$ be any $G \times G$-basis of $\ell^{2}(\Gamma \times \Gamma)$ inscribed in $\operatorname{Diag}_{G}^{\Gamma}$. Define a function

$$
\begin{aligned}
& \diamond_{\mathcal{C}}:(G \times G) \mathcal{C} \rightarrow \ell^{2}(\Gamma \times \Gamma) \quad \text { by } \\
& \diamond_{\mathcal{C}}(c):=p_{1}(c) \otimes p_{2}(c) \in \ell^{2}(\Gamma) \hat{\otimes} \ell^{2}(\Gamma)=\ell^{2}(\Gamma \times \Gamma), \quad c \in(G \times G) \mathcal{C} .
\end{aligned}
$$

This map commutes with the left $G \times G$-action. We would like to extend this to a map of Hilbert $G \times G$-modules $\diamond_{\mathcal{C}}: \ell^{2}(\Gamma \times \Gamma) \rightarrow \ell^{2}(\Gamma \times \Gamma)$ and call it the square map corresponding to $\mathcal{C}$. Such extension might not always be possible. One way to avoid this extension problem is to work with densely defined operators instead of bounded ones. Alternatively, one might consider modules over the von Neumann algebra $\mathcal{N}(G \times G)$ instead of Hilbert modules. We will explicitly define the extension $\diamond_{\mathcal{C}}$ in 10.5 below as the composition $\nabla_{\mathcal{C}} \circ \tau_{\mathcal{C}}^{-1}$ for some appropriately chosen $\nabla_{\mathcal{C}}$ and $\tau_{\mathcal{C}}$. 


\subsection{Glide maps.}

Definition 41. $A(\Gamma, G)$-glide map is a map $\tau: \ell^{2}(\Gamma \times \Gamma) \rightarrow \ell^{2}(\Gamma \times \Gamma)$ with the following properties.

- $\tau$ is a map of Hilbert $G \times G$-modules.

- $\tau$ preserves diagonals, i.e. for each diagonal $D \in(\Gamma \times \Gamma) / \Delta, \quad \tau\left(\ell^{2}(D)\right) \subseteq \ell^{2}(D)$ and the restricted map $\tau: \ell^{2}(D) \rightarrow \ell^{2}(D)$ is a weak isomorphism.

Let $\mathcal{E}$ be a standard $G \times G$-basis of $\ell^{2}\left(\Gamma \times \Gamma\right.$ ) inscribed in $\operatorname{Diag}_{G}^{\Gamma}$ (as in 10.2). For each (ordered) $G \times G$-basis $\mathcal{C}$ of $\ell^{2}(\Gamma \times \Gamma)$ inscribed in $\operatorname{Diag}_{G}^{\Gamma}$, define a map

$$
\tau_{\mathcal{C}}: \ell^{2}(\Gamma \times \Gamma) \rightarrow \ell^{2}(\Gamma \times \Gamma)
$$

as follows. The cardinality of a $G \times G$-basis equals $\operatorname{dim}_{G \times G} \ell^{2}(\Gamma \times \Gamma)=n^{2}$, hence $\# \mathcal{E}=n^{2}=\# \mathcal{C}$ and we can choose a bijection $\tau_{\mathcal{C}}: \mathcal{E} \rightarrow \mathcal{C}$. Moreover, for each $D \in \operatorname{Diag}_{G}^{\Gamma}, \mathcal{E} \cap \ell^{2}(D)$ is a $\operatorname{Stab}_{G \times G}(D)$-basis of $\ell^{2}(D)$, so $\#\left(\mathcal{E} \cap \ell^{2}(D)\right)=\operatorname{dim}_{S t a b_{G \times G}(D)} \ell^{2}(D)$. The same argument applies for $\mathcal{C}$ in place of $\mathcal{E}$, hence $\#\left(\mathcal{E} \cap \ell^{2}(D)\right)=\#\left(\mathcal{C} \cap \ell^{2}(D)\right)$ for each $D \in \operatorname{Diag}_{G}^{\Gamma}$. Using the order on $\mathcal{C} \cap \ell^{2}(D)$ the bijection $\tau_{\mathcal{C}}: \mathcal{E} \rightarrow \mathcal{C}$ can be chosen canonically and so that for each $e \in \mathcal{E}, e$ and $\tau(e)$ are supported on the same diagonal $D \in \operatorname{Diag}_{G}^{\Gamma}$. Next extend $\tau_{\mathcal{C}}$ to a bijective $G \times G$-map $\tau_{\mathcal{C}}:(G \times G) \mathcal{E} \rightarrow(G \times G) \mathcal{C}$ in the obvious way: $\tau_{\mathcal{C}}((g, h) c):=(g, h) \cdot\left(\tau_{\mathcal{C}}(c)\right)$. This map sends an orthonormal $G \times G$-invariant basis to a $G \times G$-invariant basis, hence extends to a map of Hilbert $G \times G$-modules $\tau_{\mathcal{C}}: \ell^{2}(\Gamma \times \Gamma) \rightarrow \ell^{2}(\Gamma \times \Gamma)$ which is a weak isomorphism.

Lemma 42. $\tau_{\mathcal{C}}: \ell^{2}(\Gamma \times \Gamma) \rightarrow \ell^{2}(\Gamma \times \Gamma)$ is a $(\Gamma, G)$-glide map.

Proof. We only need to show that $\tau_{\mathcal{C}}$ preserves diagonals. Take any diagonal $D^{\prime} \in(\Gamma \times \Gamma) / \Delta$ and $e^{\prime} \in(G \times G) \mathcal{E} \cap \ell^{2}\left(D^{\prime}\right)$. Then $e^{\prime}=(a, b) e$ for some $a, b \in \Gamma$ and $e \in \mathcal{E}$, and $e$ is supported on the diagonal $\left(a^{-1}, b^{-1}\right) D^{\prime}$, hence $\left(a^{-1}, b^{-1}\right) D^{\prime} \in \operatorname{Diag}_{G}^{\Gamma}$. By the definition of $\tau_{\mathcal{C}}, \tau_{\mathcal{C}}(e)$ is also supported on $\left(a^{-1}, b^{-1}\right) D^{\prime}$. Then the basis function

$$
\tau_{\mathcal{C}}\left(e^{\prime}\right)=\tau_{\mathcal{C}}((a, b) e)=(a, b) \cdot\left(\tau_{\mathcal{C}}(e)\right)
$$

is supported on $(a, b)\left(a^{-1}, b^{-1}\right) D^{\prime}=D^{\prime}$. This shows that $\tau_{\mathcal{C}}$ maps the orthonormal basis

$$
(G \times G) \mathcal{E} \cap \ell^{2}\left(D^{\prime}\right)
$$

of $\ell^{2}\left(D^{\prime}\right)$ to $\ell^{2}\left(D^{\prime}\right)$. Since $\tau_{\mathcal{C}}$ is a weak isomorphism, the restricted map $\tau_{\mathcal{C}}: \ell^{2}\left(D^{\prime}\right) \rightarrow \ell^{2}\left(D^{\prime}\right)$ is as well.

Conversely, each glide map $\tau$ provides a basis of $\ell^{2}(\Gamma \times \Gamma)$ inscribed in $\operatorname{Diag}_{G}^{\Gamma}$, namely $\tau(\mathcal{E})$. This means that choosing glide maps is equivalent to choosing (ordered) $G \times G$-bases inscribed in $(\Gamma \times \Gamma) / \Delta$.

10.5. Glide square maps. Fix some standard $G \times G$-basis $\mathcal{E}$ of $\ell^{2}(\Gamma \times \Gamma)$ inscribed in $(\Gamma \times \Gamma) / \Delta$ (as in 10.2). Let $\mathcal{C}$ be a $G \times G$-basis of $\ell^{2}(\Gamma \times \Gamma)$ inscribed in $(\Gamma \times \Gamma) / \Delta$ (not necessarily orthonormal) and $\tau_{\mathcal{C}}: \ell^{2}(\Gamma \times \Gamma) \rightarrow \ell^{2}(\Gamma \times \Gamma)$ be the glide map corresponding to $\mathcal{C}$. Define a function

$$
\begin{aligned}
& \diamond_{\mathcal{C}}: \Gamma \times \Gamma \rightarrow \ell^{2}(\Gamma \times \Gamma) \quad \text { by } \\
& \diamond_{\mathcal{C}}(e):=p_{1}\left(\tau_{\mathcal{C}}(e)\right) \hat{\otimes} p_{2}\left(\tau_{\mathcal{C}}(e)\right) \in \ell^{2}(\Gamma) \otimes \ell^{2}(\Gamma)=\ell^{2}(\Gamma \times \Gamma), \quad e \in \Gamma \times \Gamma .
\end{aligned}
$$


The map $\nabla_{\mathcal{C}}: \Gamma \times \Gamma \rightarrow \ell^{2}(\Gamma \times \Gamma)$ is $G \times G$-equivariant. This follows from the $G \times G$ equivariance of $\tau_{\mathcal{C}}$ : for $(g, h) \in G \times G$,

$$
\begin{aligned}
& \diamond_{\mathcal{C}}((g, h) e)=p_{1}\left(\tau_{\mathcal{C}}((g, h) e)\right) \otimes p_{2}\left(\tau_{\mathcal{C}}((g, h) e)\right) \\
& =p_{1}\left((g, h) \tau_{\mathcal{C}}(e)\right) \otimes p_{2}\left((g, h) \tau_{\mathcal{C}}(e)\right)=\left(g\left[p_{1}\left(\tau_{\mathcal{C}}(e)\right)\right]\right) \otimes\left(h\left[p_{2}\left(\tau_{\mathcal{C}}(e)\right)\right]\right) .
\end{aligned}
$$

Lemma 43. For each $e \in \mathcal{E}$, the maps $\varphi, \psi: G \rightarrow \ell^{2}(\Gamma)$ given by compositions

$$
\begin{aligned}
G \stackrel{\cong}{\longrightarrow}(G \times 1) e \stackrel{\tau_{\mathcal{C}}}{\longrightarrow} \tau_{\mathcal{C}}((G \times 1)(e))=(G \times 1) \tau_{\mathcal{C}}(e) \stackrel{p_{1}}{\longrightarrow} \ell^{2}(\Gamma), \\
G \stackrel{{ }^{\prime}}{\longrightarrow}(1 \times G) e \stackrel{\tau_{\mathcal{C}}}{\longrightarrow} \tau_{\mathcal{C}}((1 \times G)(e))=(1 \times G) \tau_{\mathcal{C}}(e) \stackrel{p_{2}}{\longrightarrow} \ell^{2}(\Gamma),
\end{aligned}
$$

respectively, are $G$-equivariant and extend to injective maps of Hilbert $G$-modules $\varphi, \psi: \ell^{2}(G) \rightarrow$ $\ell^{2}(\Gamma)$.

Proof. We prove the statement for $\varphi$. The $G$-equivariance of the compositions follows from the $G \times G$-equivariance of $\tau_{\mathcal{C}}$ and $G \times 1$-equivariance of $p_{1}$.

For distinct $g, h \in G,(g \times 1) e$ and $(h \times 1) e$ are supported on different diagonals in $(\Gamma \times \Gamma) / \Delta$ by Lemma $37(\mathrm{a})$, hence $(g \times 1) \tau_{\mathcal{C}}(e)$ and $(h \times 1) \tau_{\mathcal{C}}(e)$ are also supported on different diagonals. Therefore $(G \times 1) \tau_{\mathcal{C}}(e)$ is an orthonormal Hilbert basis of its span in $\ell^{2}(\Gamma \times \Gamma)$. This also shows that the first two maps in the composition are bijections and preserve orthogonality.

Let $D \in(\Gamma \times \Gamma) / \Delta$ be the diagonal on which $\tau_{\mathcal{C}}(e)$ is supported, then $D=(a, b) \Delta$ for some $(a, b) \in \Gamma \times \Gamma$. For each $g \in G$, let $h_{g}:=b a^{-1} g a b^{-1}$. Since $G$ is normal, $h_{g} \in G$. Also,

$$
\left(g, h_{g}\right) D=\left(g, h_{g}\right)(a, b) \Delta=\left(g a, h_{g} b\right) \Delta=\left(g a, b a^{-1} g a\right) \Delta=(a, b) \Delta=D .
$$

This shows that $\left(g, h_{g}\right) \tau_{\mathcal{C}}(e)$ is supported on $D$, hence the map

$$
\theta:(G \times 1) \tau_{\mathcal{C}}(e) \rightarrow \ell^{2}(D)
$$

is well-defined by the formula $\theta\left((g, 1) \tau_{\mathcal{C}}(e)\right):=\left(g, h_{g}\right) \tau_{\mathcal{C}}(e)$.

The diagram

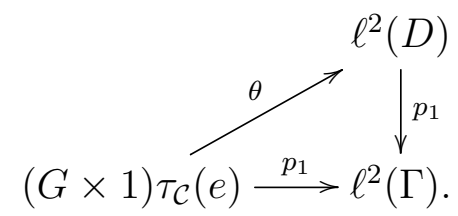

commutes because the $1 \times G$ - action on $\Gamma \times \Gamma$ does not change the first coordinate. The vertical map is an isometric isomorphism. Hence it remains to show that $\theta$ extends to a bounded injective map $\overline{\operatorname{span}}\left((G \times 1) \tau_{\mathcal{C}}\right) \rightarrow \ell^{2}(\Gamma)$.

We first show that $\theta$ is injective. Suppose $\left(g, h_{g}\right) \tau_{\mathcal{C}}(e)=\left(g^{\prime}, h_{g^{\prime}}\right) \tau_{\mathcal{C}}(e)$ for some $g, g^{\prime} \in G$. Since $\tau_{\mathcal{C}}$ is a $(\Gamma, G)$-glide map corresponding to the $G \times G$-basis $\mathcal{C}$, the set $(G \times G) \tau_{\mathcal{C}}(e)$ is a $G \times G$-invariant basis of its span, hence $\left(g, h_{g}\right)=\left(g^{\prime}, h_{g^{\prime}}\right)$, which implies $g=g^{\prime}$. Moreover, the image of $\theta$ is contained in $(G \times G) \tau_{\mathcal{C}}(e) \cap \ell^{2}(D)$, which is part of a basis (meaning a not-necessarily-orthonormal $1 \times 1$-basis) of $\ell^{2}(D)$. This implies that $\theta$ extends to a bounded injective map

$$
\theta: \overline{\operatorname{span}}\left((G \times 1) \tau_{\mathcal{C}}\right) \rightarrow \ell^{2}(\Gamma)
$$


Lemma 44. The map $\nabla_{\mathcal{c}}: \Gamma \times \Gamma \rightarrow \ell^{2}(\Gamma \times \Gamma)$ in (34) extends to a $G \times G$-Hilbert module map

$$
\nabla_{\mathcal{C}}: \ell^{2}(\Gamma \times \Gamma) \rightarrow \ell^{2}(\Gamma \times \Gamma) \text {. }
$$

For each $e \in \mathcal{E}$, the restricted map $\nabla_{\mathcal{C}}: \ell^{2}((G \times G) e) \rightarrow \ell^{2}(\Gamma \times \Gamma)$ is injective. If $\mathcal{C}$ is orthonormal, then $\nabla_{\mathcal{C}}: \ell^{2}((G \times G) e) \rightarrow \ell^{2}(\Gamma \times \Gamma)$ is an isometric isomorphism onto its image.

Proof. Since $\Gamma \times \Gamma=(G \times G) \mathcal{E}$ and $\mathcal{E}$ is finite, it suffices to show that for each $e \in \mathcal{E}$, the restricted map $\nabla_{\mathcal{C}}:(G \times G) e \rightarrow \ell^{2}(\Gamma \times \Gamma)$ extends to an injective $G \times G$-Hilbert module map

$$
\diamond_{\mathcal{C}}: \ell^{2}((G \times G) e) \rightarrow \ell^{2}(\Gamma \times \Gamma) .
$$

Let $\varphi$ and $\psi$ be the bounded injective maps from the previous lemma. If we identify $\ell^{2}((G \times G) e) \cong \ell^{2}(G) \hat{\otimes} \ell^{2}(G)$ and $\ell^{2}(\Gamma \times \Gamma) \cong \ell^{2}(\Gamma) \hat{\otimes} \ell^{2}(\Gamma)$, then the restriction of $\varphi \hat{\otimes} \psi:$ $\ell^{2}(G) \hat{\otimes} \ell^{2}(G) \rightarrow \ell^{2}(\Gamma) \hat{\otimes} \ell^{2}(\Gamma)$ to $(G \times G) e$ coincides with $\nabla_{\mathcal{C}}:(G \times G) e \rightarrow \ell^{2}(\Gamma \times \Gamma)$, so $\varphi \hat{\otimes} \psi$ is the desired bounded injective extension.

Definition 45. The map $\diamond_{\mathcal{C}}: \ell^{2}(\Gamma \times \Gamma) \rightarrow \ell^{2}(\Gamma \times \Gamma)$ in Lemma 44 will be called the $(\Gamma, G)$-glide square map corresponding to $\mathcal{C}$.

If $\diamond_{\mathcal{C}}$ is the map from (33), then the following diagram commutes.

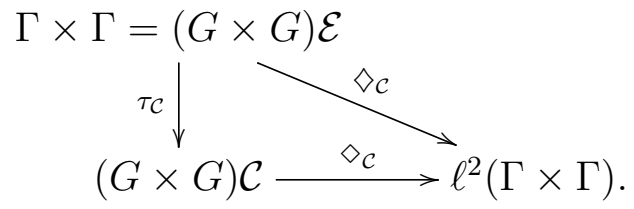

We also have the maps

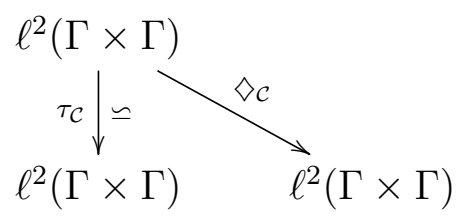

where $\simeq$ denotes a weak isomorphism.

Definition 46. The square map corresponding to $\mathcal{C}$,

$$
\diamond_{\mathcal{C}}: \ell^{2}(\Gamma \times \Gamma) \rightarrow \ell^{2}(\Gamma \times \Gamma)
$$

is the densely defined operator $\nabla_{\mathcal{C}} \circ \tau_{\mathcal{C}}^{-1}$.

We use the arrow "—" to indicate densely defined operators. Then

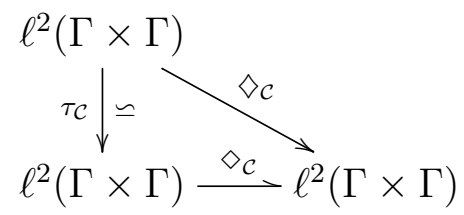

is an extension of the two previous diagrams. If $\mathcal{C}$ is orthonormal then $\diamond_{\mathcal{C}}$ is a bounded operator defined on all of $\ell^{2}(\Gamma \times \Gamma)$. 
10.6. Lifting the basis in $\hat{X}$. Consider the diagram

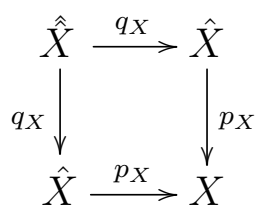

as in (7), where $X, \hat{X}$ are part of a graph system or a surface system. Each component of $\hat{X}$ is mapped by $q_{X}$ isomorphically onto $\hat{X}$. This follows from the prof of Theorem $7(\mathrm{~g})$ by replacing $Y$ with $\hat{X}$.

To distinguish between the two covers, relabel diagram (35) as

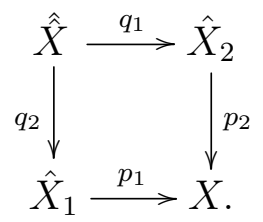

Relabel $\Gamma \times \Gamma$ as $\Gamma_{1} \times \Gamma_{2}$. Since $\operatorname{dim}_{\Gamma} \mathrm{H}(\hat{X})=1$, then $\mathrm{H}(\hat{X})$ is isometrically isomorphic to $\ell^{2}(\Gamma)$. $\mathrm{H}(\hat{X})$ has an orthonormal $\Gamma$-basis consisting of one vector. Let $e_{1}$ be the copy of this vector in $\mathrm{H}\left(\hat{X}_{1}\right)$ and $e_{2}$ its copy in $\mathrm{H}\left(\hat{X}_{2}\right)$.

Lemma 47. The lifts $q_{1}^{\uparrow}\left(\Gamma_{2} e_{2}\right)$ and $q_{2}^{\uparrow}\left(\Gamma_{1} e_{1}\right)$ coincide and $q_{1}^{\uparrow}\left(\Gamma_{2} e_{2}\right)$ is an orthonormal $\Gamma \times \Gamma$ invariant basis of $\mathrm{H}(\hat{X})$.

Proof. Each component of $\hat{X}$ is mapped by $q_{2}$ isomorphically onto $\hat{X}$, and similarly for $q_{1}$. This implies $q_{1}^{\uparrow}\left(\Gamma_{2} e_{2}\right)=q_{2}^{\uparrow}\left(\Gamma_{1} e_{1}\right)$.

The $\Gamma_{i}$-action on $\hat{X}_{i}$ induces a $\Gamma_{i}$-action on $\hat{X}$. This induces a $\Gamma_{1} \times \Gamma_{2}$-action on $\hat{X}$. The $\Gamma_{i}$-action on $\Gamma_{i} e_{i}$ is free and transitive because $\left\{e_{i}\right\}$ is a $\Gamma_{i}$-basis. Hence $\Gamma_{i}$ acts on the lift $q_{1}^{\uparrow}\left(\Gamma_{2} e_{2}\right)=q_{2}^{\uparrow}\left(\Gamma_{1} e_{1}\right)$ and the induced $\Gamma_{1} \times \Gamma_{2}$-action on this lift is free and transitive.

Denote

$$
\overline{\mathcal{E}}:=q_{1}^{\uparrow}\left(\Gamma_{2} e_{2}\right)=q_{2}^{\uparrow}\left(\Gamma_{1} e_{1}\right) .
$$

$\overline{\mathcal{E}}$ is a $\Gamma \times \Gamma$-invariant orthonormal basis of $\mathrm{H}(\hat{X})$ inscribed in $\operatorname{Comp}(\hat{X})$.

Lemma 48. For graph systems and surface systems, the map $\eta: \mathrm{H}(\hat{X}) \rightarrow \mathrm{H}(\hat{X}) \hat{\otimes} \mathrm{H}(\hat{X})$ defined by $\eta(e):=q_{1}(e) \otimes q_{2}(e)$ for $e \in \overline{\mathcal{E}}$ is an isometric isomorphism of Hilbert $\Gamma \times \Gamma$-modules. In particular, $\mathrm{H}(\hat{X})$ is isometrically isomorphic to $\ell^{2}(\Gamma \times \Gamma)$ and $\eta$ is the same as the standard isomorphism $\ell^{2}(\Gamma \times \Gamma) \rightarrow \ell^{2}(\Gamma) \hat{\otimes} \ell^{2}(\Gamma)$.

Proof. Immediate from the proof of Lemma 47.

Lemma 49. Let $\mathcal{S}^{\circ}$ be a symmetric graph system or a symmetric surface system. The map $\eta$ from Lemma 48 induces a map $\xi: \mathrm{H}\left(\hat{S}^{\circ}\right) \rightarrow \mathrm{H}\left(\hat{Y}^{\circ}\right) \hat{\otimes} \mathrm{H}\left(\hat{Z}^{\circ}\right)$ which is an isometric isomorphism of Hilbert $\Gamma \times \Gamma$-modules. In particular,

$$
\operatorname{dim}_{\Gamma \times \Gamma} \mathrm{H}\left(\hat{S}^{\circ}\right)=\operatorname{dim}_{\Gamma \times \Gamma} \mathrm{H}\left(\hat{Y}^{\circ}\right) \hat{\otimes} \mathrm{H}\left(\hat{Z}^{\circ}\right)=\operatorname{dim}_{\Gamma} \mathrm{H}\left(\hat{Y}^{\circ}\right) \cdot \operatorname{dim}_{\Gamma} \mathrm{H}\left(\hat{Z}^{\circ}\right) .
$$


Proof. Since $\alpha: Y^{\circ} \rightarrow X$ and $\beta: Z^{\circ} \rightarrow X$ are covers, then by Theorem $7(\mathrm{~g}), \hat{\alpha}$ and $\hat{\beta}$ map the components of $\hat{Y}^{\circ}$ and $\hat{Z}^{\circ}$ isomorphically onto $\hat{X}$. Then for each $K^{\circ} \in \operatorname{Comp}\left(\hat{Y}^{\circ}\right)$ and $L^{\circ} \in \operatorname{Comp}\left(\hat{Z}^{\circ}\right)$ the restricted maps $\bar{\alpha}: K^{\circ} \rightarrow X$ and $\bar{\beta}: L^{\circ} \rightarrow X$ are the same as $p_{X}: \hat{X} \rightarrow X$, and the diagram

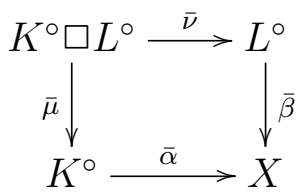

is isomorphic to (35). The explicit isomorphism is given by the map $\Theta$ in (10). As in Lemma 48, it follows that the map $\xi: \mathrm{H}\left(K^{\circ} \square L^{\circ}\right) \rightarrow \mathrm{H}\left(K^{\circ}\right) \hat{\otimes} \mathrm{H}\left(L^{\circ}\right)$ is the same as the standard isomorphism $\ell^{2}(\Gamma \times \Gamma) \rightarrow \ell^{2}(\Gamma) \hat{\otimes} \ell^{2}(\Gamma)$, only here it is a $G \times G$-module map rather than a $\Gamma \times \Gamma$-module map. Now combine all such $G \times G$-isomorphisms for all pairs $\left(K^{\circ}, L^{\circ}\right) \in \operatorname{Comp}\left(\hat{Y}^{\circ}\right) \times \operatorname{Comp}\left(\hat{Z}^{\circ}\right)$ into one $\Gamma \times \Gamma$-isomorphism $\xi: \mathrm{H}\left(\hat{\hat{S}}^{\circ}\right) \rightarrow \mathrm{H}\left(\hat{Y}^{\circ}\right) \hat{\otimes} \mathrm{H}\left(\hat{Z}^{\circ}\right)$.

10.7. Bases and maps coming from systems. Recall that an orthonormal $G \times G$-subbasis of $\ell^{2}(\Gamma \times \Gamma)$ is a subset of some orthonormal $G \times G$-basis. If $\mathcal{C}$ is a $G \times G$-subbasis inscribed in $\operatorname{Diag}_{G}^{\Gamma}$, we define the square map $\diamond_{\mathcal{C}}$ corresponding to $\mathcal{C}$ by extending $\mathcal{C}$ to a $G \times G$-basis $\mathcal{C}^{\prime}$ inscribed in $\operatorname{Diag}_{G}^{\Gamma}$, defining $\diamond_{\mathcal{C}^{\prime}}$ as in Definition 46, and then restricting $\diamond_{\mathcal{C}^{\prime}}$ to $\overline{\operatorname{span}}((G \times G) \mathcal{C})$. Since $\diamond_{\mathcal{C}^{\prime}}$ is densely defined, $\diamond_{\mathcal{C}}$ is defined on a dense subset of $\overline{\operatorname{span}}((G \times G) \mathcal{C})$.

Each symmetric pair of graph or surface systems $\left(\mathcal{S}^{\circ}, \mathcal{S}\right.$ ) (as in 3.6 or 8.1) gives rise to square maps as follows. Let $G$ be the common stabilizer of the components in $\hat{Y}^{\circ}$ and in $\hat{Z}^{\circ}$. Fix any $K^{\circ} \in \operatorname{Comp}\left(\hat{Y}^{\circ}\right)$ and $L^{\circ} \in \operatorname{Comp}\left(\hat{Z}^{\circ}\right)$ and let

$$
K:=K^{\circ} \cap \hat{Y} \quad \text { and } \quad L:=L^{\circ} \cap \hat{Z},
$$

then $K \square L \subseteq K^{\circ} \square L^{\circ} \subseteq \hat{S}^{\circ}$. Let

$$
U:=\mathrm{H}(K \square L) \subseteq \mathrm{H}\left(K^{\circ} \square L^{\circ}\right) \cong \ell^{2}(\Gamma \times \Gamma) .
$$

$U$ is a Hilbert $G \times G$-module because $K$ and $L$ are $G$-invariant.

By Lemma $4, \hat{S}$ is a $\Delta$-invariant subgraph/subsurface of $\hat{\hat{S}}$ and $\hat{S}^{\circ}$ is a $\Delta$-invariant subgraph/subsurface of $\hat{S}^{\circ}$. The following theorem is proved in exactly the same way as Theorem 30 by replacing $\hat{\beta}: \hat{Z} \rightarrow \hat{X}$ with $\hat{\beta} \circ \hat{\nu}: \hat{S} \rightarrow \hat{X}, \hat{\beta}: \hat{Z}^{\circ} \rightarrow \hat{X}$ with $\hat{\beta} \circ \hat{\nu}: \hat{S}^{\circ} \rightarrow \hat{X}$, and $\Gamma$ with $\Delta$.

Theorem 50. For any graph system there exist orthonormal $\Delta$-bases $\mathcal{C}$ of $\mathrm{H}(\hat{S})$ and $\mathcal{C}^{\circ}$ of $\mathrm{H}\left(\hat{S}^{\circ}\right)$ such that

(1) $\mathcal{C} \subseteq \mathcal{C}^{\circ}$

(2) $\mathcal{C}$ is inscribed in $\operatorname{Comp}(\hat{S})$,

(3) $\mathcal{C}^{\circ}$ is inscribed in $\operatorname{Comp}\left(\hat{S}^{\circ}\right)$, and

(4) for each $c \in \mathcal{C}^{\circ}$, the restricted map $\hat{\beta} \circ \hat{\nu}: \overline{\operatorname{span}}(\Delta c) \rightarrow \mathrm{H}(\hat{X})$ is a weak isomorphism. 
10.8. From diagonal to square. Assume that $\left(\mathcal{S}^{\circ}, \mathcal{S}\right)$ is a symmetric pair of graph systems. By Lemma 9,

$$
\hat{S}=\operatorname{Ind}_{\Delta}^{\Gamma \times \Gamma} \hat{S}, \quad \hat{S}^{\circ}=\operatorname{Ind}_{\Delta}^{\Gamma \times \Gamma} \hat{S}^{\circ} .
$$

This implies that the $\Delta$-basis $\mathcal{C}^{\circ}$ of $\mathrm{H}\left(\hat{S}^{\circ}\right)$ from Theorem 50 is also a $\Gamma \times \Gamma$-basis of $\mathrm{H}\left(\hat{S}^{\circ}\right)$. As before, fix $K^{\circ} \in \operatorname{Comp}\left(\hat{Y}^{\circ}\right)$ and $L^{\circ} \in \operatorname{Comp}\left(\hat{Z}^{\circ}\right)$ and denote $K:=K^{\circ} \cap \hat{Y}, L:=L^{\circ} \cap \hat{Z}$. By applying elements of $\Gamma \times \Gamma$ we can assume that $\mathcal{C}^{\circ}$ is inscribed in $\operatorname{Comp}\left(K^{\circ} \square L^{\circ}\right)$ and $\mathcal{C}$ is inscribed in $\operatorname{Comp}(K \square L)$. Identify $\mathrm{H}\left(K^{\circ} \square L^{\circ}\right)$ with $\ell^{2}(\Gamma \times \Gamma)$ as in Lemma 49. By applying elements of $G \times G$ we can further assume that $\mathcal{C}^{\circ}$ is inscribed in $\operatorname{Diag}_{G}^{\Gamma}$.

This argument shows that for symmetric pairs $\left(\mathcal{S}^{\circ}, \mathcal{S}\right.$ ) (as in 6.2) of graph systems, $\Delta$-bases of $\mathrm{H}(\hat{S})$ inscribed in $\operatorname{Comp}(\hat{S})$ give rise to $G \times G$-bases of $\mathrm{H}(K \square L) \subseteq \ell^{2}(\Gamma \times \Gamma)$ inscribed in $\operatorname{Diag}_{G}^{\Gamma}$, and therefore to square maps $\diamond: \mathrm{H}(K \square L) \rightarrow \mathrm{H}(K) \otimes \mathrm{H}(L)$.

Theorem 51. Suppose $U$ is the Hilbert $G \times G$-submodule of $\ell^{2}(\Gamma \times \Gamma)$ coming from a symmetric pair of graph systems $\left(\mathcal{S}^{\circ}, \mathcal{S}\right)$ as in 10.7. Suppose that there exist a subgroup $H$ of finite index in $G$ and an $H \times H$-basis $\mathcal{C}$ of $U$ inscribed in $\operatorname{Diag}_{H}^{\Gamma}$ such that the corresponding square map $\diamond_{\mathcal{C}}: U \rightarrow \ell^{2}(\Gamma \times \Gamma)$ is injective. Then $S H N C$ holds for $\mathcal{S}$.

Proof. The square map $\diamond_{\mathcal{C}}: U \rightarrow \ell^{2}(\Gamma \times \Gamma)$ is $H \times H$-equivariant. Using diagram (36) the square map can be equivalently written as

$$
\mathrm{H}(K \square L) \stackrel{\diamond_{\mathcal{C}}}{\longrightarrow} \mathrm{H}(K) \hat{\otimes} \mathrm{H}(L)
$$

Since this map is injective, it induces an injective $H \times H$-map on the finite sums

$$
\mathrm{H}(\hat{Y} \square \hat{Z})=\bigoplus_{\left(K^{\circ}, L^{\circ}\right)} \mathrm{H}(K \square L) \longrightarrow \bigoplus_{\left(K^{\circ}, L^{\circ}\right)} \mathrm{H}(K) \hat{\otimes} \mathrm{H}(L)=\mathrm{H}(\hat{Y}) \hat{\otimes} \mathrm{H}(\hat{Z}) .
$$

where $\left(K^{\circ}, L^{\circ}\right) \in \operatorname{Comp}\left(\hat{Y}^{\circ}\right) \times \operatorname{Comp}\left(\hat{Z}^{\circ}\right)$. This shows that

$$
\begin{aligned}
& \operatorname{dim}_{\Gamma \times \Gamma} \mathrm{H}(\hat{S})=\operatorname{dim}_{\Gamma \times \Gamma} \mathrm{H}(\hat{Y} \square \hat{Z}) \\
& =\frac{\operatorname{dim}_{H \times H} \mathrm{H}(\hat{Y} \square \hat{Z})}{[\Gamma \times \Gamma: H \times H]} \leq \frac{\operatorname{dim}_{H \times H} \mathrm{H}(\hat{Y}) \hat{\otimes} \mathrm{H}(\hat{Z})}{[\Gamma \times \Gamma: H \times H]} \\
& =\operatorname{dim}_{\Gamma \times \Gamma} \mathrm{H}(\hat{Y}) \hat{\otimes} \mathrm{H}(\hat{Z})=\operatorname{dim}_{\Gamma} \mathrm{H}(\hat{Y}) \cdot \operatorname{dim}_{\Gamma} \mathrm{H}(\hat{Z}),
\end{aligned}
$$

which implies SHNC by Theorem 23(d).

Remark. Theorem 51 holds under the weaker assumption that there exist a sequence $H_{i}$ of subgroups of finite index in $\Gamma$ and $H_{i} \times H_{i}$-bases $\mathcal{C}_{i}$ of $U$ inscribed in $\operatorname{Diag}_{H_{i}}^{\Gamma}$ such that the corresponding square map becomes "more and more injective", i.e.

$$
\lim _{i \rightarrow \infty} \frac{\operatorname{dim}_{H_{i} \times H_{i}} \operatorname{Ker} \diamond_{\mathcal{C}_{i}}}{\left[\Gamma \times \Gamma: H_{i} \times H_{i}\right]}=0 .
$$


10.9. Classes of groups: $\mathcal{P}$ and $\mathcal{P}_{\text {lim }}$. Let $\mathcal{P}$ be the class of groups $\Gamma$ satisfying the following condition.

\section{Condition 1. If}

- $G$ is a normal subgroup of finite index in $\Gamma$,

- $U$ is a Hilbert $G \times G$-submodule of $\ell^{2}(\Gamma \times \Gamma)$,

- there exists an orthonormal $G \times G$-basis $\mathcal{B}^{\prime}$ of $U$ inscribed in $\operatorname{Diag}_{G}^{\Gamma}$,

- there exists an orthonormal $G \times G$-basis $\mathcal{B}^{\prime \prime}$ of $U^{\perp}$ inscribed in $\operatorname{Diag}_{G}^{\Gamma}$, and

- for each $b \in \mathcal{B}^{\prime} \cup \mathcal{B}^{\prime \prime}$, the singleton $\{b\}$ is a (not necessarily orthonormal) $\Gamma \times \Gamma$-basis of $\ell^{2}(\Gamma \times \Gamma)$ (as defined in 4.4),

then

- there exists a normal subgroup $H$ of finite index in $\Gamma$ and

- there exists an $H \times H$-basis $\mathcal{C}^{\prime}$ of $U$ inscribed in $\operatorname{Diag}_{H}^{\Gamma}$

such that the corresponding square map $\diamond_{\mathcal{C}^{\prime}}: U \rightarrow \ell^{2}(\Gamma \times \Gamma)$ is injective.

For example, all infinite simple groups belong to $\mathcal{P}$.

Another class of groups, $\mathcal{P}_{\text {lim }}$, is defined by replacing the conclusion of Condition 1 by the weaker property as in the above remark: there exist a sequence $H_{i}$ of subgroups of finite index in $\Gamma$ and $H_{i} \times H_{i}$-bases $\mathcal{C}_{i}^{\prime}$ of $U$ inscribed in $\operatorname{Diag}_{H_{i}}^{\Gamma}$ such that $\left(\operatorname{dim}_{H_{i} \times H_{i}} \operatorname{Ker} \diamond_{\mathcal{C}_{i}^{\prime}}\right) /\left[\Gamma \times \Gamma: H_{i} \times H_{i}\right]$ converges to 0 .

Clearly, $\mathcal{P} \subseteq \mathcal{P}_{\text {lim }}$. With some work it is possible to show that the space $U$ coming from a pair of graph systems as in 10.7 satisfies the assumptions of Condition 1; we skip the details. It is therefore an interesting question, what groups belong to $\mathcal{P}$ or to $\mathcal{P}_{\text {lim }}$. Theorem 51 implies that if $\mathcal{P}$ (or $\mathcal{P}_{\text {lim }}$ ) contains the free group of rank 2 then SHNC holds.

10.10. Atomic square maps. Let $\left(\mathcal{S}^{\circ}, \mathcal{S}\right)$ be a symmetric pair of graph or surface systems. Suppose $\hat{\varphi}: \hat{S}^{\bullet} \rightarrow \hat{S}$ is an atomic decomposition of $\hat{S}$ as in 5.5 or 8.4.

Each such atomic decompositions yields a $\Delta$-basis $\mathcal{C}$ of $\mathrm{H}(\hat{S})$ as follows. For each $i$, choose any $M_{i} \in \operatorname{Comp}\left(\hat{S}_{i}^{\bullet}\right)$ and let $\Delta_{i}:=\operatorname{Stab} b_{\Delta}\left(M_{i}\right)$. Then $\Delta_{i}$ is free, hence ICC. Since

$$
\operatorname{dim}_{\Delta_{i}} \mathrm{H}\left(M_{i}\right)=\operatorname{dim}_{\Delta} \mathrm{H}\left(\hat{S}_{i}^{\bullet}\right)=\bar{r}\left(S_{i}^{\bullet}\right)=1,
$$

$\mathrm{H}\left(M_{i}\right)$ is isometrically isomorphic to $\ell^{2}\left(\Delta_{i}\right)$, so we can choose a vector in $\mathrm{H}\left(M_{i}\right)$ that forms an orthonormal $\Delta_{i}$-basis of $\mathrm{H}\left(M_{i}\right)$. This vector also forms an orthonormal $\Delta$-basis of $\mathrm{H}\left(\hat{S}_{i}^{\bullet}\right)$. Combining such vectors gives an orthonormal $\Delta$-basis $\overline{\mathcal{C}}$ of $\mathrm{H}\left(\hat{S}^{\bullet}\right)$ inscribed in $\operatorname{Comp}\left(\hat{S}^{\bullet}\right)$. Since $\hat{\varphi}: \hat{S}^{\bullet} \rightarrow \hat{S}$ is a weak isomorphism, $\mathcal{C}:=\hat{\varphi}(\overline{\mathcal{C}})$ is a $\Delta$-basis inscribed in $\operatorname{Comp}(\hat{S})$.

Going from diagonal to square as in 10.8 gives rise to a $G \times G$-basis $\mathcal{C}$ of $\ell^{2}(\Gamma \times \Gamma)$ inscribed in $\operatorname{Diag}_{G}^{\Gamma}$ which we call an atomic basis, and to the corresponding square map $\diamond_{\mathcal{C}}$ which we call an atomic square map.

Theorem 52. If a $(\Gamma \times \Gamma$-invariant $)$ atomic decomposition of $\hat{\hat{S}}$ can be chosen so that the corresponding atomic square map $\diamond_{\mathcal{C}}: \ell^{2}(\Gamma \times \Gamma) \rightarrow \ell^{2}(\Gamma \times \Gamma)$ (or equivalently, atomic glide square map $\diamond)$ is injective, then $S H N C$ holds for $\mathcal{S}$. 
The proof is the same as in Theorem 51, and one can similarly state a limit version of Theorem 52: assume that there is a sequence of atomic decompositions, the $i$ th of which is $H_{i} \times H_{i}$-invariant, and that the corresponding atomic square maps become "more and more injective".

\section{SubspaCES AND ARRANGEMENTS.}

For this section we will assume that $\Gamma$ is a group, $G$ is a normal subgroup of $\Gamma$ of finite index, and denote $\bar{\Gamma}:=\Gamma / G, n:=|\bar{\Gamma}|$.

11.1. G-subspaces. For a Hilbert $\Gamma$-module $H$, let

- $\mathcal{S P}(H)$ be the set of Hilbert subspaces in $H$, and

- $\mathcal{S P}(H)^{G}$ be the set of $G$-invariant Hilbert subspaces in $H$.

We will be primarily interested in the case $H:=\ell^{2}(\Gamma)$. The elements of $\mathcal{S P}(H)^{G}$ will be called $G$-subspaces. The left action of $\Gamma$ on $H$ induces $\Gamma$-actions on $\mathcal{S P}(H)$ and on $\mathcal{S P}(H)^{G}$. This in turn induces a $\bar{\Gamma}$-action on $\mathcal{S P}(H)^{G}$.

\subsection{Arrangements.}

Definition 53. Let $H$ be a Hilbert space. An arrangement in $H$ is a function $\Phi: I \rightarrow \mathcal{S P}(H)$, where $I$ is a set.

For $i \in I, \Phi(i)$ will be denoted by $U_{i}$, so an arrangement is simply an indexed family of subspaces $U_{i} \subseteq U$, possibly with repetitions.

The arrangements in $H$ form a category $\mathcal{A R \mathcal { R }}(H)$ : the morphisms from arrangement $\Phi$ : $I \rightarrow \mathcal{S P}(H)$ to arrangement $\Psi: J \rightarrow \mathcal{S P}(H)$ are the maps $f: I \rightarrow J$ such that $\Phi=\Psi \circ f$.

Definition 54. $A(\Gamma, G)$-arrangement is a function $\Phi: I \rightarrow \mathcal{S P}\left(\ell^{2}(\Gamma)\right)^{G}$ such that

- I is a free $\bar{\Gamma}$-set,

- $\Phi$ commutes with the $\bar{\Gamma}$-actions on $I$ and $\mathcal{S P}\left(\ell^{2}(\Gamma)\right)^{G}$.

The $(\Gamma, G)$-arrangements form a subcategory $\mathcal{A R \mathcal { R }}(\Gamma, G)$ of $\mathcal{A R \mathcal { R }}\left(\ell^{2}(\Gamma)\right)$ : the morphisms from $\Phi: I \rightarrow \mathcal{S P}\left(\ell^{2}(\Gamma)\right)^{G}$ to $\Psi: J \rightarrow \mathcal{S P}\left(\ell^{2}(\Gamma)\right)^{G}$ are the $\bar{\Gamma}$-equivariant maps $f: I \rightarrow J$ such that $\Phi=\Psi \circ f$.

Definition 55. The product of two $(\Gamma, G)$-arrangements $\Phi: I \rightarrow \mathcal{S P}\left(\ell^{2}(\Gamma)\right)^{G}$ and $\Psi: J \rightarrow$ $\mathcal{S P}\left(\ell^{2}(\Gamma)\right)^{G}$ is the $(\Gamma, G)$-arrangement $\Phi \times \Psi: I \times J \rightarrow \mathcal{S P}\left(\ell^{2}(\Gamma)\right)^{G}$ defined by

$$
(\Phi \times \Psi)(i, j):=\Phi(i) \cap \Psi(j)
$$

where $\bar{\Gamma}$ acts on $I \times J$ diagonally.

11.3. Arrangements coming from systems. Let $\left(\mathcal{S}^{\circ}, \mathcal{S}\right)$ be a connected symmetric pair of either graph systems as in 6.2 or surface systems as in 8.1 . Then $\mathrm{H}(\hat{X})$ has dimension 1 over $\Gamma$ and therefore is isometrically isomorphic to $\ell^{2}(\Gamma)$.

Let $I^{\circ}:=\operatorname{Comp}\left(\hat{Y}^{\circ}\right)$ and $J^{\circ}:=\operatorname{Comp}\left(\hat{Z}^{\circ}\right)$, then $I^{\circ}$ and $J^{\circ}$ are $\Gamma$-sets such that for all $K \in I^{\circ}$ and $L \in J^{\circ}, \operatorname{Stab}_{\Gamma}(K)=S t a b_{\Gamma}(L)=G$, i.e. $G$ fixes $I^{\circ}$ and $J^{\circ}$ pointwise. Therefore 
the $\Gamma$-actions on $I^{\circ}$ and $J^{\circ}$ pass to actions by the finite quotient $\bar{\Gamma}$ on $I^{\circ}$ and $J^{\circ}$, respectively. By Lemma $19, I^{\circ}$ and $J^{\circ}$ are free transitive $\bar{\Gamma}$-sets. The maps

$$
\begin{array}{ll}
\Phi_{Y}: I^{\circ} \rightarrow \mathcal{S P}\left(\ell^{2}(\Gamma)\right)^{G}, & K^{\circ} \mapsto \mathrm{H}\left(\hat{\alpha}\left(K^{\circ} \cap \hat{Y}\right)\right)=\hat{\alpha}\left(\mathrm{H}\left(K^{\circ} \cap \hat{Y}\right)\right) \quad \text { and } \\
\Phi_{Z}: J^{\circ} \rightarrow \mathcal{S P}\left(\ell^{2}(\Gamma)\right)^{G}, & L^{\circ} \mapsto \mathrm{H}\left(\hat{\beta}\left(L^{\circ} \cap \hat{Z}\right)\right)=\hat{\beta}\left(\mathrm{H}\left(L^{\circ} \cap \hat{Z}\right)\right)
\end{array}
$$

are $(\Gamma, G)$-arrangements. The $(\Gamma, G)$-arrangement $\Phi_{S}$ coming from $\hat{S} \rightarrow \hat{X}$ is the same as the product $\Phi_{Y} \times \Phi_{Z}$.

11.4. Subspaces coming from systems. If $\left(\mathcal{S}^{\circ}, \mathcal{S}\right)$ is a connected symmetric pair of graph or surface systems, pick some $K^{\circ} \in \operatorname{Comp}\left(\hat{Y}^{\circ}\right), L^{\circ} \in \operatorname{Comp}\left(\hat{Z}^{\circ}\right)$ and define

$$
\begin{aligned}
& K:=K^{\circ} \cap \hat{Y}, \quad L:=L^{\circ} \cap \hat{Z}, \\
& V:=\mathrm{H}(\hat{\alpha}(K)), \quad W:=\mathrm{H}(\hat{\beta}(L)) .
\end{aligned}
$$

If $V$ and $W$ are defined as above, we will say that $V$ and $W$ are subspaces coming from $\left(\mathcal{S}^{\circ}, \mathcal{S}\right)$.

Theorem 56. SHNC is equivalent to the following statement. If $\left(\mathcal{S}^{\circ}, \mathcal{S}\right)$ is a connected symmetric pair of graph systems as in 3.7 and 6.2 , and $V$ and $W$ come from $\left(\mathcal{S}^{\circ}, \mathcal{S}\right)$, then the following inequality holds.

$$
\mathcal{I}(V, W): \quad \sum_{\bar{g} \in \bar{\Gamma}} \operatorname{dim}_{G}(V \cap \bar{g} W) \leq \operatorname{dim}_{G} V \cdot \operatorname{dim}_{G} W .
$$

Proof.

$$
\begin{aligned}
& \operatorname{dim}_{G} V=\operatorname{dim}_{G} \mathrm{H}\left(\hat{\alpha}\left(K^{\circ} \cap \hat{Y}\right)\right)=\operatorname{dim}_{G} \mathrm{H}\left(K^{\circ} \cap \hat{Y}\right) \\
& =\operatorname{dim}_{\Gamma} \operatorname{Ind}_{G}^{\Gamma} \mathrm{H}\left(K^{\circ} \cap \hat{Y}\right)=\operatorname{dim}_{\Gamma} \mathrm{H}(\hat{Y})=b_{1}^{(2)}(\hat{Y} ; \Gamma) .
\end{aligned}
$$

Similarly, $\operatorname{dim}_{G} W=b_{1}^{(2)}(\hat{Z} ; \Gamma)$.

$$
\begin{aligned}
& \sum_{\bar{g} \in \bar{\Gamma}} \operatorname{dim}_{G}(V \cap \bar{g} W)=\sum_{\bar{g} \in \bar{\Gamma}} \operatorname{dim}_{G} \mathrm{H}\left(\hat{\alpha}\left(K^{\circ} \cap \hat{Y}\right)\right) \cap \mathrm{H}\left(\hat{\beta}\left(\bar{g}\left(L^{\circ} \cap \hat{Z}\right)\right)\right) \\
& \left.=\sum_{\bar{g} \in \bar{\Gamma}} \operatorname{dim}_{G} \mathrm{H}\left(\hat{\alpha}\left(K^{\circ} \cap \hat{Y}\right)\right) \cap \hat{\beta}\left(\bar{g}\left(L^{\circ} \cap \hat{Z}\right)\right)\right) \\
& =\sum_{\bar{g} \in \bar{\Gamma}} \operatorname{dim}_{G} \mathrm{H}\left(\left(K^{\circ} \cap \hat{Y}\right) \hat{\square}\left(\bar{g}\left(L^{\circ} \cap \hat{Z}\right)\right)\right. \\
& =\frac{1}{n} \sum_{\bar{h} \in \bar{\Gamma}} \sum_{\bar{g} \in \bar{\Gamma}} \operatorname{dim}_{G} \mathrm{H}\left(\bar{h}\left(K^{\circ} \cap \hat{Y}\right) \hat{\square}\left(\bar{g}\left(L^{\circ} \cap \hat{Z}\right)\right)\right. \\
& =\frac{1}{n} \operatorname{dim}_{G} \bigoplus_{(\bar{h}, \bar{g}) \in \bar{\Gamma} \times \bar{\Gamma}} \mathrm{H}\left(\bar{h}\left(K^{\circ} \cap \hat{Y}\right) \hat{\square}\left(\bar{g}\left(L^{\circ} \cap \hat{Z}\right)\right)\right. \\
& =\frac{1}{n} \operatorname{dim}_{G} \mathrm{H}(\hat{S})=\operatorname{dim}_{\Gamma} \mathrm{H}(\hat{S})=b_{1}^{(2)}(\hat{S} ; \Gamma) .
\end{aligned}
$$

The equivalence to SHNC now follows from Theorem 23(d'). 
11.5. Duality. Given $V, W \in \mathcal{S P}\left(\ell^{2}(\Gamma)\right)^{G}$, we will use the following notations.

$$
n:=|\bar{\Gamma}|=\operatorname{dim}_{G} \ell^{2}(\Gamma), \quad k:=\operatorname{dim}_{G} V, \quad l:=\operatorname{dim}_{G} W .
$$

The next lemma establishes a certain kind of duality in inequality $\mathcal{I}(V, W)$ between the subspaces and their orthogonal complements.

Lemma 57 (duality). If $V, W \in \mathcal{S P}\left(\ell^{2}(\Gamma)\right)^{G}$, then

$$
\begin{aligned}
& \operatorname{dim}_{G} V \cdot \operatorname{dim}_{G} W-\sum_{\bar{g} \in \bar{\Gamma}} \operatorname{dim}_{G}(V \cap \bar{g} W) \\
& =\operatorname{dim}_{G} V^{\perp} \cdot \operatorname{dim}_{G} W^{\perp}-\sum_{\bar{g} \in \bar{\Gamma}} \operatorname{dim}_{G}\left(V^{\perp} \cap \bar{g} W^{\perp}\right) .
\end{aligned}
$$

Proof. By the additivity of dimension, for each $\bar{g} \in \bar{\Gamma}$,

$$
\begin{aligned}
& \operatorname{dim}_{G}(V \cap \bar{g} W)=n-\operatorname{dim}_{G}(V \cap \bar{g} W)^{\perp} \\
& =n-\operatorname{dim}_{G} \overline{V^{\perp}+\bar{g} W^{\perp}} \\
& =n-\operatorname{dim}_{G} V^{\perp}-\operatorname{dim}_{G} \bar{g} W^{\perp}+\operatorname{dim}_{G}\left(V^{\perp} \cap \bar{g} W^{\perp}\right) \\
& =n-(n-k)-(n-l)+\operatorname{dim}_{G}\left(V^{\perp} \cap \bar{g} W^{\perp}\right) \\
& =-n+k+l+\operatorname{dim}_{G}\left(V^{\perp} \cap \bar{g} W^{\perp}\right),
\end{aligned}
$$

then

$$
\begin{aligned}
& k l-\sum_{\bar{g} \in \bar{\Gamma}} \operatorname{dim}_{G}(V \cap \bar{g} W) \\
& =k l+\sum_{\bar{g} \in \bar{\Gamma}}\left[n-k-l-\operatorname{dim}_{G}\left(V^{\perp} \cap \bar{g} W^{\perp}\right)\right] \\
& =k l+n(n-k-l)-\sum_{\bar{g} \in \bar{\Gamma}} \operatorname{dim}_{G}\left(V^{\perp} \cap \bar{g} W^{\perp}\right) \\
& =(n-k)(n-l)-\sum_{\bar{g} \in \bar{\Gamma}} \operatorname{dim}_{G}\left(V^{\perp} \cap \bar{g} W^{\perp}\right)
\end{aligned}
$$

proves the lemma.

For Hilbert subspaces $V$ and $W$ of $U, \operatorname{pr}[V, W]$ will denote the image of the orthogonal projection $\operatorname{pr}[\cdot, W]: V \rightarrow W$. We present some more duality statements. 
Lemma 58. The following inequalities are pairwise equivalent for $V, W \in \mathcal{S P}\left(\ell^{2}(\Gamma)\right)^{G}$.

$$
\begin{array}{ll}
\mathcal{I}(V, W): & \sum_{\bar{g} \in \bar{\Gamma}} \operatorname{dim}_{G}(V \cap \bar{g} W) \leq \operatorname{dim}_{G} V \cdot \operatorname{dim}_{G} W \\
\mathcal{I}_{1}(V, W): & \sum_{\bar{g} \in \bar{\Gamma}} \operatorname{dim}_{G}\left(V^{\perp} \cap \bar{g} W^{\perp}\right) \leq \operatorname{dim}_{G} V^{\perp} \cdot \operatorname{dim}_{G} W^{\perp} \\
\mathcal{I}_{2}(V, W): & \sum_{\bar{g} \in \bar{\Gamma}} \operatorname{dim}_{G}\left(p r\left[V, \bar{g} W^{\perp}\right]\right) \geq \operatorname{dim}_{G} V \cdot \operatorname{dim}_{G} W^{\perp} \\
\mathcal{I}_{3}(V, W): & \sum_{\bar{g} \in \bar{\Gamma}} \operatorname{dim}_{G}\left(p r\left[\bar{g} V, W^{\perp}\right]\right) \geq \operatorname{dim}_{G} V \cdot \operatorname{dim}_{G} W^{\perp} \\
\mathcal{I}_{4}(V, W): & \frac{\sum_{\bar{g} \in \bar{\Gamma}} \operatorname{dim}_{G}(V \cap \bar{g} W)}{\operatorname{dim}_{G} V} \leq \frac{\sum_{\bar{g} \in \bar{\Gamma}} \operatorname{dim}_{G} \bar{g} W}{\operatorname{dim}_{G} \ell^{2}(\Gamma)} \text { if } V \neq 0 \\
\mathcal{I}_{5}(V, W): & \sum_{\bar{g} \in \bar{\Gamma}} \sum_{\bar{h} \in \bar{\Gamma}} \operatorname{dim}_{G}(\bar{g} V \cap \bar{h} W) \leq \operatorname{dim}_{G} \ell^{2}(\Gamma) \cdot \operatorname{dim}_{G} V \cdot \operatorname{dim}_{G} W
\end{array}
$$

Proof. The equivalence $\mathcal{I}(V, W) \Leftrightarrow \mathcal{I}_{1}(V, W)$ holds by Lemma 57 .

For the equivalence $\mathcal{I}(V, W) \Leftrightarrow \mathcal{I}_{2}(V, W)$, note that $V \cap \bar{g} W$ is the kernel of the orthogonal projection $\operatorname{pr}\left[\cdot, \bar{g} W^{\perp}\right]: V \rightarrow \bar{g} W^{\perp}$, hence

$$
\operatorname{dim}_{G}(V \cap \bar{g} W)+\operatorname{dim}_{G}\left(p r\left[V, \bar{g} W^{\perp}\right]\right)=k
$$

and

$$
\begin{aligned}
& \sum_{\bar{g} \in \bar{\Gamma}} \operatorname{dim}_{G}(V \cap \bar{g} W) \leq k l \quad \Leftrightarrow \\
& \sum_{\bar{g} \in \bar{\Gamma}}\left[k-\operatorname{dim}_{G}\left(p r\left[V, \bar{g} W^{\perp}\right]\right)\right] \leq k l \quad \Leftrightarrow \\
& \sum_{\bar{g} \in \bar{\Gamma}} \operatorname{dim}_{G}\left(\operatorname{pr}\left[V, \bar{g} W^{\perp}\right]\right) \geq k(n-l) .
\end{aligned}
$$

The rest of the equivalences are similar or easy.

Exercise: write at least 8 other equivalent inequalities.

\section{ERgodicity.}

As before, $G$ is a normal subgroup of finite index in $\Gamma$ and $\bar{\Gamma}:=\Gamma / G$.

\subsection{The definition of ergodicity.}

Definition 59. $A G$-vector in $\ell^{2}(\Gamma)$ is a $G$-subspace $U \in \mathcal{S P}\left(\ell^{2}(\Gamma)\right)^{G}$ with $\operatorname{dim}_{G} U=1$. 
Definition 60. Given a subspace $V \in \mathcal{S P}\left(\ell^{2}(\Gamma)\right)^{G}$, a $G$-vector basis of $V$ is a set $\left\{U_{1}, \ldots, U_{k}\right\}$ of $G$-vectors in $V$ such that the map

$$
\bigoplus_{i=1}^{k} U_{i} \rightarrow \sum_{i=1}^{k} U_{i} \subseteq V
$$

induced by the inclusions $U_{i} \subseteq V$ is a weak isomorphism. If this map is an isometric isomorphism, the $G$-vector basis is called orthonormal.

Definition 61. A $G$-vector $U$ in $\ell^{2}(\Gamma)$ will be called ergodic if the map

$$
\bigoplus_{\bar{g} \in \bar{\Gamma}} \bar{g} U \rightarrow \sum_{\bar{g} \in \bar{\Gamma}} \bar{g} U \subseteq \ell^{2}(\Gamma)
$$

induced by the inclusions $\bar{g} U \subseteq \ell^{2}(\Gamma)$ is injective, or equivalently, is a weak isomorphism.

Definition 62. A subspace $V \in \mathcal{S P}\left(\ell^{2}(\Gamma)\right)^{G}$ will be called ergodic if $V$ and $V^{\perp}$ admit $G$-vector bases that consist of ergodic $G$-vectors.

For a family $\mathcal{F} \subseteq \mathcal{S P}\left(\ell^{2}(\Gamma)\right)^{G}$, let $\mathcal{F}^{\cap}$ denote the closure of $\mathcal{F}$ under intersections.

Definition 63. A family $\mathcal{F} \subseteq \mathcal{S P}\left(\ell^{2}(\Gamma)\right)^{G}$ will be called ergodic if each $V \in \mathcal{F}^{\cap}$ is ergodic.

Theorem 64. If $\left(\mathcal{S}^{\circ}, \mathcal{S}\right)$ is a connected symmetric pair of graph systems and $V$ and $W$ come from $\left(\mathcal{S}^{\circ}, \mathcal{S}\right)$, then the family $\bar{\Gamma} V \cup \bar{\Gamma} W \subseteq \mathcal{S P}\left(\ell^{2}(\Gamma)\right)^{G}$ is ergodic.

Here $\bar{\Gamma} V$ means the set $\{\bar{g} V \mid \bar{g} \in \bar{\Gamma}\}$.

Proof. By the definition in (37),

$$
V=\mathrm{H}(\hat{\alpha}(K)) \quad \text { and } \quad W=\mathrm{H}(\hat{\beta}(L)),
$$

then $V \cap W=\hat{\beta} \circ \hat{\nu}(K \hat{\square} L)$.

Consider the $G$-equivariant map $\hat{\beta} \circ \hat{\nu}: K \hat{\square} L \rightarrow \hat{X}$, or equivalently the $\Gamma$-equivariant map $\hat{\beta} \circ \hat{\nu}: \bar{\Gamma}(K \hat{\square} L) \rightarrow \hat{X} . G$ is the stabilizer of $K^{\circ}, L^{\circ}$, and $K^{\circ} \hat{\square} L^{\circ}$, hence its elements stabilize $K$, $L$, and $K \hat{\square} L$. One can pick an orthonormal $G$-basis $\mathcal{C}$ of $\mathrm{H}(K \hat{\square} L)$ inscribed in $\operatorname{Comp}(K \hat{\square} L)$ by adding one $G$-orbit of edges, or one atom, at a time as in Theorem 30 . This is also an orthonormal $\Gamma$-basis of $\mathrm{H}(\bar{\Gamma}(K \hat{\square} L))$. Then for each $c \in \mathcal{C}, \overline{\operatorname{span}}(\hat{\beta} \circ \hat{\nu}(G c))$ is a $G$-vector. This $G$-vector is ergodic because the map $\hat{\beta} \circ \hat{\nu}$ maps $\overline{\operatorname{span}}(\Gamma c)$ injectively to $\mathrm{H}(\hat{X})=\ell^{2}(\Gamma)$. All such $G$-vectors form a $G$-vector basis of $\mathrm{H}(K \hat{\square} L)$, and their images under $\hat{\beta} \circ \hat{\nu}$ give an orthonormal $G$-vector basis of $V \cap W$. Now continue adding $G$-orbits of edges one by one to go from $K \hat{\square} L$ to $K^{\circ} \hat{\square} L^{\circ}$. This similarly gives an orthonormal $G$-vector basis of $(V \cap W)^{\perp}$. Thus $V \cap W$ is ergodic.

The same argument applies to all intersections $\bar{g} V \cap \bar{h} W$ for $\bar{g} V \in \bar{\Gamma} V$ and $\bar{h} W \in \bar{\Gamma} W$. Furthermore, it generalizes inductively to all finite intersections of elements of the family $\bar{\Gamma} V \cup$ $\bar{\Gamma} W$. Since this family is finite, its closure under intersections is the family of finite intersections.

Theorem 65. If inequality $\mathcal{I}(V, W)$ holds for all pairs $V, W \in \mathcal{S P}\left(\ell^{2}(\Gamma)\right)^{G}$ such that the family $\bar{\Gamma} V \cup \bar{\Gamma} W \subseteq \mathcal{S P}\left(\ell^{2}(\Gamma)\right)^{G}$ is ergodic, then $S H N C$ holds. 
Proof. This follows from theorems 56 and 64.

Theorem 66. If $\left(\mathcal{S}^{\circ}, \mathcal{S}\right)$ is a connected symmetric pair of graph systems or of surface systems, $V$ and $W$ come from $\left(\mathcal{S}^{\circ}, \mathcal{S}\right)$, and $\operatorname{dim}_{G} W=1$, then the following inequality holds.

$$
\mathcal{I}(V, W): \quad \sum_{\bar{g} \in \bar{\Gamma}} \operatorname{dim}_{G}(V \cap \bar{g} W) \leq \operatorname{dim}_{G} V \cdot \operatorname{dim}_{G} W=\operatorname{dim}_{G} V .
$$

Proof. Let $\varphi$ be the composition

$$
\bigoplus_{\bar{g} \in \bar{\Gamma}}(V \cap \bar{g} W) \subseteq \bigoplus_{\bar{g} \in \bar{\Gamma}} \bar{g} W \rightarrow \sum_{\bar{g} \in \bar{\Gamma}} \bar{g} W \subseteq \ell^{2}(\Gamma),
$$

then $\overline{\varphi\left(\bigoplus_{\bar{g} \in \bar{\Gamma}}(V \cap \bar{g} W)\right)} \subseteq V$. Since the $G$-vector $W$ is ergodic (as in Definition 61), $\varphi$ is injective. Then

$$
\begin{aligned}
& \sum_{\bar{g} \in \bar{\Gamma}} \operatorname{dim}_{G}(V \cap \bar{g} W)=\operatorname{dim}_{G} \bigoplus_{\bar{g} \in \bar{\Gamma}}(V \cap \bar{g} W) \\
& =\operatorname{dim}_{G} \overline{\varphi\left(\bigoplus_{\bar{g} \in \bar{\Gamma}}(V \cap \bar{g} W)\right)} \leq \operatorname{dim}_{G} V=\operatorname{dim}_{G} V \cdot \operatorname{dim}_{G} W .
\end{aligned}
$$

Theorems 66 and 56 imply that SHNC holds if $\operatorname{dim}_{G} W=1$. This gives another proof of the result of Tardos [25] and generalizes it to surfaces.

12.2. A test case: finite-group representations. To test the inequality $\mathcal{I}(V, W)$ (which is equivalent to SHNC by Theorem 56), it might be helpful to consider it in the case when $\Gamma$ is finite of order $n$ and $G$ is trivial. Let $\mathbb{C} \Gamma$ be the complex group algebra of $\Gamma$. Then $\operatorname{dim}_{G}$ is the usual dimension dim of vector spaces and $\operatorname{dim} \mathbb{C} \Gamma=|\Gamma|=n$.

Suppose $V$ and $W$ are subspaces of $\mathbb{C} \Gamma$ and $\mathcal{F}^{\cap}$ is the closure of the family

$$
\mathcal{F}:=\{g V \mid g \in \Gamma\} \cup\{h W \mid h \in \Gamma\} \subseteq \mathcal{S P}(\mathbb{C} \Gamma)
$$

under finite intersections. Suppose further that for each $V \in \mathcal{F}^{\cap}$ there is an orthonormal basis $\left\{a_{1}, \ldots, a_{n}\right\}$ over $\mathbb{C}$ such that for some $k \leq n, V=\operatorname{span}\left(a_{1}, \ldots, a_{k}\right)$, and for each $j=1, \ldots, n$, the orbit $\Gamma a_{j}$ is a (not necessarily orthogonal) basis of $\mathbb{C} \Gamma$ over $\mathbb{C}$. Then is it true that

$$
\sum_{g \in \Gamma} \operatorname{dim}(V \cap g W) \leq \operatorname{dim} V \cdot \operatorname{dim} W ?
$$

Are there any conditions that imply this inequality?

12.3. A non-ergodic finite-dimensional example. Take any nontrivial finite group $\Gamma$. Let $\mathbb{C} \Gamma$ be its group algebra and $V=W$ be its vector subspace spanned by the vector

$$
\sum_{g \in \Gamma} g \in \mathbb{C} \Gamma
$$


This subspace is $\Gamma$-invariant. We have

$$
\sum_{g \in \Gamma} \operatorname{dim}(V \cap g W)=\sum_{g \in \Gamma} \operatorname{dim} V=\sum_{g \in \Gamma} 1=|\Gamma|>1=\operatorname{dim} V \cdot \operatorname{dim} W,
$$

which fails inequality $\mathcal{I}(V, W)$. This example suggests that some kind of ergodicity assumption is necessary for SHNC to hold.

12.4. A special case: one edge less than cover. The following theorem proves SHNC in the case when the subgroups $A$ and $B$ are represented by graphs $Y$ and $Z$ that can be embedded in a common finite cover $Z^{\circ}$ of $X$, and $Z$ is one edge less than $Z^{\circ}$. This situation can be viewed as dual to the case when $B$ is of rank 2 (codimension 1 versus dimension 1 ).

Theorem 67. Let $\left(\mathcal{S}^{\circ}, \mathcal{S}\right)$ be a connected symmetric pair of graph systems as in 6.2 such that $Z^{\circ} \backslash Z$ is one edge. Then $S H N C$ holds for $(\Gamma, A, B)$.

Proof. The pair of systems $\left(\mathcal{S}^{\circ}, \mathcal{S}\right)$ gives rise to a $(\Gamma, G)$-arrangement as in 11.3 and to $G$ subspaces $V$ and $W$ of $\ell^{2}(\Gamma)$. Denote

$$
n:=\operatorname{dim}_{G} \ell^{2}(\Gamma)=|\bar{\Gamma}|, \quad k:=\operatorname{dim}_{G} V .
$$

We can assume that the edge $Z^{\circ} \backslash Z$ is essential in $Z^{\circ}$, otherwise $W=\ell^{2}(\Gamma)$ and inequality $\mathcal{I}(V, W)$ is immediate. Then $\operatorname{dim}_{G} W=n-1$. Denote

$$
\begin{aligned}
& \Lambda_{k-1}:=\left\{\bar{g} \in \bar{\Gamma} \mid \operatorname{dim}_{G}(V \cap \bar{g} W)=k-1\right\}, \\
& \Lambda_{k}:=\left\{\bar{g} \in \bar{\Gamma} \mid \operatorname{dim}_{G}(V \cap \bar{g} W)=k\right\}=\{\bar{g} \in \bar{\Gamma} \mid V \subseteq \bar{g} W\}, \\
& \lambda_{k-1}:=\# \Lambda_{k-1}, \quad \lambda_{k}:=\# \Lambda_{k},
\end{aligned}
$$

then $\bar{\Gamma}=\Lambda_{k-1} \sqcup \Lambda_{k}$ and $\lambda_{k-1}=n-\lambda_{k}$. By the ergodicity of $W$, there is a Hilbert subspace $W_{n} \subseteq U$ such that $\ell^{2}(\Gamma)=W \oplus W_{n}$ and $\oplus_{\bar{g} \in \Gamma} \bar{g} W_{n} \rightarrow \sum_{\bar{g} \in \Gamma} \bar{g} W_{n} \subseteq U$ is injective. This implies that $W=\left(W_{n}\right)^{\perp}$ and $\operatorname{dim}_{G}\left(\sum_{\bar{g} \in \Lambda_{k}} \bar{g} W_{n}\right)=\lambda_{k}$. The inclusion

$$
V \subseteq \bigcap_{\bar{g} \in \Lambda_{k}} \bar{g} W=\bigcap_{\bar{g} \in \Lambda_{k}} \bar{g}\left(W_{n}\right)^{\perp}=\bigcap_{\bar{g} \in \Lambda_{k}}\left(\bar{g} W_{n}\right)^{\perp}=\left(\sum_{\bar{g} \in \Lambda_{k}} \bar{g} W_{n}\right)^{\perp}
$$

implies $k \leq n-\lambda_{k}$. The equivalences

$$
\begin{aligned}
& k \leq n-\lambda_{k} \Leftrightarrow \\
& (k-1)\left(n-\lambda_{k}\right)+k \lambda_{k} \leq k(n-1) \Leftrightarrow \\
& (k-1) \lambda_{k-1}+k \lambda_{k} \leq k(n-1) \Leftrightarrow \\
& \sum_{\bar{g} \in \Lambda_{k-1}} \operatorname{dim}_{G}(V \cap \bar{g} W)+\sum_{\bar{g} \in \Lambda_{k}} \operatorname{dim}_{G}(V \cap \bar{g} W) \leq \operatorname{dim}_{G} V \cdot \operatorname{dim}_{G} W \Leftrightarrow \\
& \sum_{\bar{g} \in \bar{\Gamma}} \operatorname{dim}_{G}(V \cap \bar{g} W) \leq \operatorname{dim}_{G} V \cdot \operatorname{dim}_{G} W
\end{aligned}
$$

prove inequality $\mathcal{I}(V, W)$, so SHNC holds for these $(\Gamma, A, B)$ by Theorem 56 . 


\section{Multiplicativity.}

In the case when $\alpha: Y \rightarrow X$ and $\beta: Z \rightarrow X$ are immersions of finite graphs, $S$ is their pull-back, and $\alpha$ or $\beta$ is a cover (equivalently, $[\Gamma: A]<\infty$ or $[\Gamma: B]<\infty$ ), it is well-known that

$$
\bar{r}(S)=\bar{r}(Y) \cdot \bar{r}(Z)
$$

(see [19]). In this section we give an analytic proof of this and discuss when similar equalities hold for dimensions and $\ell^{2}$-Betti numbers.

13.1. A special case: finite sets and finite-dimensional spaces. The following lemma says that the analog of inequality (38) for the counting measures on finite sets is true in the strong sense.

Lemma 68 (Multiplicativity for finite sets). If $\Gamma$ is a finite group and $M$ and $N$ are arbitrary subsets of $\Gamma$, then

$$
\sum_{g \in \Gamma} \#(M \cap g N)=\# M \cdot \# N
$$

Proof.

$$
\begin{aligned}
& \sum_{g \in \Gamma} \#(M \cap g N)=\sum_{g \in \Gamma} \sum_{m \in M} \sum_{n \in N} \#(\{m\} \cap\{g n\}) \\
& =\sum_{m \in M} \sum_{n \in N} \#(\{m\} \cap\{\Gamma n\})=\sum_{m \in M} \sum_{n \in N} 1=\# M \cdot \# N .
\end{aligned}
$$

Lemma 69 (Multiplicativity for standard finite-dimensional spaces). If $\Gamma$ is a finite group, $V$ and $W$ are subspaces of $\mathbb{C} \Gamma$ spanned by some subsets of the standard basis $\{g \cdot 1 \in \mathbb{C} \Gamma \mid g \in \Gamma\}$, then

$$
\sum_{g \in \Gamma} \operatorname{dim}(V \cap g W)=\operatorname{dim}(V) \cdot \operatorname{dim}(W)
$$

Proof. The same proof as for Lemma 68 using the standard basis.

13.2. Multiplicativity for arrangements. Lemma 69 also generalizes word-by-word to the following statement for pairs $(\Gamma, G)$.

Lemma 70 (Multiplicativity for standard $G$-subspaces). If $\Gamma$ is a group, $G$ is a normal subgroup of finite index in $\Gamma, V, W \in \mathcal{S P}\left(\ell^{2}(\Gamma)\right)^{G}$ are subspaces of $\ell^{2}(\Gamma)$ spanned by some subsets of the standard $G$-basis $\left\{\bar{g} \ell^{2}(G) \mid \bar{g} \in \bar{\Gamma}\right\}$ of $\ell^{2}(\Gamma)$, then

$$
\sum_{\bar{g} \in \bar{\Gamma}} \operatorname{dim}_{G}(V \cap \bar{g} W)=\operatorname{dim}_{G}(V) \cdot \operatorname{dim}_{G}(W) .
$$


Lemma 71 (Multiplicativity for $G$-subspaces). Suppose $V, W \in \mathcal{S P}\left(\ell^{2}(\Gamma)\right)^{G}$. If $V=0$ or $W=0$ or $V=\ell^{2}(\Gamma)$ or $W=\ell^{2}(\Gamma)$, then

$$
\sum_{\bar{g} \in \bar{\Gamma}} \operatorname{dim}_{G}(V \cap \bar{g} W)=\operatorname{dim}_{G} V \cdot \operatorname{dim}_{G} W .
$$

Proof. If $V=\ell^{2}(\Gamma)$ then

$$
\sum_{\bar{g} \in \bar{\Gamma}} \operatorname{dim}_{G}(V \cap \bar{g} W)=\sum_{\bar{g} \in \bar{\Gamma}} \operatorname{dim}_{G} \bar{g} W=n \cdot \operatorname{dim}_{G} W=\operatorname{dim}_{G} V \cdot \operatorname{dim}_{G} W .
$$

The rest is clear.

Lemma 72 (Multiplicativity for finite covers). Let $\mathcal{S}$ be a connected graph system as in 3.7 or a connected surface system as in 8.1. If $\alpha: Y \rightarrow X$ or $\beta: Z \rightarrow X$ is a (finite) cover then

$$
b_{1}^{(2)}(\hat{S} ; \Gamma)=b_{1}^{(2)}(\hat{Y} ; \Gamma) \cdot b_{1}^{(2)}(\hat{Z} ; \Gamma) .
$$

In particular, SHNC holds for $\mathcal{S}$.

First proof. The statement follows from Lemma 71 by taking $V$ and $W$ to be the $G$-subspaces coming from the system.

Second proof. If $\beta: Z \rightarrow X$ a cover, take the standard $\Gamma \times \Gamma$-basis $\{e\}$ of $\mathrm{H}(\hat{X}) \cong \ell^{2}(\Gamma \times \Gamma)$. It is possible to lift it to $\hat{Z}$ because $\hat{\beta}: \hat{Z} \rightarrow \hat{X}$ is a cover leafage. The lift is an orthonormal $\Gamma$-basis of $\mathrm{H}(\hat{Z})=\mathrm{H}\left(\hat{Z}^{\circ}\right)$. Check that for each $b$ in the lift, the restricted map $\hat{\beta}: \overline{\operatorname{span}}(\Gamma b) \rightarrow \mathrm{H}(\hat{X})$ is an isometric isomorphism. Then Theorem 32 applies, and, moreover, the proof of Theorem 32 provides the equality $b_{1}^{(2)}(\hat{S} ; \Gamma)=b_{1}^{(2)}(\hat{Y} ; \Gamma) \cdot b_{1}^{(2)}(\hat{Z} ; \Gamma)$ (rather than an inequality).

13.3. Multiplicativity for square maps. Suppose $U \subseteq \ell^{2}(\Gamma \times \Gamma)$ and a subbasis $\mathcal{C}$ come from a symmetric pair of graph or surface systems $\left(\mathcal{S}^{\circ}, \mathcal{S}\right)$ as in 10.7. Then there exists an orthonormal $G \times G$-basis $\mathcal{C}^{\circ}$ of $\ell^{2}(\Gamma \times \Gamma)$ inscribed in $\operatorname{Diag}_{G}^{\Gamma}$ such that $\mathcal{C} \subseteq \mathcal{C}^{\circ}$.

Lemma 73. The following statements are equivalent.

(a) $\nabla_{\mathcal{C}^{\circ}}: \ell^{2}(\Gamma \times \Gamma) \rightarrow \ell^{2}(\Gamma \times \Gamma)$ is injective.

(b) $\nabla_{\mathcal{C}^{\circ}}\left(\ell^{2}(\Gamma \times \Gamma)\right)$ is dense in $\ell^{2}(\Gamma \times \Gamma)$.

$(\mathrm{c}) \nabla_{\mathcal{C}^{\circ}}: \ell^{2}(\Gamma \times \Gamma) \rightarrow \ell^{2}(\Gamma \times \Gamma)$ is a weak isomorphism.

Proof. Since $\operatorname{dim}_{G \times G} \ell^{2}(\Gamma \times \Gamma)<\infty$, the statement follows from the additivity of dimension.

If the following condition holds for a $G \times G$-basis $\mathcal{C}^{\circ}$ coming from $\left(\mathcal{S}^{\circ}, \mathcal{S}\right)$, it would be a correct formulation of multiplicativity (Lemma 72) for the general case when the immersions $\alpha$ and $\beta$ are not assumed to be covers.

Condition 2. The glide square map $\diamond_{\mathcal{C}^{\circ}}: \ell^{2}(\Gamma \times \Gamma) \rightarrow \ell^{2}(\Gamma \times \Gamma)$ is a weak isomorphism.

Lemma 74. If a $G \times G$-basis $\mathcal{C}^{\circ}$ coming from $\left(\mathcal{S}^{\circ}, \mathcal{S}\right)$ satisfies Condition 2 then $S H N C$ holds for $\mathcal{S}$.

Proof. $\nabla_{\mathcal{C}}$ is a restriction of $\nabla_{\mathcal{C}^{\circ}}$, therefore it is injective, so SHNC follows from Theorem 51. 


\section{Questions.}

The notions in this article lead beyond HNC. We ask some questions that arise from the above considerations and seem to be interesting.

Question 1. Suppose $G$ is a finite index subgroup in $\Gamma$ and $U$ is a Hilbert $G \times G$-submodule of $\ell^{2}(\Gamma \times \Gamma)$ that admits an orthonormal $G \times G$-basis inscribed in $\operatorname{Diag}_{G}^{\Gamma}$. Then does there exist a subgroup $H$ of finite index in $\Gamma$ and an $H \times H$-basis $\mathcal{C}$ of $U$ inscribed in $\operatorname{Diag}_{H}^{\Gamma}$ such that the square map $\diamond_{\mathcal{C}}: U \rightarrow \ell^{2}(\Gamma \times \Gamma)$ is injective? Is this true if $U$ comes from a system as in 10.7?

Question 2. Does the free group of rank 2 belong to the class $\mathcal{P}$ in 10.9? Or to $\mathcal{P}_{\text {lim }}$ ? Does the infinite cyclic group belong to $\mathcal{P}$ or to $\mathcal{P}_{\text {lim }}$ ?

Question 3. If $\Gamma$ is the fundamental group of a compact surface of Euler characteristics -1 , then does $\Gamma$ belong to $\mathcal{P}$ or to $\mathcal{P}_{\text {lim }}$ ?

Question 4. Do all finite groups belong to $\mathcal{P}$, or equivalently, to $\mathcal{P}_{\text {lim }}$ ?

Question 5. Are there any groups that do not belong to $\mathcal{P}_{\text {lim }}$ ? Is there a residually finite group that does not belong to $\mathcal{P}_{\text {lim }}$ ?

Question 6. Are atomic square maps injective? (See 10.10.)

The affirmative answer would imply SHNC.

Question 7. Let $\Gamma$ be a group. For which bases of $\ell^{2}(\Gamma \times \Gamma)$ inscribed in $(\Gamma \times \Gamma) / \Delta$ is the corresponding glide square map $\diamond: \ell^{2}(\Gamma \times \Gamma) \rightarrow \ell^{2}(\Gamma \times \Gamma)$ a weak isomorphism, and for which is it not?

Question 8. If $V, W \in \mathcal{S P}\left(\ell^{2}(\Gamma)\right)^{G}$ and the family $\bar{\Gamma} V \cup \bar{\Gamma} W \subseteq \mathcal{S P}\left(\ell^{2}(\Gamma)\right)^{G}$ is ergodic (see 12.1), then does the following inequality hold?

$$
\mathcal{I}(V, W): \quad \sum_{\bar{g} \in \bar{\Gamma}} \operatorname{dim}_{G}(V \cap \bar{g} W) \leq \operatorname{dim}_{G} V \cdot \operatorname{dim}_{G} W .
$$

An affirmative answer would imply SHNC by Theorem 65 .

Question 9. In the case of finite groups, does inequality (38) hold?

\section{REFERENCES}

[1] M. F. АтіYAн, Elliptic operators, discrete groups and von Neumann algebras, in Colloque "Analyse et Topologie" en l'Honneur de Henri Cartan (Orsay, 1974), Soc. Math. France, 1976, pp. 43-72. Astérisque, No. 32-33.

[2] R. G. Burns, On the intersection of finitely generated subgroups of a free group., Math. Z., 119 (1971), pp. $121-130$.

[3] W. Dicks, Equivalence of the strengthened Hanna Neumann conjecture and the amalgamated graph conjecture, Invent. Math., 117 (1994), pp. 373-389.

[4] W. Dicks and E. FormaneK, The rank three case of the Hanna Neumann conjecture, J. Group Theory, 4 (2001), pp. 113-151.

[5] W. Dicks And S. V. Ivanov, On the intersection of free subgroups in free products of groups, Math. Proc. Cambridge Philos. Soc., 144 (2008), pp. 511-534. 
[6] K. Feuerman, The Hanna Neumann Conjecture: A flow detection approach. Ph. D. thesis, University of Illinois at Urbana-Champaign.

[7] S. M. Gersten, Intersections of finitely generated subgroups of free groups and resolutions of graphs, Invent. Math., 71 (1983), pp. 567-591.

[8] A. G. Howson, On the intersection of finitely generated free groups, J. London Math. Soc., 29 (1954), pp. $428-434$.

[9] W. ImRich, On finitely generated subgroups of free groups, Arch. Math. (Basel), 28 (1977), pp. 21-24.

[10] S. V. Ivanov, Intersecting free subgroups in free products of groups, Internat. J. Algebra Comput., 11 (2001), pp. 281-290.

[11] _ - On the Kurosh rank of the intersection of subgroups in free products of groups, Adv. Math., 218 (2008), pp. 465-484.

[12] V. Jones And V. S. Sunder, Introduction to subfactors, vol. 234 of London Mathematical Society Lecture Note Series, Cambridge University Press, Cambridge, 1997.

[13] B. Khan, Positively generated subgroups of free groups and the Hanna Neumann conjecture, in Combinatorial and geometric group theory (New York, 2000/Hoboken, NJ, 2001), vol. 296 of Contemp. Math., Amer. Math. Soc., Providence, RI, 2002, pp. 155-170.

[14] P. A. Linnell, Division rings and group von Neumann algebras, Forum Math., 5 (1993), pp. 561-576.

[15] W. LüCK, $L^{2}$-invariants: theory and applications to geometry and $K$-theory, vol. 44 of Ergebnisse der Mathematik und ihrer Grenzgebiete. 3. Folge. A Series of Modern Surveys in Mathematics [Results in Mathematics and Related Areas. 3rd Series. A Series of Modern Surveys in Mathematics], Springer-Verlag, Berlin, 2002.

[16] J. Meakin And P. Weil, Subgroups of free groups: a contribution to the Hanna Neumann conjecture, in Proceedings of the Conference on Geometric and Combinatorial Group Theory, Part I (Haifa, 2000), vol. 94, 2002, pp. 33-43.

[17] H. Neumann, On the intersection of finitely generated free groups, Publ. Math. Debrecen, 4 (1956), pp. $186-189$.

[18] — On the intersection of finitely generated free groups. Addendum, Publ. Math. Debrecen, 5 (1957), p. 128.

[19] W. D. Neumann, On intersections of finitely generated subgroups of free groups, in Groups-Canberra 1989, vol. 1456 of Lecture Notes in Math., Springer, Berlin, 1990, pp. 161-170.

[20] P. Nickolas, Intersections of finitely generated free groups, Bull. Austral. Math. Soc., 31 (1985), pp. 339348.

[21] P. ScotT, Subgroups of surface groups are almost geometric, J. London Math. Soc. (2), 17 (1978), pp. 555565.

[22] — Correction to: "Subgroups of surface groups are almost geometric" [J. London Math. Soc. (2) 17 (1978), no. 3, 555-565; MR0494062 (58 \#12996)], J. London Math. Soc. (2), 32 (1985), pp. 217-220.

[23] B. Servatius, A short proof of a theorem of Burns, Math. Z., 184 (1983), pp. 133-137.

[24] J. R. Stallings, Topology of finite graphs, Invent. Math., 71 (1983), pp. 551-565.

[25] G. TARDos, On the intersection of subgroups of a free group, Invent. Math., 108 (1992), pp. 29-36.

Igor Mineyev, University of Illinois at Urbana-Champaign

mineyev@math.uiuc. edu

http://www.math.uiuc.edu/ ^mineyev/math/ 